\title{
Nonrotating black hole in a post-Newtonian tidal environment II
}

\author{
Eric Poisson and Eamonn Corrigan \\ Department of Physics, University of Guelph, Guelph, Ontario, N1G 2W1, Canada
}

(Dated: May 30, 2018)

\begin{abstract}
In the first part of the paper we construct the metric of a tidally deformed, nonrotating black hole. The metric is presented as an expansion in powers of $r / b \ll 1$, in which $r$ is the distance to the black hole and $b$ the characteristic length scale of the tidal field - the typical distance to the remote bodies responsible for the tidal environment. The metric is expanded through order $(r / b)^{4}$ and written in terms of a number of tidal multipole moments, the gravitoelectric moments $\mathcal{E}_{a b}, \mathcal{E}_{a b c}$, $\mathcal{E}_{a b c d}$ and the gravitomagnetic moments $\mathcal{B}_{a b}, \mathcal{B}_{a b c}, \mathcal{B}_{a b c d}$. It differs from the similar construction of Poisson and Vlasov in that the tidal perturbation is presented in Regge-Wheeler gauge instead of the light-cone gauge employed previously. In the second part of the paper we determine the tidal moments by matching the black-hole metric to a post-Newtonian metric that describes a system of bodies with weak mutual gravity. This extends the previous work of Taylor and Poisson (paper I in this sequence), which computed only the leading-order tidal moments, $\mathcal{E}_{a b}$ and $\mathcal{B}_{a b}$. The matching is greatly facilitated by the Regge-Wheeler form of the black-hole metric, and this motivates the work carried out in the first part of the paper. The tidal moments are calculated accurately through the first post-Newtonian approximation, and at this order they are independent of the precise nature of the compact body. The moments therefore apply equally well to a rotating black hole, or to a (rotating or nonrotating) neutron star. As an application of this formalism, we examine the intrinsic geometry of a tidally deformed event horizon, and describe it in terms of a deformation function that represents a quadrupolar and octupolar tidal bulge.
\end{abstract}

\section{INTRODUCTION AND SUMMARY}

The tidal interaction between neutron stars in a close binary system has recently been the subject of intense investigation, following the observation [1] that the tidal deformation of each body could have a measurable impact on the emitted gravitational waves. The effect depends on the tidal deformability of each neutron star, and a large effort has been deployed to the computation of this quantity for realistic models of neutron stars, and to ascertain the importance of the tidal deformation on the gravitational-wave signal $2[24$. The recent observation of GW170817 by the LIGO and Virgo instruments [25, with its first attempt to measure the tidal deformability of neutron stars, inaugurated a new era of gravitational-wave astronomy that is likely, in the fullness of time, to reveal some aspects of nuclear matter equation of state and neutron-star internal structure.

Detailed modeling of the tidal dynamics of compact objects in general relativity requires the precise specification of the tidal environment in which the compact object resides. In a context in which the tidal field is weak and varies slowly compared with the dynamical timescale of the compact body, the tidal environment can be described in terms of a number of tidal multipole moments. These come in two guises. The gravitoelectric moments $\mathcal{E}_{a b}, \mathcal{E}_{a b c}, \mathcal{E}_{a b c d}$, and so on, are produced by mass densities external to the compact body, and have direct analogues in Newtonian gravity. The gravitomagnetic moments $\mathcal{B}_{a b}, \mathcal{B}_{a b c}, \mathcal{B}_{a b c d}$, and so on, are produced by external mass currents, and have no analogues in Newtonian gravity. A specification of the tidal environment amounts to a determination of the tidal moments in terms of the state of motion of the two-body system. This task is the central concern of this paper.

A method to determine tidal moments, in a context in which the compact object is a member of a post-Newtonian binary system, was developed by Taylor and Poisson in Ref. 26, the first paper in this sequence - hereafter referred to as Paper I. The method, a generalization to compact objects of a previous implementation limited to weakly selfgravitating bodies [27-29, relies on the matching of two distinct metrics in an overlapping domain of validity. The situation is illustrated in Fig. 1 .

The first metric is that of a tidally deformed compact object, presented as a perturbed version of the Schwarzschild metric, and expressed in terms of the tidal multipole moments. This metric is valid in a small domain that surrounds the compact body, and the tidal moments appear in it as freely specifiable functions of time. These cannot be determined by integrating the Einstein field equations in the small domain, because the companion body is excluded and the integration cannot incorporate its precise influence on the gravitational field of the compact object.

The second metric is a post-Newtonian metric given in a large domain that includes both bodies, but leaves out a small region around the compact object, in which gravity is too strong to be adequately captured by a post-Newtonian approximation. In this way, the internal gravity of the compact object is allowed to be strong, while the mutual gravity between bodies is assumed to be weak. Because the domain excludes the compact body, the post-Newtonian metric also contains freely specifiable functions of time.

There exists an overlap between the small domain of the first metric and the large domain of the second metric. 


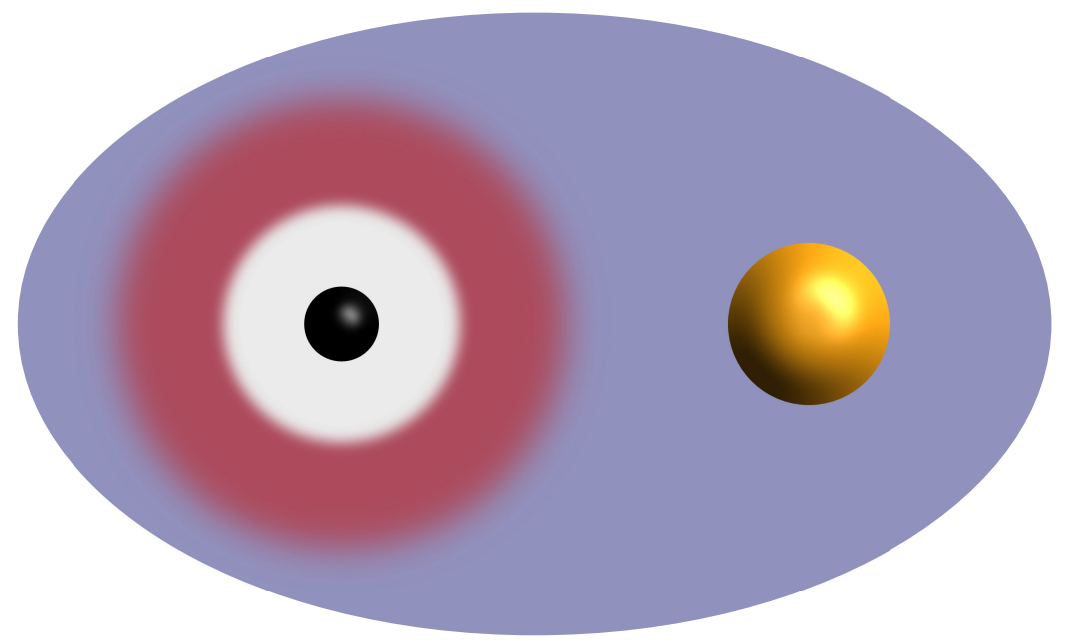

FIG. 1. A post-Newtonian system consisting of a black hole (left, black) and a normal star (right, yellow online). The postNewtonian domain is pictured as an ellipse (blue online), and it excludes the fuzzy white region surrounding the black hole. The black-hole domain is pictured as the dark fuzzy region (red online), which extends all the way down to the black hole. The matching of the black-hole and post-Newtonian metrics is carried out in the overlap between the black-hole and post-Newtonian domains.

Matching the metrics in this overlap determines the tidal moments and the missing details of the post-Newtonian metric. In this manner, the tidal environment of the compact body is determined, up to a desired number of moments, and up to a desired post-Newtonian order. In Paper I [26] the method was exploited to calculate the leading order tidal moments, $\mathcal{E}_{a b}$ and $\mathcal{B}_{a b}$, through the first post-Newtonian (1PN) approximation. In this paper we extend this calculation to the next two tidal moments, $\mathcal{E}_{a b c}$ and $\mathcal{E}_{a b c d}$, as well as $\mathcal{B}_{a b c}$ and $\mathcal{B}_{a b c d}$; this calculation also is carried out to $1 \mathrm{PN}$ order.

The multipole moments are defined precisely in Sec. II] and their scaling with the mass $M_{2}$ of the companion body, the interbody distance $b$, and the orbital velocity $V$ is also described in this section. For concreteness we choose the compact object to be a nonrotating black hole of mass $M_{1}$. The precise nature of the body, however, is of no consequence in the determination of the tidal moments at 1PN order: the effects of spin enter at 1.5PN order, and finite-size effects enter at 5PN order. Our moments, therefore, determine the tidal environment of a neutron star just as well as that of a black hole, and these objects are allowed to rotate.

The construction of the metric of a tidally deformed black hole begins in Sec. III with the introduction of tidal potentials constructed from the tidal moments. The metric is next obtained in $(v, r, \theta, \phi)$ coordinates in Sec. IV. with $v$ denoting advanced time, and transformed to $(t, r, \theta, \phi)$ coordinates in Sec. VV The goal is to obtain a metric accurate through fourth order in an expansion in powers of $r / b$, where $r$ is the distance to the black hole and $b$ is the interbody distance. This metric incorporates terms that involve the tidal quadrupole moments $\mathcal{E}_{a b}$ and $\mathcal{B}_{a b}$ and their time derivatives, the octupole moments $\mathcal{E}_{a b c}$ and $\mathcal{B}_{a b c}$ and their time derivatives, and the hexadecapole moments $\mathcal{E}_{a b c d}$ and $\mathcal{B}_{a b c d}$. The nonlinearity of the field equation implies that terms at order $(r / b)^{4}$ also include bilinear combinations of $\mathcal{E}_{a b}$ and $\mathcal{B}_{a b}$. To achieve all this we rely heavily on the formalism of Poisson and Vlasov [30, which provides an essential foundation for this work.

In fact, the metric of a tidally deformed black hole, accurate through order $(r / b)^{4}$, was already constructed by Poisson and Vlasov, and in principle, this metric could have been imported directly without having to perform the additional work described in Secs. [V and $\mathrm{I}$ The need for this work comes from the fact that the Poisson-Vlasov metric is given in a form that does not facilitate a matching with the post-Newtonian metric. First, the metric is expressed in light-cone coordinates, and its post-Newtonian expansion does not reduce to the standard post-Newtonian form that is required for matching. Second, the Poisson-Vlasov metric is expressed in terms of tidal moments that were specifically defined to simplify the description of the deformed event horizon; these definitions make the postNewtonian expression of the metric more complicated than it has to be.

In our developments in Secs. IV and $\mathrm{V}$, we endeavor to arrive at a form for the black-hole metric that will simplify, to the fullest extent possible, the task of matching it to a post-Newtonian metric. And we aim to achieve this not just at the 1PN order of the calculations carried out in this paper, but also at higher post-Newtonian orders, in preparation for future work. In this regard, the most important property of the black-hole metric is that it becomes compatible with the standard form of the post-Newtonian metric after expansion through 1PN order. This is achieved 
with two technical devices. First, the metric of the unperturbed black hole, given by the Schwarzschild solution, is presented in the harmonic radial coordinate $\bar{r}$, related to the usual areal radius $r$ by $\bar{r}=r-M$. This ensures that the unperturbed metric reduces to the standard post-Newtonian metric after expansion. Second, the tidal perturbation is presented in Regge-Wheeler gauge, which happily produces a perturbed metric that continues to respect the standard post-Newtonian form. Such simplicity could not be achieved with the Poisson-Vlasov metric. It also was not achieved in Paper I [26], with a perturbed metric presented initially in the light-cone gauge. Matching with the post-Newtonian metric required a transformation of the black-hole metric to harmonic coordinates, a technically demanding step that was (in retrospect) unnecessary. Our new construction allows us to avoid this step altogether, and provides a solid infrastructure that will facilitate future extensions of this work.

As stated previously, the metric of a tidally deformed black hole can be expressed in terms of tidal multipole moments that appear as freely specifiable functions of time. The moments, however, are not uniquely defined, and they admit redefinitions that leave the form of the metric unchanged up to integration constants. It was this freedom that was exploited by Poisson and Vlasov [30] to simplify the description of the perturbed horizon. In this work we exploit it differently, to simplify the post-Newtonian expansion of the metric. The redefinition of tidal moments is explored in Sec. VI] and sets of tidal moments that belong to different "calibrations" are related to one another.

The task of matching the black-hole and post-Newtonian metrics begins in Sec. VII with the post-Newtonian expansion of the black-hole metric and the extraction of the gravitational potentials (a Newtonian potential $U$, a vector potential $U_{a}$, and a post-Newtonian potential $\Psi$ ). The matching itself is carried out in Sec. VIII. The final products are the tidal moments expressed in terms of external potentials that represent the gravitational field of the companion body. These are evaluated concretely in Sec. IX, and the tidal moments are finally obtained in terms of the orbital degrees of freedom of the two-body system. At this stage our task is complete: the quadrupole, octupole, and hexadecapole tidal moments are all determined through the first post-Newtonian order. We note that the octupole tidal moments were previously given in Ref. 31 for the specific case of circular orbits; our expressions agree with theirs. The hexadecapole moments are presented here for the first time.

As an application of our results, in Sec. $\mathrm{X}$ we examine the intrinsic geometry of the tidally deformed event horizon. To describe the deformation it is useful to introduce a fictitious two-dimensional surface embedded in a threedimensional flat space, to describe this surface by the equation $r=2 M_{1}(1+\varepsilon)$, and to choose the displacement function $\varepsilon$ in such a way that the embedded surface possesses the same intrinsic geometry as a two-dimensional cross-section of the event horizon. In relativistic units in which $G=c=1$, used throughout the paper, we find that $\varepsilon$ is given by

$$
\begin{aligned}
\varepsilon= & \frac{1}{2} q_{1}^{2} q_{2}\left(\frac{M}{b}\right)^{3}\left\{-\left[1+\frac{1}{2} q_{1} V^{2}\right]\left(3 \cos ^{2} \theta-1\right)+3\left[1+\frac{1}{2}\left(q_{1}-4\right) V^{2}\right] \sin ^{2} \theta \cos (2 \psi)\right. \\
& \left.-\frac{3}{5} q_{1} V^{2} \sin \theta\left(5 \cos ^{2} \theta-1\right) \cos (\psi)+q_{1} V^{2} \sin ^{3} \theta \cos (3 \psi)+1.5 \mathrm{PN}\right\},
\end{aligned}
$$

where $M:=M_{1}+M_{2}, q_{1}:=M_{1} / M, q_{2}:=M_{2} / M, V=(M / b)^{1 / 2}$ is the orbital velocity, and $(\theta, \phi)$ are polar angles that specify a position on the embedded surface, with the polar axis taken to be normal to the orbital plane. The phase function is defined by $\psi:=\phi-\bar{\omega} v$, with $v$ representing advanced-time on the horizon, and

$$
\bar{\omega}=\sqrt{\frac{M}{b^{3}}}\left[1-\frac{1}{2}\left(3+q_{1} q_{2}\right) V^{2}+2 \mathrm{PN}\right] .
$$

is the angular frequency of the tidal field.

The displacement function can be decomposed into a quadrupole term that is independent of the phase $\psi$, and another quadrupole term that oscillates at twice the frequency $\bar{\omega}$; both terms originate from $\mathcal{E}_{a b}$. The remaining terms at $1 \mathrm{PN}$ order consist of octupole deformations that oscillate at once and three times the tidal frequency; both terms originate from $\mathcal{E}_{a b c}$, and they were omitted in the earlier statement of this result given in Paper I. The hexadecapole tidal moment $\mathcal{E}_{a b c d}$ makes no appearance in $\varepsilon$ at 1PN order, and the gravitomagnetic moments are excluded on the grounds that they have the wrong parity to contribute to a scalar quantity such as $\varepsilon$. The tidal displacement represents a bulge aligned with $\phi=\bar{\omega} v$.

Some of the calculations presented in the main text rely on tables and technical developments relegated to three appendices. In Appendix A we provide decompositions of Cartesian tensors into irreducible pieces, and in Appendix B we record various derivatives of a distance function that appears in gravitational potentials. In Appendix $\mathrm{C}$ we display a large number of tables that contain definitions of various quantities used in our calculations. 


\section{TIDAL MOMENTS AND SCALES}

We consider a nonrotating black hole of mass $M_{1}$ immersed in a tidal environment described by the gravitoelectric tidal moments $\mathcal{E}_{a b}, \mathcal{E}_{a b c}, \mathcal{E}_{a b c d}$, and the gravitomagnetic tidal moments $\mathcal{B}_{a b}, \mathcal{B}_{a b c}, \mathcal{B}_{a b c d}$; all tidal moments are defined as three-dimensional Cartesian tensors, and are functions of time only. The gravitational field of the tidally deformed black hole is described by a metric $g_{\alpha \beta}$ presented as an expansion in powers of $r / b \ll 1$, where $r$ is the distance to the black hole and $b$ is the characteristic length scale of the tidal field - the typical distance to the remote bodies responsible for the tidal environment. The tidal moments determine the behavior of the metric when $r \gg M_{1}$, and their time dependence is arbitrary; it cannot be determined by integrating the field equations locally, in a neighborhood of the black hole limited to $r \ll b$. To determine the tidal moments, the local metric $g_{\alpha \beta}$ must be matched to a global metric that extends beyond $r=b$ and includes the remote bodies.

The tidal moments can be loosely interpreted as describing the Weyl curvature of the tidally deformed black hole in a distance interval $M_{1} \ll r \ll b$, in which the tidal field dominates over the hole's own gravity. Formally, however, the tidal moments are defined in terms of the Weyl tensor of a different, but related, spacetime. The metric of this spacetime is the limit of $g_{\alpha \beta}$ when $M_{1}$ is taken to zero while keeping the tidal moments fixed. The Weyl tensor and its covariant derivatives are then computed for this metric, and their components in local Lorentzian coordinates $\left(x^{0}, x^{a}\right)$ are evaluated at $r=0$, now describing a regular world line of the spacetime. Additional details regarding the definition of the tidal moments are provided in Sec. II of Ref. [30].

The tidal quadrupole moments are defined by

$$
\begin{aligned}
\mathcal{E}_{a b} & :=\left(C_{0 a 0 b}\right)^{\mathrm{STF}}, \\
\mathcal{B}_{a b} & :=\frac{1}{2}\left(\epsilon_{a p q} C_{b 0}^{p q}\right)^{\mathrm{STF}}
\end{aligned}
$$

in terms of the Weyl tensor $C_{\alpha \beta \gamma \delta}$ of the related spacetime. Here $\epsilon_{a b c}$ is the permutation symbol, and the STF sign instructs us to symmetrize all free indices and remove all traces. This operation is also indicated in an angularbracket notation, such as $A_{\langle a b c d\rangle}:=\left(A_{a b c d}\right)^{\mathrm{STF}}$. The tidal moments are functions of coordinate time $x^{0}$ (either $t$ or advanced-time $v$ ), and we use overdots to indicate differentiation with respect to this coordinate.

The tidal octupole moments are defined by

$$
\begin{aligned}
\mathcal{E}_{a b c} & :=\left(C_{0 a 0 b \mid c}\right)^{\mathrm{STF}} \\
\mathcal{B}_{a b c} & :=\frac{3}{8}\left(\epsilon_{a p q} C_{b 0 \mid c}^{p q}\right)^{\mathrm{STF}},
\end{aligned}
$$

in which a vertical stroke indicates covariant differentiation. These also are functions of coordinate time, and time derivatives are again indicated with overdots.

The tidal hexadecapole moments are defined by

$$
\begin{aligned}
\mathcal{E}_{a b c d} & :=\frac{1}{2}\left(C_{0 a 0 b \mid c d}\right)^{\mathrm{STF}}, \\
\mathcal{B}_{a b c d} & :=\frac{3}{20}\left(\epsilon_{a p q} C_{b 0 \mid c d}^{p q}\right)^{\mathrm{STF}} .
\end{aligned}
$$

The numerical factors inserted in these equations are inherited from Zhang's choice of normalization for the tidal moments 32 .

The tidal environment is characterized by an external mass scale $M_{2}$, a distance scale $b$, a velocity scale $V \sim \sqrt{M / b}$, where $M:=M_{1}+M_{2}$, and an angular-velocity scale $\omega \sim V / b$. The gravitoelectric moments scale as

$$
\mathcal{E}_{a b} \sim \frac{M_{2}}{b^{3}}, \quad \mathcal{E}_{a b c} \sim \frac{M_{2}}{b^{4}}, \quad \mathcal{E}_{a b c d} \sim \frac{M_{2}}{b^{5}}
$$

and the gravitomagnetic moments scale as

$$
\mathcal{B}_{a b} \sim \frac{M_{2} V}{b^{3}}, \quad \mathcal{B}_{a b c} \sim \frac{M_{2} V}{b^{4}}, \quad \mathcal{B}_{a b c d} \sim \frac{M_{2} V}{b^{5}}
$$

Time derivatives of the gravitoelectric tidal moments scale as

$$
\dot{\mathcal{E}}_{a b} \sim \frac{M_{2} V}{b^{4}}, \quad \ddot{\mathcal{E}}_{a b} \sim \frac{M_{2} V^{2}}{b^{5}}, \quad \dot{\mathcal{E}}_{a b c} \sim \frac{M_{2} V}{b^{5}},
$$


while the derivatives of the gravitomagnetic moments scale as

$$
\dot{\mathcal{B}}_{a b} \sim \frac{M_{2} V^{2}}{b^{4}}, \quad \ddot{\mathcal{B}}_{a b} \sim \frac{M_{2} V^{3}}{b^{5}}, \quad \dot{\mathcal{B}}_{a b c} \sim \frac{M_{2} V^{2}}{b^{5}} .
$$

We assume that $b \gg M_{1}$, so that the source of the tidal field is situated far from the black hole. This implies that $V^{2} \ll\left(1+M_{2} / M_{1}\right)$, so that the orbital velocity is much smaller than the speed of light. It also follows that

$$
M_{1} \omega \sim\left(1+M_{2} / M_{1}\right)^{1 / 2}\left(M_{1} / b\right)^{3 / 2} \ll 1,
$$

so that the time scale $\omega^{-1}$ associated with variations of the tidal field is much longer than $M_{1}$, the time scale associated with the black hole. The tidal field is thus assumed to be weak and to vary slowly.

The metric of a tidally deformed black hole shall be presented as an expansion in powers of $r / b$, assuming that $r$, the distance to the black hole, is much smaller than $b$, the distance scale of the tidal field. The metric shall be expanded through order $(r / b)^{4}$, and expressed in terms of tidal potentials constructed from the tidal moments.

\section{TIDAL POTENTIALS}

The metric of a tidally deformed black hole can be expressed in terms of a number of tidal potentials that are constructed from the tidal moments. Details are provided in Sec. II of Ref. [30, and we summarize the main points here.

The tidal moments are combined with $\Omega^{a}:=x^{a} / r$, with $r$ denoting the usual Euclidean distance, so as to form scalar and vector potentials that form an irreducible representation of the rotation group labeled by multipole order $\ell$. (Tensor potentials are not required, by virtue of the gauge choice adopted in Secs. [IV]and $/ \mathrm{V}$ ) Each vector potential is required to be purely transverse, in the sense of being orthogonal to $\Omega^{a}$. The required potentials are displayed in Table 【.

A transformation from Cartesian coordinates $x^{a}$ to spherical coordinates $(r, \theta, \phi)$ is effected by $x^{a}=r \Omega^{a}\left(\theta^{A}\right)$, in which $\Omega^{a}$ is now parameterized by two polar angles $\theta^{A}=(\theta, \phi)$. Explicitly, we have that $\Omega^{a}=[\sin \theta \cos \phi, \sin \theta \sin \phi, \cos \theta]$. The Jacobian matrix is given by

$$
\frac{\partial x^{a}}{\partial r}=\Omega^{a}, \quad \frac{\partial x^{a}}{\partial \theta^{A}}=r \Omega_{A}^{a},
$$

with $\Omega_{A}^{a}:=\partial \Omega^{a} / \partial \theta^{A}$. We have that $\Omega_{A B}:=\delta_{a b} \Omega_{A}^{a} \Omega_{B}^{b}=\operatorname{diag}\left[1, \sin ^{2} \theta\right]$ is the metric on the unit two-sphere, and $\Omega^{A B}$ is its inverse. The inverse of the Jacobian matrix is

$$
\frac{\partial r}{\partial x^{a}}=\Omega_{a}, \quad \frac{\partial \theta^{A}}{\partial x^{a}}=\frac{1}{r} \Omega_{a}^{A}
$$

where $\Omega_{a}^{A}:=\Omega^{A B} \delta_{a b} \Omega_{B}^{b}$.

We introduce $D_{A}$ as the covariant-derivative operator compatible with $\Omega_{A B}$, and $\epsilon_{A B}$ as the Levi-Civita tensor on the unit two-sphere (with nonvanishing components $\epsilon_{\theta \phi}=-\epsilon_{\phi \theta}=\sin \theta$ ). We adopt the convention that uppercase Latin indices are raised and lowered with $\Omega^{A B}$ and $\Omega_{A B}$, respectively. Finally, we note that $D_{C} \Omega_{A B}=D_{C} \epsilon_{A B}=0$.

We convert the vector potentials from their initial Cartesian forms to angular-coordinate versions by making use of the matrix $\Omega_{A}^{a}$. We thus define

$$
\mathcal{B}_{A}^{\mathrm{q}}:=\mathcal{B}_{a}^{\mathrm{q}} \Omega_{A}^{a},
$$

and apply the same rule to all other vector potentials. After this conversion the tidal potentials become scalar and vector fields on the unit two-sphere, and they become independent of $r$. It is easy to show that the conversion is reversed with $\mathcal{B}_{a}^{\mathrm{q}}=\mathcal{B}_{A}^{\mathrm{q}} \Omega_{a}^{A}$.

The tidal potentials can all be expressed in terms of spherical harmonics. Let $Y^{\ell m}$ be real-valued spherical-harmonic functions (as defined in Table II). The relevant vectorial and tensorial harmonics of even parity are

$$
\begin{aligned}
Y_{A}^{\ell m} & :=D_{A} Y^{\ell m}, \\
Y_{A B}^{\ell m} & :=\left[D_{A} D_{B}+\frac{1}{2} \ell(\ell+1) \Omega_{A B}\right] Y^{\ell m} ;
\end{aligned}
$$

notice that $Y_{A B}^{\ell m}$ is tracefree, $\Omega^{A B} Y_{A B}^{\ell m}=0$, by virtue of the eigenvalue equation satisfied by the spherical harmonics. The vectorial and tensorial harmonics of odd parity are

$$
X_{A}^{\ell m}:=-\epsilon_{A}^{B} D_{B} Y^{\ell m}
$$




$$
X_{A B}^{\ell m}:=-\frac{1}{2}\left(\epsilon_{A}^{C} D_{B}+\epsilon_{B}^{C} D_{A}\right) D_{C} Y^{\ell m}=0
$$

the tensorial harmonics $X_{A B}^{\ell m}$ also are tracefree: $\Omega^{A B} X_{A B}^{\ell m}=0$. The decomposition of the tidal potentials in spherical harmonics is presented in Tables III IV, V, VI, VII, and VIII

\section{METRIC IN $(v, r, \theta, \phi)$ COORDINATES}

In this section and the next we construct the metric of a tidally deformed black hole, expanded through order $(r / b)^{4}$. In these sections and the ones that follow, we denote the mass of the black hole by $M$ instead of $M_{1}$. The original practice will be resumed in Sec. IX.

In the standard $\left(v, r, \theta^{A}\right)$ coordinates, the metric of an unperturbed black hole is given by the Schwarzschild solution, which has the nonvanishing components

$$
g_{v v}=-f, \quad g_{v r}=1, \quad g_{A B}=r^{2} \Omega_{A B},
$$

where $f:=1-2 M / r$. The tidal perturbation introduces additional terms in the metric. These are constructed by incorporating a tidal potential such as $\mathcal{E}^{q}\left(v, \theta^{A}\right)$, multiplied by a radial function such as $e_{v v}^{q}(r)$, in the metric. All

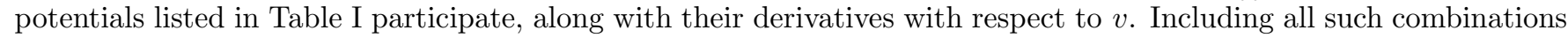
produces a complete metric ansatz that can then be inserted within the Einstein field equations to determine the radial functions.

The perturbation is presented in the Regge-Wheeler gauge (see, for example, Ref. 33 , for definitions and a review of black-hole perturbation theory), which requires even-parity terms to be confined to the $v v, v r, r r$, and $A B$ components of the metric, and odd-parity terms to be confined to the $v A$ and $r A$ components of the metric. In addition, the Regge-Wheeler gauge requires $g_{A B}$ to be proportional to $\Omega_{A B}$. The gauge is uniquely defined for $\ell \geq 2$, but it is not defined when $\ell=0$ and $\ell=1$. The choices made in the monopole and dipole cases will be described below.

The metric of a tidally deformed, nonrotating black hole takes the form of

$$
\begin{aligned}
& g_{v v}=-f+r^{2} e_{v v}^{\mathrm{q}} \mathcal{E}^{\mathrm{a}}+r^{3} \dot{e}_{v v}^{\mathrm{q}} \dot{\mathcal{E}}^{\mathrm{q}}+r^{4} \ddot{e}_{v v}^{\mathrm{q}} \ddot{\mathcal{E}}^{\mathrm{a}}+r^{3} e_{v v}^{\circ} \mathcal{E}^{\circ}+r^{4} \dot{e}_{v v}^{\circ} \dot{\mathcal{E}}^{\circ}+r^{4} e_{v v}^{\mathrm{h}} \mathcal{E}^{\mathrm{h}}+r^{4}\left(p_{v v}^{\mathrm{m}} \mathcal{P}^{\mathrm{m}}+q_{v v}^{\mathrm{m}} \mathcal{Q}^{\mathrm{m}}\right) \\
& +r^{4}\left(p_{v v}^{\mathrm{q}} \mathcal{P}^{\mathrm{a}}+q_{v v}^{\mathrm{a}} \mathcal{Q}^{\mathrm{q}}\right)+r^{4}\left(p_{v v}^{\mathrm{h}} \mathcal{P}^{\mathrm{h}}+q_{v v}^{\mathrm{h}} \mathcal{Q}^{\mathrm{h}}\right)+r^{4}\left(g_{v v}^{\mathrm{d}} \mathcal{G}^{\mathrm{d}}+g_{v v}^{\circ} \mathcal{G}^{\circ}\right)+O\left(r^{5}\right), \\
& g_{v r}=1+r^{2} e_{v r}^{\mathrm{q}} \mathcal{E}^{\mathrm{q}}+r^{3} \dot{e}_{v r}^{\mathrm{q}} \dot{\mathcal{E}}^{\mathrm{q}}+r^{4} \ddot{e}_{v r}^{\mathrm{q}} \ddot{\mathcal{E}}^{\mathrm{q}}+r^{3} e_{v r}^{\circ} \mathcal{E}^{\circ}+r^{4} \dot{e}_{v r}^{\circ} \dot{\mathcal{E}}^{\circ}+r^{4} e_{v r}^{\mathrm{h}} \mathcal{E}^{\mathrm{h}}+r^{4}\left(p_{v r}^{\mathrm{m}} \mathcal{P}^{\mathrm{m}}+q_{v r}^{\mathrm{m}} \mathcal{Q}^{\mathrm{m}}\right) \\
& +r^{4}\left(p_{v r}^{\mathrm{q}} \mathcal{P}^{\mathrm{a}}+q_{v r}^{\mathrm{a}} \mathcal{Q}^{\mathrm{a}}\right)+r^{4}\left(p_{v r}^{\mathrm{h}} \mathcal{P}^{\mathrm{h}}+q_{v r}^{\mathrm{h}} \mathcal{Q}^{\mathrm{h}}\right)+r^{4}\left(g_{v r}^{\mathrm{d}} \mathcal{G}^{\mathrm{d}}+g_{v r}^{\circ} \mathcal{G}^{\circ}\right)+O\left(r^{5}\right) \\
& g_{r r}=r^{2} e_{r r}^{\mathrm{q}} \mathcal{E}^{\mathrm{q}}+r^{3} \dot{e}_{r r}^{\mathrm{q}} \dot{\mathcal{E}}^{\mathrm{q}}+r^{4} \ddot{e}_{r r}^{\mathrm{q}} \ddot{\mathcal{E}}^{\mathrm{q}}+r^{3} e_{r r}^{\circ} \mathcal{E}^{\circ}+r^{4} \dot{e}_{r r}^{\circ} \dot{\mathcal{E}}^{\circ}+r^{4} e_{r r}^{\mathrm{h}} \mathcal{E}^{\mathrm{h}}+r^{4}\left(p_{r r}^{\mathrm{m}} \mathcal{P}^{\mathrm{m}}+q_{r r}^{\mathrm{m}} \mathcal{Q}^{\mathrm{m}}\right) \\
& +r^{4}\left(p_{r r}^{\mathrm{q}} \mathcal{P}^{\mathrm{a}}+q_{r r}^{\mathrm{q}} \mathcal{Q}^{\mathrm{a}}\right)+r^{4}\left(p_{r r}^{\mathrm{h}} \mathcal{P}^{\mathrm{h}}+q_{r r}^{\mathrm{h}} \mathcal{Q}^{\mathrm{h}}\right)+r^{4}\left(g_{r r}^{\mathrm{d}} \mathcal{G}^{\mathrm{d}}+g_{r r}^{\circ} \mathcal{G}^{\circ}\right)+O\left(r^{5}\right), \\
& g_{v A}=r^{3} b_{v}^{\mathrm{q}} \mathcal{B}_{A}^{\mathrm{q}}+r^{4} \dot{b}_{v}^{\mathrm{q}} \dot{\mathcal{B}}_{A}^{\mathrm{q}}+r^{5} \ddot{b}_{v}^{\mathrm{q}} \ddot{\mathcal{B}}_{A}^{\mathrm{q}}+r^{4} b_{v}^{\circ} \mathcal{B}_{A}^{\circ}+r^{5} \dot{b}_{v}^{\circ} \dot{\mathcal{B}}_{A}^{\circ}+r^{5} b_{v}^{\mathrm{h}} \mathcal{B}_{A}^{\mathrm{h}}+r^{5}\left(h_{v}^{\mathrm{q}} \mathcal{H}_{A}^{\mathrm{q}}+h_{v}^{\mathrm{h}} \mathcal{H}_{A}^{\mathrm{h}}\right)+O\left(r^{6}\right) \text {, } \\
& g_{r A}=r^{3} b_{r}^{\mathrm{q}} \mathcal{B}_{A}^{\mathrm{q}}+r^{4} \dot{b}_{r}^{\mathrm{q}} \dot{\mathcal{B}}_{A}^{\mathrm{q}}+r^{5} \ddot{b}_{r}^{\mathrm{q}} \ddot{\mathcal{B}}_{A}^{\mathrm{q}}+r^{4} b_{r}^{\circ} \mathcal{B}_{A}^{\circ}+r^{5} \dot{b}_{r}^{\circ} \dot{\mathcal{B}}_{A}^{\circ}+r^{5} b_{r}^{\mathrm{h}} \mathcal{B}_{A}^{\mathrm{h}}+r^{5}\left(h_{r}^{\mathrm{q}} \mathcal{H}_{A}^{\mathrm{q}}+h_{r}^{\mathrm{h}} \mathcal{H}_{A}^{\mathrm{h}}\right)+O\left(r^{6}\right) \text {, } \\
& g_{A B}=r^{2} \Omega_{A B}\left[1+r^{2} e^{\mathrm{q}} \mathcal{E}^{\mathrm{a}}+r^{3} \dot{e}^{\mathrm{q}} \dot{\mathcal{E}}^{\mathrm{a}}+r^{4} \ddot{e}^{\mathrm{a}} \ddot{\mathcal{E}}^{\mathrm{a}}+r^{3} e^{\circ} \mathcal{E}^{\circ}+r^{4} \dot{e}^{\circ} \dot{\mathcal{E}}^{\circ}+r^{4} e^{\mathrm{h}} \mathcal{E}^{\mathrm{h}}+r^{4}\left(p^{\mathrm{m}} \mathcal{P}^{\mathrm{m}}+q^{\mathrm{m}} \mathcal{Q}^{\mathrm{m}}\right)\right. \\
& \left.+r^{4}\left(p^{\mathrm{q}} \mathcal{P}^{\mathrm{q}}+q^{\mathrm{q}} \mathcal{Q}^{\mathrm{q}}\right)+r^{4}\left(p^{\mathrm{h}} \mathcal{P}^{\mathrm{h}}+q^{\mathrm{h}} \mathcal{Q}^{\mathrm{h}}\right)+r^{4}\left(g^{\mathrm{d}} \mathcal{G}^{\mathrm{d}}+g^{\circ} \mathcal{G}^{\circ}\right)+O\left(r^{5}\right)\right]
\end{aligned}
$$

where, for example, $\mathcal{E}^{q}$ is the tidal potential introduced in Table III] and $e_{v v}^{q}, e_{v r}^{q}, e_{r r}^{q}$, and $e^{q}$ are the radial functions that come with it. An overdot on a tidal potential indicates differentiation with respect to $v$ - the time dependence is contained in the tidal moments - and the radial functions attached to time-differentiated potentials are also adorned with overdots (though these functions are not differentiated with respect to $v$ ).

The radial functions are determined by integrating the Einstein field equations in vacuum. The solutions are presented in Tables IX, X, XI, and XII. Integration for $\ell=0$ and $\ell=1$ requires some gauge choices. For $\ell=0$ we let $p_{v v}^{\mathrm{m}}$ and $p^{\mathrm{m}}$ be the two independent functions, and we set $p_{v r}^{\mathrm{m}}=-p_{v v}^{\mathrm{m}} / f$ and $p_{r r}^{\mathrm{m}}=2 p_{v v}^{\mathrm{m}} / f^{2}$, a choice inspired from the structure of the solutions for $\ell \geq 2$. For $\ell=1$ we set $g^{\mathrm{d}}=0$, and find that $g_{v v}^{\mathrm{d}}=0$ as a consequence of the field equations.

The radial functions depend on a number of integration constants, which are denoted with a sans-serif symbol such as $\mathrm{e}_{1}^{q}$. (Other constants of integration are fixed by demanding that the metric be well behaved at $r=2 M$. An exception to this rule concerns the terms proportional to $\mathrm{p}^{\mathrm{m}}$ and $\mathrm{q}^{\mathrm{m}}$; this point is discussed below.) These constants correspond to the freedom to redefine the tidal moments according to

$$
\mathcal{E}_{a b} \rightarrow \mathcal{E}_{a b}+\mathrm{e}_{1}^{\mathrm{q}} M \dot{\mathcal{E}}_{a b}+\mathrm{e}_{2}^{\mathrm{q}} M^{2} \ddot{\mathcal{E}}_{a b}+\mathrm{p}^{\mathrm{q}} M^{2} \mathcal{E}_{p\langle a} \mathcal{E}_{b\rangle}^{p}+\mathrm{q}^{\mathrm{p}} M^{2} \mathcal{B}_{p\langle a} \mathcal{B}^{p}{ }_{b\rangle},
$$




$$
\begin{aligned}
\mathcal{B}_{a b} & \rightarrow \mathcal{B}_{a b}+\mathrm{b}_{1}^{\mathrm{a}} M \dot{\mathcal{B}}_{a b}+\mathrm{b}_{2}^{\mathrm{a}} M^{2} \ddot{\mathcal{B}}_{a b}+\mathrm{h}^{\mathrm{a}} M^{2} \mathcal{E}_{p\langle a} \mathcal{B}^{p}{ }_{b\rangle}, \\
\mathcal{E}_{a b c} & \rightarrow \mathcal{E}_{a b c}+\mathrm{e}_{1}^{\circ} M \dot{\mathcal{E}}_{a b c}+\mathrm{g}^{\circ} M \epsilon_{p q\langle a} \mathcal{E}_{b}^{p} \mathcal{B}^{q}{ }_{c\rangle}, \\
\mathcal{B}_{a b c} & \rightarrow \mathcal{B}_{a b c}+\mathrm{b}_{1}^{\circ} M \dot{\mathcal{B}}_{a b c}
\end{aligned}
$$

and

$$
\begin{aligned}
2 \mathcal{E}_{a b c d} & \rightarrow 2 \mathcal{E}_{a b c d}+\mathrm{p}^{\mathrm{h}} \mathcal{E}_{\langle a b} \mathcal{E}_{c d\rangle}+\mathrm{q}^{\mathrm{h}} \mathcal{B}_{\langle a b} \mathcal{B}_{c d\rangle}, \\
\frac{10}{3} \mathcal{B}_{a b c d} & \rightarrow \frac{10}{3} \mathcal{B}_{a b c d}+\mathrm{h}^{\mathrm{h}} \mathcal{E}_{\langle a b} \mathcal{B}_{c d\rangle},
\end{aligned}
$$

as well as the freedom to redefine the mass parameter according to

$$
M \rightarrow M+\mathrm{p}^{\mathrm{m}} M^{5} \mathcal{E}_{p q} \mathcal{E}^{p q}+\mathrm{q}^{\mathrm{m}} M^{5} \mathcal{B}_{p q} \mathcal{B}^{p q} .
$$

More precisely stated, the radial functions presented in the Tables can be obtained from bare radial functions - the same functions with all integration constants set to zero - by applying the redefinitions of Eqs. (4.3), 44.4), and (4.5).

The freedom described by Eqs. 4.3 can be used to calibrate the tidal moments in a number of ways. For example, in Ref. 30] the freedom to redefine the tidal moments was exploited to ensure that in the light-cone gauge employed there, the event horizon of the deformed black hole continues to be situated at $r=2 M$. It was also exploited to ensure that the intrinsic geometry of the deformed horizon is independent of all $v$-derivatives of the tidal moments. In this "horizon calibration", the integration constants are fixed to

$$
\begin{aligned}
& \mathrm{e}_{1}^{\mathrm{q}}=-\frac{92}{15}, \quad \mathrm{e}_{2}^{\mathrm{q}}=\frac{5569}{225}, \quad \mathrm{e}_{1}^{\circ}=-\frac{188}{21}, \quad \mathrm{~b}_{1}^{\mathrm{q}}=-\frac{76}{15}, \quad \mathrm{~b}_{2}^{\mathrm{q}}=\frac{18553}{1050}, \quad \mathrm{~b}_{1}^{\circ}=-\frac{919}{105}, \\
& \mathrm{p}^{\mathrm{q}}=-\frac{2}{7}, \quad \mathrm{q}^{\mathrm{q}}=\frac{18}{7}, \quad \mathrm{~g}^{\circ}=-10, \quad \mathrm{~h}^{\mathrm{q}}=-\frac{44}{7} .
\end{aligned}
$$

The redefinitions of Eqs. (4.4 do not involve the black-hole mass $M$, and the freedom contained in these equations must be exploited to ensure that the tidal moments $\mathcal{E}_{a b c d}$ and $\mathcal{B}_{a b c d}$ that appear in the metric are those that are actually defined by Eqs. 2.3. This is achieved with

$$
\mathrm{p}^{\mathrm{h}}=\frac{355}{3}, \quad \mathrm{q}^{\mathrm{h}}=-\frac{5}{3}, \quad \mathrm{~h}^{\mathrm{h}}=-10 .
$$

Finally, the freedom described by Eq. 4.5) was also used by Poisson and Vlasov [30, to simplify the description of the event horizon. In this "horizon calibration" of the mass parameter,

$$
\mathrm{p}^{\mathrm{m}}=0, \quad \mathrm{q}^{\mathrm{m}}=0 .
$$

The results of Eqs. (4.6), (4.7), and (4.8) can all be established by showing that the perturbation presented here in Regge-Wheeler gauge can be obtained from the light-cone gauge of Poisson and Vlasov by a gauge transformation; this requires these specific values for the integration constants.

The "horizon calibration" is convenient for the purposes of examining the intrinsic geometry of the deformed horizon, but it may not be convenient for other purposes. An alternative calibration, designed to simplify the form of the radial functions, would be obtained by setting all integration constants (except for $\mathrm{p}^{\mathrm{h}}, \mathrm{q}^{\mathrm{h}}$, and $\mathrm{h}^{\mathrm{h}}$ ) to zero. Yet another choice is the "post-Newtonian calibration" to be introduced in Sec. V.

Some of the radial functions listed in the Tables have factors of $f:=1-2 M / r$ appearing in denominators, and this might suggest that these functions are not regular at $r=2 M$. Closer scrutiny, however, reveals that the functions are in fact regular. It can indeed be shown that $\ddot{e}_{v r}^{q}=O(f), \ddot{e}_{r r}^{q}=O(1)$, and $\ddot{b}_{r}^{q}=O(1)$ when $r \rightarrow 2 M$. The functions $p_{v r}^{\mathrm{m}}, p_{r r}^{\mathrm{m}}, q_{v r}^{\mathrm{m}}$, and $q_{r r}^{\mathrm{m}}$, however, are genuinely singular at $r=2 M$. The singular terms in the metric come with the integration constants $\mathrm{p}^{\mathrm{m}}$ and $\mathrm{q}^{\mathrm{m}}$, which represent the shift in mass parameter described by Eq. (4.5). These contributions to the monopole perturbation can easily be recast in a nonsingular form with a gauge transformation. But the (singular) gauge adopted here emerges as a natural choice when the metric is expressed in the $\left(t, r, \theta^{A}\right)$ coordinates of Sec. V] In any event, the choices of Eq. 4.8) ensure that the singular terms are eliminated from the metric.

\section{METRIC IN $(t, r, \theta, \phi)$ COORDINATES}

The metric in $\left(t, r, \theta^{A}\right)$ coordinates can be obtained from the metric of Eq. 4.2 by performing the coordinate transformation

$$
v=t+r \Delta, \quad \Delta:=1+\frac{2 M}{r} \ln \left(\frac{r}{2 M}-1\right),
$$


which implies that $d v=d t+f^{-1} d r$. In Sec. IV a tidal potential such as $\mathcal{E}^{q}$ was considered to be a function of $v$, and it must now be re-expressed in terms of $t$. Given our assumption that the tidal moments vary slowly, this can be done with the help of a Taylor expansion,

$$
\mathcal{E}^{\natural}(v)=\mathcal{E}^{\natural}(t)+r \Delta \dot{\mathcal{E}}^{\natural}(t)+\frac{1}{2} r^{2} \Delta^{2} \ddot{\mathcal{E}}^{\natural}(t)+O\left(r^{3}\right) .
$$

After making such substitutions and performing the coordinate transformation, we find that the metric of a tidally deformed, nonrotating black hole can also be expressed as

$$
\begin{aligned}
& g_{t t}=-f+r^{2} e_{t t}^{q} \mathcal{E}^{q}+r^{3} \dot{e}_{t t}^{\mathrm{q}} \dot{\mathcal{E}}^{\mathrm{q}}+r^{4} \ddot{e}_{t t}^{\mathrm{q}} \ddot{\mathcal{E}}^{\mathrm{q}}+r^{3} e_{t t}^{\circ} \mathcal{E}^{\circ}+r^{4} \dot{e}_{t t}^{\circ} \dot{\mathcal{E}}^{\circ}+r^{4} e_{t t}^{\mathrm{h}} \mathcal{E}^{\mathrm{h}}+r^{4}\left(p_{t t}^{\mathrm{m}} \mathcal{P}^{\mathrm{m}}+q_{t t}^{\mathrm{m}} \mathcal{Q}^{\mathrm{m}}\right) \\
& +r^{4}\left(p_{t t}^{\mathrm{a}} \mathcal{P}^{\mathrm{a}}+q_{t t}^{\mathrm{q}} \mathcal{Q}^{\mathrm{q}}\right)+r^{4}\left(p_{t t}^{\mathrm{h}} \mathcal{P}^{\mathrm{h}}+q_{t t}^{\mathrm{h}} \mathcal{Q}^{\mathrm{h}}\right)+r^{4}\left(g_{t t}^{\mathrm{d}} \mathcal{G}^{\mathrm{d}}+g_{t t}^{\circ} \mathcal{G}^{\circ}\right)+O\left(r^{5}\right), \\
& g_{t r}=r^{3} \dot{e}_{t r}^{\mathrm{q}} \dot{\mathcal{E}}^{\mathrm{a}}+r^{4} \ddot{e}_{t r}^{\mathrm{a}} \ddot{\mathcal{E}}^{\mathrm{a}}+r^{4} \dot{e}_{t r}^{\circ} \dot{\mathcal{E}}^{\circ}+r^{4}\left(g_{t r}^{\mathrm{d}} \mathcal{G}^{\mathrm{d}}+g_{t r}^{\circ} \mathcal{G}^{\circ}\right)+O\left(r^{5}\right) \text {, } \\
& g_{r r}=f^{-1}+r^{2} e_{r r}^{\mathrm{q}} \mathcal{E}^{\mathrm{q}}+r^{3} \dot{e}_{r r}^{\mathrm{q}} \dot{\mathcal{E}}^{\mathrm{q}}+r^{4} \ddot{e}_{r r}^{\mathrm{q}} \ddot{\mathcal{E}}^{\mathrm{q}}+r^{3} e_{r r}^{\circ} \mathcal{E}^{\circ}+r^{4} \dot{e}_{r r}^{\circ} \dot{\mathcal{E}}^{\circ}+r^{4} e_{r r}^{\mathrm{h}} \mathcal{E}^{\mathrm{h}}+r^{4}\left(p_{r r}^{\mathrm{m}} \mathcal{P}^{\mathrm{m}}+q_{r r}^{\mathrm{m}} \mathcal{Q}^{\mathrm{m}}\right) \\
& +r^{4}\left(p_{r r}^{\mathrm{q}} \mathcal{P}^{\mathrm{a}}+q_{r r}^{\mathrm{a}} \mathcal{Q}^{\mathrm{q}}\right)+r^{4}\left(p_{r r}^{\mathrm{h}} \mathcal{P}^{\mathrm{h}}+q_{r r}^{\mathrm{h}} \mathcal{Q}^{\mathrm{h}}\right)+r^{4}\left(g_{r r}^{\mathrm{d}} \mathcal{G}^{\mathrm{d}}+g_{r r}^{\circ} \mathcal{G}^{\circ}\right)+O\left(r^{5}\right), \\
& g_{t A}=r^{3} b_{t}^{\mathrm{q}} \mathcal{B}_{A}^{\mathrm{q}}+r^{4} \dot{b}_{t}^{\mathrm{q}} \dot{\mathcal{B}}_{A}^{\mathrm{q}}+r^{5} \ddot{b}_{t}^{\mathrm{q}} \ddot{\mathcal{B}}_{A}^{\mathrm{q}}+r^{4} b_{t}^{\circ} \mathcal{B}_{A}^{\circ}+r^{5} \dot{b}_{t}^{\circ} \dot{\mathcal{B}}_{A}^{\circ}+r^{5} b_{t}^{\mathrm{h}} \mathcal{B}_{A}^{\mathrm{h}}+r^{5}\left(h_{t}^{\mathrm{q}} \mathcal{H}_{A}^{\mathrm{q}}+h_{t}^{\mathrm{h}} \mathcal{H}_{A}^{\mathrm{h}}\right)+O\left(r^{6}\right) \text {, } \\
& g_{r A}=r^{4} \dot{b}_{r}^{\mathrm{q}} \dot{\mathcal{B}}_{A}^{\mathrm{q}}+r^{5} \ddot{b}_{r}^{\mathrm{q}} \ddot{\mathcal{B}}_{A}^{\mathrm{q}}+r^{5} \dot{b}_{r}^{\circ} \dot{\mathcal{B}}_{A}^{\circ}+r^{5}\left(h_{r}^{\mathrm{q}} \mathcal{H}_{A}^{\mathrm{q}}+h_{r}^{\mathrm{h}} \mathcal{H}_{A}^{\mathrm{h}}\right)+O\left(r^{6}\right) \text {, } \\
& g_{A B}=r^{2} \Omega_{A B}\left[1+r^{2} e^{\mathrm{q}} \mathcal{E}^{\mathrm{q}}+r^{3} \dot{e}^{\mathrm{q}} \dot{\mathcal{E}}^{\mathrm{a}}+r^{4} \ddot{e}^{\mathrm{q}} \ddot{\mathcal{E}}^{\mathrm{q}}+r^{3} e^{\circ} \mathcal{E}^{\circ}+r^{4} \dot{e}^{\circ} \dot{\mathcal{E}}^{\circ}+r^{4} e^{\mathrm{h}} \mathcal{E}^{\mathrm{h}}+r^{4}\left(p^{\mathrm{m}} \mathcal{P}^{\mathrm{m}}+q^{\mathrm{m}} \mathcal{Q}^{\mathrm{m}}\right)\right. \\
& \left.+r^{4}\left(p^{\mathrm{a}} \mathcal{P}^{\mathrm{a}}+q^{\mathrm{a}} \mathcal{Q}^{\mathrm{q}}\right)+r^{4}\left(p^{\mathrm{h}} \mathcal{P}^{\mathrm{h}}+q^{\mathrm{h}} \mathcal{Q}^{\mathrm{h}}\right)+r^{4}\left(g^{\mathrm{d}} \mathcal{G}^{\mathrm{d}}+g^{\circ} \mathcal{G}^{\circ}\right)+O\left(r^{5}\right)\right],
\end{aligned}
$$

where all tidal potentials are now given as functions of $t$. The new radial functions are listed in Tables XIII, XIV], XV] and XVI.

The freedom to redefine the tidal moments was introduced in Eqs. (4.3), (4.4), and (4.5), and the "horizon calibration" of these moments was introduced in Eqs. (4.6) and 4.8). For our purposes in Sec. VII, it is convenient to adopt an alternative "post-Newtonian calibration" that ensures that all radial functions begin an expansion in powers of $M / r$ with the largest power possible. For example, with a generic value for $\mathrm{e}_{1}^{q}$, the function $\dot{e}_{t t}^{q}$ possesses an expansion that begins at order $M / r$. Setting $\mathrm{e}_{1}^{\mathrm{q}}=-52 / 15$ eliminates this leading term, as well as terms of order $(M / r)^{2}$ and $(M / r)^{3}$, and leaves an expansion that begins at order $(M / r)^{6}$. This choice of integration constant therefore defines the post-Newtonian calibration for this radial function.

Adapting the procedure to all other radial functions, we find that the post-Newtonian calibration is achieved with

$$
\mathrm{e}_{1}^{\mathrm{q}}=-\frac{52}{15}, \quad \mathrm{e}_{1}^{\circ}=-\frac{30}{7}, \quad \mathrm{~b}_{1}^{\mathrm{q}}=-\frac{29}{10}, \quad \mathrm{~b}_{1}^{\circ}=-\frac{293}{70}, \quad \mathrm{~g}^{\circ}=0 .
$$

The values of $\mathrm{p}^{\mathrm{h}}, \mathrm{q}^{\mathrm{h}}$, and $\mathrm{h}^{\mathrm{h}}$ continue to be fixed by Eq. 4.7),

$$
\mathrm{p}^{\mathrm{h}}=\frac{355}{3}, \quad \mathrm{q}^{\mathrm{h}}=-\frac{5}{3}, \quad \mathrm{~h}^{\mathrm{h}}=-10 .
$$

And because the remaining constants do not affect the leading terms in expansions of the radial functions in powers of $M / r$, we set them to zero for simplicity:

$$
e_{2}^{q}=b_{2}^{q}=p^{m}=p^{q}=q^{m}=q^{q}=h^{q}=0
$$

\section{RELATION BETWEEN TIDAL MOMENTS}

Tidal moments corresponding to a calibration $\boldsymbol{\mu}$, where $\boldsymbol{\mu}$ collectively denotes a specific set of integration constants $\left\{\mathrm{e}_{1}^{\mathrm{q}}, \mathrm{e}_{2}^{\mathrm{q}}, \cdots, \mathrm{h}^{\mathrm{q}}, \mathrm{h}^{\mathrm{h}}\right\}$, are obtained from bare tidal moments through the redefinitions of Eqs. 4.3. We have that

$$
\begin{aligned}
\mathcal{E}_{a b}(\boldsymbol{\mu}) & =\mathcal{E}_{a b}(\mathbf{0})+\mathrm{e}_{1}^{\mathrm{q}} M \dot{\mathcal{E}}_{a b}(\mathbf{0})+\mathrm{e}_{2}^{\mathrm{q}} M^{2} \ddot{\mathcal{E}}_{a b}(\mathbf{0})+\mathrm{p}^{\mathrm{q}} M^{2} \mathcal{E}_{p\langle a} \mathcal{E}_{b\rangle}^{p}(\mathbf{0})+\mathrm{q}^{\mathrm{p}} M^{2} \mathcal{B}_{p\langle a} \mathcal{B}^{p}{ }_{b\rangle}(\mathbf{0}), \\
\mathcal{B}_{a b}(\boldsymbol{\mu}) & =\mathcal{B}_{a b}(\mathbf{0})+\mathrm{b}_{1}^{\mathrm{q}} M \dot{\mathcal{B}}_{a b}(\mathbf{0})+\mathrm{b}_{2}^{\mathrm{q}} M^{2} \ddot{\mathcal{B}}_{a b}(\mathbf{0})+\mathrm{h}^{\mathrm{q}} M^{2} \mathcal{E}_{p\langle a} \mathcal{B}_{b\rangle}^{p}(\mathbf{0}), \\
\mathcal{E}_{a b c}(\boldsymbol{\mu}) & =\mathcal{E}_{a b c}(\mathbf{0})+\mathrm{e}_{1}^{\circ} M \dot{\mathcal{E}}_{a b c}(\mathbf{0})+\mathrm{g}^{\circ} M \epsilon_{p q\langle a} \mathcal{E}_{b}^{p} \mathcal{B}^{q}{ }_{c\rangle}(\mathbf{0}), \\
\mathcal{B}_{a b c}(\boldsymbol{\mu}) & =\mathcal{B}_{a b c}(\mathbf{0})+\mathrm{b}_{1}^{\circ} M \dot{\mathcal{B}}_{a b c}(\mathbf{0}),
\end{aligned}
$$


where $\mathcal{E}_{a b}(\boldsymbol{\mu})$ and so on stand for the calibrated moments, while $\mathcal{E}_{a b}(\mathbf{0})$ and so on stand for the bare moments. It follows from these relations that tidal moments corresponding to different calibrations can be related to each other, independently of the bare moments. We have that

$$
\begin{aligned}
\mathcal{E}_{a b}\left(\boldsymbol{\mu}_{2}\right)= & \mathcal{E}_{a b}\left(\boldsymbol{\mu}_{1}\right)+\left[\mathrm{e}_{1}^{\mathrm{q}}(2)-\mathrm{e}_{1}^{\mathrm{q}}(1)\right] M \dot{\mathcal{E}}_{a b}\left(\boldsymbol{\mu}_{1}\right)+\left\{\mathrm{e}_{2}^{\mathrm{q}}(2)-\mathrm{e}_{2}^{\mathrm{q}}(1)-\mathrm{e}_{1}^{\mathrm{q}}(1)\left[\mathrm{e}_{1}^{\mathrm{q}}(2)-\mathrm{e}_{1}^{\mathrm{q}}(1)\right]\right\} M^{2} \ddot{\mathcal{E}}_{a b}\left(\boldsymbol{\mu}_{1}\right) \\
& +\left[\mathrm{p}^{\mathrm{q}}(2)-\mathrm{p}^{\mathrm{q}}(1)\right] M^{2} \mathcal{E}_{p\langle a} \mathcal{E}_{b\rangle}^{p}\left(\boldsymbol{\mu}_{1}\right)+\left[\mathrm{q}^{\mathrm{q}}(2)-\mathrm{p}^{\mathrm{q}}(1)\right] M^{2} \mathcal{B}_{p\langle a} \mathcal{B}^{p}{ }_{b\rangle}\left(\boldsymbol{\mu}_{1}\right), \\
\mathcal{B}_{a b}\left(\boldsymbol{\mu}_{2}\right)= & \mathcal{B}_{a b}\left(\boldsymbol{\mu}_{1}\right)+\left[\mathrm{b}_{1}^{\mathrm{q}}(2)-\mathrm{b}_{1}^{\mathrm{q}}(1)\right] M \dot{\mathcal{B}}_{a b}\left(\boldsymbol{\mu}_{1}\right)+\left\{\mathrm{b}_{2}^{\mathrm{q}}(2)-\mathrm{b}_{2}^{\mathrm{q}}(1)-\mathrm{b}_{1}^{\mathrm{q}}(1)\left[\mathrm{b}_{1}^{\mathrm{q}}(2)-\mathrm{b}_{1}^{\mathrm{q}}(1)\right]\right\} M^{2} \ddot{\mathcal{B}}_{a b}\left(\boldsymbol{\mu}_{1}\right) \\
& +\left[\mathrm{h}^{\mathrm{q}}(2)-\mathrm{h}^{\mathrm{q}}(1)\right] M^{2} \mathcal{E}_{p\langle a} \mathcal{B}^{p}{ }_{b\rangle}\left(\boldsymbol{\mu}_{1}\right), \\
\mathcal{E}_{a b c}\left(\boldsymbol{\mu}_{2}\right)= & \mathcal{E}_{a b c}\left(\boldsymbol{\mu}_{1}\right)+\left[\mathrm{e}_{1}^{\mathrm{o}}(2)-\mathrm{e}_{1}^{\circ}(1)\right] M \dot{\mathcal{E}}_{a b c}\left(\boldsymbol{\mu}_{1}\right)+\left[\mathrm{g}^{\circ}(2)-\mathrm{g}^{\circ}(1)\right] M \epsilon_{p q\langle a} \mathcal{E}_{b}^{p} \mathcal{B}_{c\rangle}^{q}\left(\boldsymbol{\mu}_{1}\right), \\
\mathcal{B}_{a b c}\left(\boldsymbol{\mu}_{2}\right)= & \mathcal{B}_{a b c}\left(\boldsymbol{\mu}_{1}\right)+\left[\mathrm{b}_{1}^{\circ}(2)-\mathrm{b}_{1}^{\circ}(1)\right] M \dot{\mathcal{B}}_{a b c}\left(\boldsymbol{\mu}_{1}\right),
\end{aligned}
$$

where constants such as $e_{1}^{q}(1)$ are elements of $\boldsymbol{\mu}_{1}$, while constants such as $e_{1}^{q}(2)$ are elements of $\boldsymbol{\mu}_{2}$. With these rules it follows from Eqs. (4.6), (4.7), (4.8), (5.4), (5.5), and (5.6) that the tidal moments in the horizon and post-Newtonian calibrations are related by

$$
\begin{aligned}
\mathcal{E}_{a b}(\mathrm{H}) & =\mathcal{E}_{a b}(\mathrm{PN})-\frac{8}{3} M \dot{\mathcal{E}}_{a b}(\mathrm{PN})+\frac{1163}{75} M^{2} \ddot{\mathcal{E}}_{a b}(\mathrm{PN})-\frac{2}{7} M^{2} \mathcal{E}_{p\langle a} \mathcal{E}_{b\rangle}^{p}(\mathrm{PN})+\frac{18}{7} M^{2} \mathcal{B}_{p\langle a} \mathcal{B}^{p}{ }_{b\rangle}(\mathrm{PN}) \\
\mathcal{B}_{a b}(\mathrm{H}) & =\mathcal{B}_{a b}(\mathrm{PN})-\frac{13}{6} M \dot{\mathcal{B}}_{a b}(\mathrm{PN})+\frac{23911}{2100} M^{2} \ddot{\mathcal{B}}_{a b}(\mathrm{PN})-\frac{44}{7} M^{2} \mathcal{E}_{p\langle a} \mathcal{B}_{b\rangle}^{p}(\mathrm{PN}) \\
\mathcal{E}_{a b c}(\mathrm{H}) & =\mathcal{E}_{a b c}(\mathrm{PN})-\frac{14}{3} M \dot{\mathcal{E}}_{a b c}(\mathrm{PN})-10 M \epsilon_{p q\langle a} \mathcal{E}_{b}^{p} \mathcal{B}^{q}{ }_{c\rangle}(\mathrm{PN}) \\
\mathcal{B}_{a b c}(\mathrm{H}) & =\mathcal{B}_{a b c}(\mathrm{PN})-\frac{137}{30} M \dot{\mathcal{B}}_{a b c}(\mathrm{PN}) .
\end{aligned}
$$

We recall that $\mathcal{E}_{a b c d}$ and $\mathcal{B}_{a b c d}$ have a fixed calibration determined by the definitions of Eqs. 2.3.

\section{POST-NEWTONIAN POTENTIALS OF A TIDALLY DEFORMED BLACK HOLE}

In this section we obtain the post-Newtonian limit of the metric of a tidally deformed black hole. We shall show that with a shift of the radial coordinate and a transformation to Cartesian coordinates, it can be expressed in the standard form (see, for example, Sec. 8.2 of Ref. [34])

$$
\begin{aligned}
& g_{t t}=-1+2 U+2\left(\Psi-U^{2}\right)+2 \mathrm{PN}, \\
& g_{t a}=-4 U_{a}+2 \mathrm{PN}, \\
& g_{a b}=(1+2 U) \delta_{a b}+2 \mathrm{PN},
\end{aligned}
$$

in terms of a Newtonian potential $U$, a vector potential $U_{a}$, and a post-Newtonian potential $\Psi$. This exercise prepares the way for the matching calculation of Sec. VIII

The integration constants of Eqs. (5.4), (5.5), and (5.6) — which define the "post-Newtonian calibration" of the tidal moments - are inserted in the metric of Eqs. (5.3), which is then expressed as a post-Newtonian expansion truncated after the first post-Newtonian $(1 \mathrm{PN})$ order. The gravitoelectric tidal moments are themselves given post-Newtonian expansions of the form

$$
\begin{aligned}
\mathcal{E}_{a b} & =\mathcal{E}_{a b}(0 \mathrm{PN})+\mathcal{E}_{a b}(1 \mathrm{PN})+2 \mathrm{PN} \\
\mathcal{E}_{a b c} & =\mathcal{E}_{a b c}(0 \mathrm{PN})+\mathcal{E}_{a b c}(1 \mathrm{PN})+2 \mathrm{PN} \\
\mathcal{E}_{a b c d} & =\mathcal{E}_{a b c d}(0 \mathrm{PN})+\mathcal{E}_{a b c d}(1 \mathrm{PN})+2 \mathrm{PN},
\end{aligned}
$$

while the gravitomagnetic moments are truncated at their leading, 1PN order. The metric is expressed in terms of the harmonic radial coordinate

$$
\bar{r}=r-M,
$$

where $r$ is the usual areal coordinate employed previously; this shift by $-M$ is a post-Newtonian correction.

With all this the metric becomes

$$
g_{t t}=-1+\left[\frac{2 M}{\bar{r}}-\bar{r}^{2} \mathcal{E}^{\mathrm{q}}(0 \mathrm{PN})-\frac{1}{3} \bar{r}^{3} \mathcal{E}^{\circ}(0 \mathrm{PN})-\frac{1}{12} \bar{r}^{4} \mathcal{E}^{\mathrm{h}}(0 \mathrm{PN})\right]
$$




$$
\begin{aligned}
& +\left[-\frac{2 M^{2}}{\bar{r}^{2}}+2 M \bar{r} \mathcal{E}^{\mathrm{q}}(0 \mathrm{PN})+\frac{2}{3} M \bar{r}^{2} \mathcal{E}^{\circ}(0 \mathrm{PN})+\frac{1}{6} M \bar{r}^{3} \mathcal{E}^{\mathrm{h}}(0 \mathrm{PN})-\frac{11}{42} \bar{r}^{4} \ddot{\mathcal{E}}^{\mathrm{q}}(0 \mathrm{PN})\right. \\
& \left.-\bar{r}^{2} \mathcal{E}^{\mathrm{q}}(1 \mathrm{PN})-\frac{1}{3} \bar{r}^{3} \mathcal{E}^{\circ}(1 \mathrm{PN})-\frac{1}{12} \bar{r}^{4} \mathcal{E}^{\mathrm{h}}(1 \mathrm{PN})-\frac{1}{15} \bar{r}^{4} \mathcal{P}^{\mathrm{m}}-\frac{2}{7} \bar{r}^{4} \mathcal{P}^{\mathrm{q}}+\frac{1}{3} \bar{r}^{4} \mathcal{P}^{\mathrm{h}}\right]+O\left(\bar{r}^{5}, 2 \mathrm{PN}\right), \\
g_{t r}= & -\frac{2}{3} \bar{r}^{3} \dot{\mathcal{E}}^{\mathrm{q}}(0 \mathrm{PN})-\frac{1}{6} \bar{r}^{4} \dot{\mathcal{E}}^{\circ}(0 \mathrm{PN})+O\left(\bar{r}^{5}, 2 \mathrm{PN}\right), \\
g_{r r}= & 1+\left[\frac{2 M}{\bar{r}}-\bar{r}^{2} \mathcal{E}^{\mathrm{q}}(0 \mathrm{PN})-\frac{1}{3} \bar{r}^{3} \mathcal{E}^{\circ}(0 \mathrm{PN})-\frac{1}{12} \bar{r}^{4} \mathcal{E}^{\mathrm{h}}(0 \mathrm{PN})\right]+O\left(\bar{r}^{5}, 2 \mathrm{PN}\right) \\
g_{t A}= & \frac{2}{3} \bar{r}^{3} \mathcal{B}_{A}^{\mathrm{q}}+\frac{1}{4} \bar{r}^{4} \mathcal{B}_{A}^{\circ}+\frac{1}{15} \bar{r}^{5} \mathcal{B}_{A}^{\mathrm{h}}+O\left(\bar{r}^{6}, 2 \mathrm{PN}\right), \\
g_{r A}= & 2 \mathrm{PN}, \\
g_{A B}= & r^{2} \Omega_{A B}+r^{2} \Omega_{A B}\left[\frac{2 M}{\bar{r}}-\bar{r}^{2} \mathcal{E}^{\mathrm{q}}(0 \mathrm{PN})-\frac{1}{3} \bar{r}^{3} \mathcal{E}^{\circ}(0 \mathrm{PN})-\frac{1}{12} \bar{r}^{4} \mathcal{E}^{\mathrm{h}}(0 \mathrm{PN})\right]+O\left(\bar{r}^{7}, 2 \mathrm{PN}\right) .
\end{aligned}
$$

In $g_{t t}$, the first set of terms within square brackets are of Newtonian $(0 \mathrm{PN})$ order, while the second set of terms are 1PN corrections. It is understood that in the post-Newtonian terms, the tidal potentials $\mathcal{P}^{\mathrm{m}}, \mathcal{P}^{\mathrm{q}}$, and $\mathcal{P}^{\mathrm{h}}$ are constructed from $\mathcal{E}_{a b}(0 \mathrm{PN})$.

Conversion to Cartesian coordinates $x^{a}=\bar{r} \Omega^{a}\left(\theta^{A}\right)$ gives rise to the standard post-Newtonian form of the metric, as given by Eqs. (7.1), with

$$
\begin{aligned}
U= & \frac{M}{\bar{r}}-\frac{1}{2} \bar{r}^{2} \mathcal{E}^{\mathrm{q}}(0 \mathrm{PN})-\frac{1}{6} \bar{r}^{3} \mathcal{E}^{\circ}(0 \mathrm{PN})-\frac{1}{24} \bar{r}^{4} \mathcal{E}^{\mathrm{h}}(0 \mathrm{PN})+O\left(\bar{r}^{5}\right), \\
\Psi-U^{2}= & -\frac{M^{2}}{\bar{r}^{2}}+M \bar{r} \mathcal{E}^{\mathrm{q}}(0 \mathrm{PN})+\frac{1}{3} M \bar{r}^{2} \mathcal{E}^{\circ}(0 \mathrm{PN})+\frac{1}{12} M \bar{r}^{3} \mathcal{E}^{\mathrm{h}}(0 \mathrm{PN})-\frac{11}{84} \bar{r}^{4} \ddot{\mathcal{E}}^{\mathrm{q}}(0 \mathrm{PN}) \\
& -\frac{1}{2} \bar{r}^{2} \mathcal{E}^{\mathrm{q}}(1 \mathrm{PN})-\frac{1}{6} \bar{r}^{3} \mathcal{E}^{\circ}(1 \mathrm{PN})-\frac{1}{24} \bar{r}^{4} \mathcal{E}^{\mathrm{h}}(1 \mathrm{PN})-\frac{1}{30} \bar{r}^{4} \mathcal{P}^{\mathrm{m}}-\frac{1}{7} \bar{r}^{4} \mathcal{P}^{\mathrm{q}}+\frac{1}{6} \bar{r}^{4} \mathcal{P}^{\mathrm{h}}+O\left(\bar{r}^{5}\right), \\
U_{a}= & -\frac{1}{6} \bar{r}^{2} \mathcal{B}_{a}^{\mathrm{q}}-\frac{1}{16} \bar{r}^{3} \mathcal{B}_{a}^{\circ}-\frac{1}{60} \bar{r}^{4} \mathcal{B}_{a}^{\mathrm{h}}+\frac{1}{6} \bar{r}^{3} \Omega_{a} \dot{\mathcal{E}}^{\mathrm{q}}(0 \mathrm{PN})+\frac{1}{24} \bar{r}^{4} \Omega_{a} \dot{\mathcal{E}}^{\circ}(0 \mathrm{PN})+O\left(\bar{r}^{5}\right) .
\end{aligned}
$$

That the standard post-Newtonian form of the metric is recovered in our calculation is the reason for employing $\bar{r}$ as a radial coordinate; this form would not be achieved in the original radial coordinate $r$. It should be noted that in spite of our use of $\bar{r}$, the coordinates $x^{a}$ are not harmonic: the potentials do not satisfy the harmonic condition $\partial_{t} U+\partial_{a} U^{a}=0$. The reason for this, of course, is that the metric perturbation is presented in Regge-Wheeler gauge instead of a harmonic gauge.

The square of the Newtonian potential $U$ can be calculated with the help of the identity $\mathcal{E}^{q} \mathcal{E}^{q}=\frac{2}{15} \mathcal{P}^{\mathrm{m}}+\frac{4}{7} \mathcal{P}^{\mathrm{q}}+\mathcal{P}^{\mathrm{h}}$, and this reveals that the post-Newtonian potential is given by

$$
\Psi=-\frac{1}{2} \bar{r}^{2} \mathcal{E}^{\mathrm{q}}(1 \mathrm{PN})-\frac{1}{6} \bar{r}^{3} \mathcal{E}^{\circ}(1 \mathrm{PN})-\frac{1}{24} \bar{r}^{4} \mathcal{E}^{\mathrm{h}}(1 \mathrm{PN})-\frac{11}{84} \bar{r}^{4} \ddot{\mathcal{E}}^{\mathrm{q}}(0 \mathrm{PN})+\frac{5}{12} \bar{r}^{4} \mathcal{P}^{\mathrm{h}}+O\left(\bar{r}^{5}\right) .
$$

Notice that $\Psi$ does not involve $\mathcal{P}^{\mathrm{m}}$ and $\mathcal{P}^{\mathrm{q}}$, which have cancelled out in these manipulations.

To obtain the final form of the potentials we insert the definitions of the tidal potentials in terms of the tidal moments. This yields

$$
\begin{aligned}
& \bar{U}=\frac{M}{\bar{r}}-\frac{1}{2} \overline{\mathcal{E}}_{a b}(0 \mathrm{PN}) \bar{x}^{a} \bar{x}^{b}-\frac{1}{6} \overline{\mathcal{E}}_{a b c}(0 \mathrm{PN}) \bar{x}^{a} \bar{x}^{b} \bar{x}^{c}-\frac{1}{12} \overline{\mathcal{E}}_{a b c d}(0 \mathrm{PN}) \bar{x}^{a} \bar{x}^{b} \bar{x}^{c} \bar{x}^{d}+O\left(\bar{r}^{5}\right), \\
& \bar{U}_{a}=-\frac{1}{6} \epsilon_{a p q} \bar{x}^{p} \overline{\mathcal{B}}^{q} \bar{x}^{b}-\frac{1}{12} \epsilon_{a p q} \bar{x}^{p} \overline{\mathcal{B}}_{b c}^{q} \bar{x}^{b} \bar{x}^{c}-\frac{1}{18} \epsilon_{a p q} \bar{x}^{p} \overline{\mathcal{B}}_{b c d}^{q} \bar{x}^{b} \bar{x}^{c} \bar{x}^{d} \\
& +\frac{1}{6} \bar{x}_{a} \dot{\overline{\mathcal{E}}}_{b c}(0 \mathrm{PN}) \bar{x}^{b} \bar{x}^{c}+\frac{1}{24} \bar{x}_{a} \dot{\overline{\mathcal{E}}}_{b c d}(0 \mathrm{PN}) \bar{x}^{b} \bar{x}^{c} \bar{x}^{d}+O\left(\bar{r}^{5}\right), \\
& \bar{\Psi}=-\frac{1}{2} \overline{\mathcal{E}}_{a b}(1 \mathrm{PN}) \bar{x}^{a} \bar{x}^{b}-\frac{1}{6} \overline{\mathcal{E}}_{a b c}(1 \mathrm{PN}) \bar{x}^{a} \bar{x}^{b} \bar{x}^{c}-\frac{1}{12} \overline{\mathcal{E}}_{a b c d}(1 \mathrm{PN}) \bar{x}^{a} \bar{x}^{b} \bar{x}^{c} \bar{x}^{d} \\
& -\frac{11}{84} \bar{r}^{2} \ddot{\overline{\mathcal{E}}}_{a b}(0 \mathrm{PN}) \bar{x}^{a} \bar{x}^{b}+\frac{5}{12} \overline{\mathcal{E}}_{\langle a b} \overline{\mathcal{E}}_{c d\rangle}(0 \mathrm{PN}) \bar{x}^{a} \bar{x}^{b} \bar{x}^{c} \bar{x}^{d}+O\left(\bar{r}^{5}\right) .
\end{aligned}
$$

We have placed overbars on the potentials, tidal moments, and coordinates in anticipation of the developments of Sec. VIII in this notation the tidal moments are functions of time $\bar{t}$. The overbars indicate that the potentials, tidal moments, and coordinates refer to the black hole's reference frame, which is to be distinguished from the post-Newtonian barycentric frame to be introduced below. 
It is straightforward to show that the potentials satisfy the post-Newtonian field equations

$$
\begin{aligned}
& 0=\bar{\nabla}^{2} \bar{U}, \\
& 0=\bar{\nabla}^{2} \bar{\Psi}+3 \partial_{\bar{t} \bar{U}} \bar{U}+4 \partial_{\bar{t} \bar{a}} \bar{U}^{a}, \\
& 0=\bar{\nabla}^{2} \bar{U}_{a}-\partial_{\bar{t} \bar{a}} \bar{U}-\partial_{\bar{a} \bar{b}} \bar{U}^{b} .
\end{aligned}
$$

These reduce to the standard post-Newtonian equations (Sec. 8.2 of Ref. 34]) when the potentials satisfy the harmonic condition $\partial_{\bar{t}} \bar{U}+\partial_{\bar{a}} \bar{U}^{a}=0$.

\section{MATCHING TO A POST-NEWTONIAN METRIC}

The metric of a tidally deformed black hole was constructed in Secs. IV] and V] and it was expressed in terms of tidal moments $\overline{\mathcal{E}}_{a b}, \overline{\mathcal{E}}_{a b c}, \overline{\mathcal{E}}_{a b c d}, \overline{\mathcal{B}}_{a b}, \overline{\mathcal{B}}_{a b c}, \overline{\mathcal{B}}_{a b c d}$. (See the remark regarding the overbar at the end of Sec. VII.) The tidal moments occur in the metric as freely specifiable functions of time, and these cannot be determined by solving the Einstein field equations in a domain limited to the black hole's immediate neighborhood. Their determination must instead rely on matching the black-hole metric to another metric that incorporates all relevant information regarding the black hole's remote environment. We achieve this in this section, taking the black hole to be a member of a post-Newtonian system containing any number of external bodies. In this treatment, the black hole's internal gravity is allowed to be strong, but the mutual gravity between black hole and external bodies is assumed to be sufficiently weak to be adequately described by a post-Newtonian expansion of the metric. The matching between the black-hole and post-Newtonian metrics will determine the tidal moments, which are calculated accurately through the first post-Newtonian (1PN) approximation.

\section{A. Barycentric potentials}

The black hole is taken to be a member of a post-Newtonian system of gravitating bodies. The metric is given by Eq. (7.1), in terms of harmonic coordinates $\left(t, x^{a}\right)$ attached to the system's barycenter, and in terms of barycentric potentials $U, U_{j}$, and $\Psi$. The post-Newtonian metric is valid in a domain that contains all bodies but leaves out a sphere of radius $\bar{r} \gg M$ surrounding the black hole; this region is excluded because the black hole's internal gravity is too strong to be adequately captured by a post-Newtonian expansion. As illustrated in Fig. 11, there exists an overlap region in which the black-hole metric of Sec. V] and the post-Newtonian metric are both valid; the matching of the metrics takes place in this region.

It is helpful to define new post-Newtonian potentials $\psi$ and $X$ by the relation $\Psi=\psi+\frac{1}{2} \partial_{t t} X$. In the vacuum region between bodies, the potentials satisfy the post-Newtonian field equations

$$
\nabla^{2} U=0, \quad \nabla^{2} U_{j}=0, \quad \nabla^{2} \psi=0, \quad \nabla^{2} X=2 U,
$$

as well as the harmonic condition

$$
\partial_{t} U+\partial_{j} U^{j}=0
$$

Each equation is linear, and a solution describing a black hole and a collection of external bodies can be obtained by linear superposition. We model the black hole as a post-Newtonian monopole of mass $M$ at position $\boldsymbol{x}=\boldsymbol{r}(t)$ in the barycentric frame. We let $\boldsymbol{v}:=d \boldsymbol{r} / d t$ be the black hole's velocity vector, and $\boldsymbol{a}:=d \boldsymbol{v} / d t$ be its acceleration vector. The potentials are written as

$$
\begin{aligned}
U(t, \boldsymbol{x}) & =\frac{M}{s}+U_{\mathrm{ext}}(t, \boldsymbol{x}), \\
U^{j}(t, \boldsymbol{x}) & =\frac{M v^{j}}{s}+U_{\mathrm{ext}}^{j}(t, \boldsymbol{x}), \\
\psi(t, \boldsymbol{x}) & =\frac{M \mu}{s}+\psi_{\mathrm{ext}}(t, \boldsymbol{x}), \\
X(t, \boldsymbol{x}) & =M s+X_{\mathrm{ext}}(t, \boldsymbol{x}),
\end{aligned}
$$

where $s:=|\boldsymbol{x}-\boldsymbol{r}|$ is the Euclidean distance between the black hole and a field point at $\boldsymbol{x}$, while $U_{\text {ext }}, U_{\text {ext }}^{j}, \psi_{\text {ext }}$, and $X_{\text {ext }}$ are the potentials created by the external bodies; these separately satisfy the field equations of Eq. (8.1) and 
the harmonic condition of Eq. 8.2). The arbitrary function $\mu(t)$ represents a post-Newtonian correction to the mass parameter. It cannot be determined by integrating the post-Newtonian field equations in a domain that excludes the black hole, and must instead be obtained by matching the post-Newtonian metric with the black-hole metric of Sec. V]

Inserting $\psi$ and $X$ in the expression for $\Psi$ returns

$$
\Psi(t, \boldsymbol{x})=-\frac{M}{2 s^{3}}(\boldsymbol{v} \cdot \boldsymbol{s})^{2}+\frac{M}{s}\left(\mu+\frac{1}{2} v^{2}\right)-\frac{M}{2 s} \boldsymbol{a} \cdot \boldsymbol{s}+\Psi_{\mathrm{ext}}(t, \boldsymbol{x}),
$$

where $\boldsymbol{s}:=\boldsymbol{x}-\boldsymbol{r}, v^{2}:=\boldsymbol{v} \cdot \boldsymbol{v}$, and $\Psi_{\mathrm{ext}}:=\psi_{\mathrm{ext}}+\frac{1}{2} \partial_{t t} X_{\mathrm{ext}}$.

\section{B. Transformation to the black-hole frame}

The post-Newtonian metric of the preceding subsection was presented in coordinates $\left(t, x^{a}\right)$ attached to the barycentric frame of the entire post-Newtonian system. On the other hand, the black-hole metric of Sec. $\mathrm{V}]$ was presented in coordinates $\left(\bar{t}, \bar{x}^{a}\right)$ that are attached to the black hole's own reference frame, which is moving relative to the barycentric frame. To match the metrics we must transform the post-Newtonian potentials to the coordinates $\left(\bar{t}, \bar{x}^{a}\right)$, and compare the expressions with Eqs. (7.7).

The systems $\left(t, x^{a}\right)$ and $\left(\bar{t}, \bar{x}^{a}\right)$ are both compatible with the standard form of the post-Newtonian metric, and the coordinate transformation relating two such systems is presented in Sec. 8.3 of Ref. 34 (a summary of work previously carried out in Ref. [35]). It should be noted that while $\left(t, x^{a}\right)$ is a system of harmonic coordinates, the $\left(\bar{t}, \bar{x}^{a}\right)$ coordinates are not harmonic. The transformation described in Ref. 34 applies to such situations, but the reader should be warned that the summary of Box 8.2 refers strictly to two systems of harmonic coordinates.

The coordinate transformation is characterized by arbitrary functions $A(\bar{t}), H^{a}(\bar{t}), R^{a}(\bar{t})$, and $\beta\left(\bar{t}, \bar{x}^{a}\right)$ in addition to the black-hole position vector $r^{a}(\bar{t})$, here expressed as a function of the barred time coordinate. The transformation is given by

$$
t=\bar{t}+\left(A+v_{a} \bar{x}^{a}\right)+\beta+3 \mathrm{PN}, \quad x^{a}=\bar{x}^{a}+r^{a}+\left(H^{a}+H_{b}^{a} \bar{x}^{b}+\frac{1}{2} H_{b c}^{a} \bar{x}^{b} \bar{x}^{c}\right)+2 \mathrm{PN},
$$

where $H_{a b}:=\epsilon_{a b c} R^{c}+\frac{1}{2} v_{a} v_{b}-\left(\dot{A}-\frac{1}{2} v^{2}\right) \delta_{a b}$ and $H_{a b c}:=-\delta_{a b} a_{c}-\delta_{a c} a_{b}+\delta_{b c} a_{a}$, with $v^{a}:=d r^{a} / d \bar{t}$ and $a^{a}:=d v^{a} / d \bar{t}$; an overdot indicates differentiation with respect to $\bar{t}$. The bracketed terms in the equation for $t$ represent a $1 \mathrm{PN}$ adjustment to the time coordinate that impacts the Newtonian potential $U$, while $\beta$ represents a $2 \mathrm{PN}$ adjustment that impacts the vector and post-Newtonian potentials. The bracketed terms in the equation for $x^{a}$ are of $1 \mathrm{PN}$ order. In the following the acceleration vector is decomposed into Newtonian and post-Newtonian terms,

$$
a^{a}=a^{a}(0 \mathrm{PN})+a^{a}(1 \mathrm{PN})+2 \mathrm{PN} ;
$$

the acceleration that appears in $H_{a b c}$ can be truncated at Newtonian order.

The transformed potentials $\bar{U}, \bar{U}^{j}$, and $\bar{\Psi}$, those of the black-hole frame, are expressed in terms of "hatted potentials" $\hat{U}, \hat{U}^{j}$, and $\hat{\Psi}$, related to the original potentials $U, U^{j}$, and $\Psi$ by equations of the form

$$
\hat{U}(\bar{t}, \overline{\boldsymbol{x}}):=U(t=\bar{t}, \boldsymbol{x}=\overline{\boldsymbol{x}}+\boldsymbol{r}(\bar{t})) ;
$$

the hatted potentials are therefore equal to the original potentials evaluated at the time $\bar{t}$ and position $\overline{\boldsymbol{x}}+\boldsymbol{r}$. Because of the time dependence contained in $\boldsymbol{r}(\bar{t})$, the time derivative of a hatted potential is related by

$$
\partial_{\bar{t}} \hat{U}=\partial_{t} U+v^{a} \partial_{a} U
$$

to derivatives of the original potential; it is understood that the right-hand side of this equation is evaluated at $t=\bar{t}$ and $x^{a}=\bar{x}^{a}+r^{a}(\bar{t})$. Spatial derivatives are related by $\partial_{\bar{a}} \hat{U}=\partial_{a} U$, where again the right-hand side is evaluated at the new time and position.

By virtue of these differentiation rules, the harmonic condition satisfied by the hatted potentials takes the form

$$
\partial_{\bar{t}} \hat{U}-v^{a} \partial_{\bar{a}} \hat{U}+\partial_{\bar{a}} \hat{U}^{a}=0 .
$$

The field equations satisfied by the Newtonian and vector potentials become

$$
\bar{\nabla}^{2} \hat{U}=0, \quad \bar{\nabla}^{2} \hat{U}^{j}=0,
$$


while the field equation satisfied by $\Psi, \nabla^{2} \Psi-\partial_{t t} U=0$, takes the new form

$$
\bar{\nabla}^{2} \hat{\Psi}-\partial_{\bar{t} \bar{t}} \hat{U}+2 v^{a} \partial_{\bar{t} \bar{a}} \hat{U}+a^{a} \partial_{\bar{a}} \hat{U}-v^{a} v^{b} \partial_{\bar{a} \bar{b}} \hat{U}=0
$$

We recall that the harmonic condition and the field equations are satisfied separately by the external potentials $\hat{U}_{\text {ext }}$, $\hat{U}_{\text {ext }}^{j}$, and $\hat{\Psi}_{\text {ext }}$.

We take advantage of the large separation between the black hole and all external bodies to express each external potential as a Taylor expansion about $\bar{x}^{a}=0$. We write, for example,

$$
\begin{aligned}
\hat{U}_{\text {ext }}(\bar{t}, \overline{\boldsymbol{x}})= & \hat{U}_{\text {ext }}(\bar{t}, \mathbf{0})+\bar{x}^{a} \partial_{\bar{a}} \hat{U}_{\text {ext }}(\bar{t}, \mathbf{0})+\frac{1}{2} \bar{x}^{a} \bar{x}^{b} \partial_{\bar{a} \bar{b}} \hat{U}_{\text {ext }}(\bar{t}, \mathbf{0})+\frac{1}{6} \bar{x}^{a} \bar{x}^{b} \bar{x}^{c} \partial_{\bar{a} \bar{b} \bar{c}} \hat{U}_{\text {ext }}(\bar{t}, \mathbf{0}) \\
& +\frac{1}{24} \bar{x}^{a} \bar{x}^{b} \bar{x}^{c} \bar{x}^{d} \partial_{\bar{a} \bar{b} \bar{c} \bar{d}} \hat{U}_{\text {ext }}(\bar{t}, \mathbf{0})+O\left(\bar{r}^{5}\right)
\end{aligned}
$$

where $\bar{r}:=|\overline{\boldsymbol{x}}|$. In all equations that appear below, the external potentials and their derivatives shall be evaluated at $\bar{x}^{a}=0$. In a similar way we express the function $\beta$ that appears in the coordinate transformation as a Taylor expansion of the form

$$
\beta(\bar{t}, \overline{\boldsymbol{x}})={ }_{0} \beta(\bar{t})+{ }_{1} \beta_{a}(\bar{t}) \bar{x}^{a}+\frac{1}{2}{ }_{2} \beta_{a b}(\bar{t}) \bar{x}^{a} \bar{x}^{b}+\frac{1}{6}{ }_{3} \beta_{a b c}(\bar{t}) \bar{x}^{a} \bar{x}^{b} \bar{x}^{c}+\frac{1}{24}{ }_{4} \beta_{a b c d}(\bar{t}) \bar{x}^{a} \bar{x}^{b} \bar{x}^{c} \bar{x}^{d}+O\left(\bar{r}^{5}\right),
$$

in which the expansion coefficients are fully symmetric tensors; the number that appears before each tensor symbol indicates the associated power of $\bar{r}$ in the expansion.

With all these ingredients in place, a long but straightforward computation reveals that the equations listed in Secs. 8.3.2 and 8.3.3 of Ref. [34] become

$$
\begin{aligned}
\bar{U}= & \frac{M}{\bar{r}}+{ }_{0} \bar{U}+{ }_{1} \bar{U}_{a} \bar{x}^{a}+\frac{1}{2}{ }_{2} \bar{U}_{a b} \bar{x}^{a} \bar{x}^{b}+\frac{1}{6}{ }_{3} \bar{U}_{a b c} \bar{x}^{a} \bar{x}^{b} \bar{x}^{c}+\frac{1}{24}{ }_{4} \bar{U}_{a b c d} \bar{x}^{a} \bar{x}^{b} \bar{x}^{c} \bar{x}^{d}+O\left(\bar{r}^{5}\right), \\
\bar{U}_{j}= & { }_{0} \bar{U}_{j}+{ }_{1} \bar{U}_{j a} \bar{x}^{a}+\frac{1}{2}{ }_{2} \bar{U}_{j a b} \bar{x}^{a} \bar{x}^{b}+\frac{1}{6}{ }_{3} \bar{U}_{j a b c} \bar{x}^{a} \bar{x}^{b} \bar{x}^{c}+\frac{1}{24}{ }_{4} \bar{U}_{j a b c d} \bar{x}^{a} \bar{x}^{b} \bar{x}^{c} \bar{x}^{d}+O\left(\bar{r}^{5}\right), \\
\bar{\Psi}= & -\frac{M}{\bar{r}^{3}} F_{a} \bar{x}^{a}+\frac{M}{\bar{r}}\left(\dot{A}-2 v^{2}+\mu\right)+{ }_{0} \bar{\Psi}+{ }_{1} \bar{\Psi}_{a} \bar{x}^{a}+\frac{1}{2}{ }_{2} \bar{\Psi}_{a b} \bar{x}^{a} \bar{x}^{b}+\frac{1}{6}{ }_{3} \bar{\Psi}_{a b c} \bar{x}^{a} \bar{x}^{b} \bar{x}^{c} \\
& +\frac{1}{24}{ }_{4} \bar{\Psi}_{a b c d} \bar{x}^{a} \bar{x}^{b} \bar{x}^{c} \bar{x}^{d}+O\left(\bar{r}^{5}\right),
\end{aligned}
$$

where $F_{a}:=H_{a}-A v_{a}$ and the remaining expansion coefficients are given by

$$
\begin{aligned}
{ }_{0} \bar{U} & =\hat{U}_{\text {ext }}-\dot{A}+\frac{1}{2} v^{2}, \\
{ }_{1} \bar{U}_{a} & =\partial_{\bar{a}} \hat{U}_{\text {ext }}-a_{a}(0 \mathrm{PN}), \\
{ }_{2} \bar{U}_{a b} & =\partial_{\bar{a} \bar{b}} \hat{U}_{\mathrm{ext}}, \\
{ }_{3} \bar{U}_{a b c} & =\partial_{\bar{a} \bar{b} \bar{c}} \hat{U}_{\mathrm{ext}}, \\
{ }_{4} \bar{U}_{a b c d} & =\partial_{\bar{a} \bar{b} \bar{c} \bar{d}} \hat{U}_{\mathrm{ext}}
\end{aligned}
$$

$$
\begin{aligned}
& 4_{0} \bar{U}_{j}=4 \hat{P}_{j}+\left(2 \dot{A}-v^{2}\right) v_{j}-\dot{H}_{j}+\epsilon_{j p q} v^{p} R^{q}+{ }_{1} \beta_{j}, \\
& 4_{1} \bar{U}_{j a}=4 \partial_{\bar{a}} \hat{P}_{j}+\frac{3}{2} v_{j} a_{a}+\frac{1}{2} a_{j} v_{a}+\left(\ddot{A}-2 v_{p} a^{p}\right) \delta_{j a}-\epsilon_{j a p} \dot{R}^{p}+{ }_{2} \beta_{j a}, \\
& 4_{2} \bar{U}_{j a b}=4 \partial_{\bar{a} \bar{b}} \hat{P}_{j}+2 \delta_{j(a} \dot{a}_{b)}-\delta_{a b} \dot{a}_{j}+{ }_{3} \beta_{j a b}, \\
& { }_{4}{ }_{3} \bar{U}_{j a b c}=4 \partial_{\bar{a} \bar{b} \bar{c}} \hat{P}_{j}+{ }_{4} \beta_{j a b c}, \\
& { }_{4}{ }_{4} \bar{U}_{j a b c d}=4 \partial_{\bar{a} \bar{b} \bar{c} \bar{d}} \hat{P}_{j}+{ }_{5} \beta_{j a b c d},
\end{aligned}
$$

with $\hat{P}_{j}:=\hat{U}_{j}^{\text {ext }}-v_{j} \hat{U}^{\text {ext }}$ and $a_{a} \equiv a_{a}(0 \mathrm{PN})$, and

$$
{ }_{0} \bar{\Psi}=\hat{P}+A \partial_{\bar{t}} \hat{U}_{\text {ext }}+F^{p} \partial_{\bar{p}} \hat{U}_{\text {ext }}+\frac{1}{2} \dot{A}^{2}-\dot{A} v^{2}+\frac{1}{4} v^{4}+\dot{H}_{p} v^{p}-{ }_{0} \dot{\beta},
$$




$$
\begin{aligned}
{ }_{1} \bar{\Psi}_{a}= & \partial_{\bar{a}} \hat{P}+A \partial_{\bar{t} \bar{a}} \hat{U}_{\mathrm{ext}}+v_{a} \partial_{\bar{t}} \hat{U}_{\mathrm{ext}}+F^{p} \partial_{\bar{p} \bar{a}} \hat{U}_{\mathrm{ext}}+\left(\dot{A}-\frac{1}{2} v^{2}\right)\left[a_{a}(0 \mathrm{PN})-\partial_{\bar{a}} \hat{U}_{\mathrm{ext}}\right]-\frac{1}{2} v_{a} v^{p} \partial_{\bar{p}} \hat{U}_{\mathrm{ext}} \\
& +\epsilon_{p q a} R^{p} \partial^{\bar{q}} \hat{U}_{\mathrm{ext}}-a_{a}(1 \mathrm{PN})-\left[\ddot{A}-\frac{3}{2} v_{p} a^{p}(0 \mathrm{PN})\right] v_{a}-\epsilon_{a p q} v^{p} \dot{R}^{q}-{ }_{1} \dot{\beta}_{a} \\
{ }_{2} \bar{\Psi}_{a b}= & \partial_{\bar{a} \bar{b} \bar{P}} \hat{P}+A \partial_{\bar{t} \bar{a} \bar{b}} \hat{U}_{\mathrm{ext}}+2 v_{(a} \partial_{\bar{t} \bar{b})} \hat{U}_{\mathrm{ext}}+F^{p} \partial_{\bar{p} \bar{a} \bar{b}} \hat{U}_{\mathrm{ext}}-2\left(\dot{A}-\frac{1}{2} v^{2}\right) \partial_{\bar{a} \bar{b}} \hat{U}_{\mathrm{ext}}-v^{p} v_{(a} \partial_{\bar{b}) \bar{p}} \hat{U}_{\mathrm{ext}} \\
& +2 \epsilon_{p q(a} R^{p} \partial_{\bar{q} \bar{q}}^{\bar{q}} \hat{U}_{\mathrm{ext}}-2 a_{(a}(0 \mathrm{PN}) \partial_{\bar{b})} \hat{U}_{\mathrm{ext}}+\delta_{a b} a^{p}(0 \mathrm{PN}) \partial_{\bar{p}} \hat{U}_{\mathrm{ext}}+a_{a}(0 \mathrm{PN}) a_{b}(0 \mathrm{PN}) \\
& -2 v_{(a} \dot{a}_{b)}(0 \mathrm{PN})+\delta_{a b} v_{p} \dot{a}^{p}(0 \mathrm{PN})-{ }_{2} \dot{\beta}_{a b} \\
{ }_{3} \bar{\Psi}_{a b c}= & \partial_{\bar{a} \bar{b} \bar{c}} \hat{P}+A \partial_{\bar{t} \bar{a} \bar{b} \bar{c}} \hat{U}_{\mathrm{ext}}+3 v_{(a} \partial_{\bar{t} \overline{\bar{c}} \bar{c})} \hat{U}_{\mathrm{ext}}+F^{p} \partial_{\bar{p} \bar{a} \bar{b} \bar{c}} \hat{U}_{\mathrm{ext}}-3\left(\dot{A}-\frac{1}{2} v^{2}\right) \partial_{\bar{a} \bar{b} \bar{c}} \hat{U}_{\mathrm{ext}}-\frac{3}{2} v^{p} v_{(a} \partial_{\bar{b} \bar{c}) \bar{p}} \hat{U}_{\mathrm{ext}} \\
& +3 \epsilon_{p q(a} R^{p} \partial_{\bar{b} \bar{c})}^{\bar{q}} \hat{U}_{\mathrm{ext}}-6 a_{(a}(0 \mathrm{PN}) \partial_{\bar{b} \bar{c})} \hat{U}_{\mathrm{ext}}+3 \delta_{(a b} a^{p}(0 \mathrm{PN}) \partial_{\bar{c}) \bar{p}} \hat{U}_{\mathrm{ext}}-{ }_{3} \dot{\beta}_{a b c}, \\
{ }_{4} \bar{\Psi}_{a b c d}= & \partial_{\bar{a} \bar{b} \bar{c} \bar{d}} \hat{P}+A \partial_{\bar{t} \bar{a} \bar{b} \bar{c} \bar{U}} \hat{U}_{\mathrm{ext}}+4 v_{(a} \partial_{\bar{t} \bar{b} \bar{c} \bar{c})} \hat{U}_{\mathrm{ext}}+F^{p} \partial_{\bar{p} \bar{a} \bar{b} \bar{c} \bar{d}} \hat{U}_{\mathrm{ext}}-4\left(\dot{A}-\frac{1}{2} v^{2}\right) \partial_{\bar{a} \bar{b} \bar{c} \bar{d}} \hat{U}_{\mathrm{ext}}-2 v^{p} v_{(a} \partial_{\bar{b} \bar{c} \bar{d}) \bar{p}} \hat{U}_{\mathrm{ext}} \\
& +4 \epsilon_{p q(a} R^{p} \partial_{\bar{b} \bar{c} \bar{d})} \hat{U}_{\mathrm{ext}}-12 a_{(a}(0 \mathrm{PN}) \partial_{\bar{b} \bar{c} \bar{d})} \hat{U}_{\mathrm{ext}}+6 \delta_{(a b} a^{p}(0 \mathrm{PN}) \partial_{\bar{c} \bar{d} \bar{p}} \hat{U}_{\mathrm{ext}}-{ }_{4} \dot{\beta}_{a b c d},
\end{aligned}
$$

with $\hat{P}:=\hat{\Psi}_{\text {ext }}-4 v_{p} \hat{U}_{\text {ext }}^{p}+2 v^{2} \hat{U}_{\text {ext }}$. As stated previously, it is understood that all external potentials and their derivatives are evaluated at $\bar{x}^{a}=0$.

\section{Matching}

The potentials of Eq. 8.14) are the barycentric potentials transformed to the black-hole frame, and they must agree with the potentials of Eq. (7.7), obtained in the post-Newtonian expansion of the black-hole metric. A precise match between the expressions shall reveal the details of the coordinate transformation, the identity of the metric function $\mu(\bar{t})$, and the tidal moments.

The Newtonian potentials match at order $\bar{r}^{-1}$, and a match at order $\bar{r}^{0}$ implies

$$
\dot{A}=\hat{U}_{\text {ext }}+\frac{1}{2} v^{2}
$$

A match at order $\bar{r}^{1}$ further reveals that $a_{a}(0 \mathrm{PN})=\partial_{\bar{a}} \hat{U}^{\text {ext }}$. Examining now the post-Newtonian potentials $\bar{\Psi}$, a match at order $\bar{r}^{-3}$ implies that $F_{a}=0$, so that $H_{a}=A v_{a}$. A match at order $\bar{r}^{-1}$ further produces $\mu=\frac{3}{2} v^{2}-\hat{U}_{\text {ext }}$. A match at order $\bar{r}^{0}$ then yields

$$
{ }_{0} \dot{\beta}=\hat{\Psi}_{\mathrm{ext}}-4 v_{p} \hat{U}_{\mathrm{ext}}^{p}+\frac{1}{2} \hat{U}_{\mathrm{ext}}^{2}+\frac{5}{2} v^{2} \hat{U}_{\mathrm{ext}}+\frac{3}{8} v^{4}+A\left[\partial_{\bar{t}} \hat{U}_{\mathrm{ext}}+v_{p} a^{p}(0 \mathrm{PN})\right] .
$$

Turning next to the vector potentials $\bar{U}_{j}$, a match at order $\bar{r}^{0}$ reveals that

$$
{ }_{1} \beta_{a}=-4 \hat{U}_{a}^{\mathrm{ext}}+\left(3 \hat{U}_{\mathrm{ext}}+\frac{1}{2} v^{2}\right) v_{a}+A a_{a}(0 \mathrm{PN})-\epsilon_{a p q} v^{q} R^{q} .
$$

A match at order $\bar{r}^{1}$ produces

$$
4 \partial_{\bar{b}} \hat{P}_{a}+\frac{3}{2} v_{a} a_{b}(0 \mathrm{PN})+\frac{1}{2} a_{a}(0 \mathrm{PN}) v_{b}-\delta_{a b} \partial_{\bar{p}} \hat{U}_{\mathrm{ext}}^{p}-\epsilon_{a b p} \dot{R}^{p}+{ }_{2} \beta_{a b}=0
$$

after making use of the harmonic condition of Eq. 8.9p. Taking the symmetric part of this equation yields

$$
{ }_{2} \beta_{a b}=-4 \partial_{(\bar{a}} \hat{U}_{b)}^{\text {ext }}+2 v_{(a} a_{b)}(0 \mathrm{PN})+\delta_{a b} \partial_{\bar{p}} \hat{U}_{\mathrm{ext}}^{p},
$$

while taking the antisymmetric part implies

$$
\epsilon_{a b p} \dot{R}^{p}=-4 \partial_{[\bar{a}} \hat{U}_{b]}^{\mathrm{ext}}-3 v_{[a} a_{b]}(0 \mathrm{PN}) .
$$

Returning to $\bar{\Psi}$, we find that a match at order $\bar{r}^{1}$ requires

$$
a_{a}(1 \mathrm{PN})=\partial_{\bar{a}} \hat{\Psi}_{\mathrm{ext}}-4 v_{p} \partial_{\bar{a}} \hat{U}_{\mathrm{ext}}^{p}+4 \partial_{\bar{t}} \hat{U}_{a}^{\mathrm{ext}}+\left(v^{2}-4 \hat{U}_{\mathrm{ext}}\right) a_{a}(0 \mathrm{PN})-\left[3 \partial_{\bar{t}} \hat{U}_{\mathrm{ext}}+v_{p} a^{p}(0 \mathrm{PN})\right] v_{a} .
$$


At this stage the acceleration of the black hole in the barycentric frame, $a^{a}=a^{a}(0 \mathrm{PN})+a^{a}(1 \mathrm{PN})$, is determined, and some important details of the coordinate transformation are provided by Eqs. (8.18) and (8.23).

We next match the terms in the potentials that occur at order $\bar{r}^{2}$ and beyond. The Newtonian potential returns

$$
\overline{\mathcal{E}}_{a b}(0 \mathrm{PN})=-\partial_{\bar{a} \bar{b}} \hat{U}_{\mathrm{ext}}, \quad \overline{\mathcal{E}}_{a b c}(0 \mathrm{PN})=-\partial_{\bar{a} \bar{b} \bar{c}} \hat{U}_{\mathrm{ext}}, \quad 2 \overline{\mathcal{E}}_{a b c d}(0 \mathrm{PN})=-\partial_{\bar{a} \bar{b} \bar{c} \bar{d}} \hat{U}_{\mathrm{ext}},
$$

where (as stated previously) the hatted potentials are evaluated at $\bar{x}^{a}=0$ after differentiation.

From the vector potential at order $\bar{r}^{2}$ we get the matching condition

$$
-\frac{2}{3}\left(\epsilon_{j a}{ }^{p} \overline{\mathcal{B}}_{p b}+\epsilon_{j b}{ }^{p} \overline{\mathcal{B}}_{p a}\right)=4 \partial_{\bar{a} \bar{b}} \hat{P}_{j}+\delta_{j a} \dot{a}_{b}+\delta_{j b} \dot{a}_{a}-\delta_{a b} \dot{a}_{j}+{ }_{3} \beta_{j a b},
$$

in which $a_{a} \equiv a_{a}(0 \mathrm{PN})$ - the same shorthand will be employed in all equations below. To extract the consequences of this equation we decompose ${ }_{3} \beta_{j a b}$ into irreducible pieces according to Eq. (A1b), and the remaining terms of the right-hand side are decomposed according to Eq. A2). Equating all this with the left-hand side reveals that

$$
{ }_{3} \beta_{\langle a b c\rangle}=-4 \partial_{\langle\bar{a} \bar{b}}\left(\hat{U}_{c\rangle}^{\mathrm{ext}}-v_{c\rangle} \hat{U}^{\mathrm{ext}}\right), \quad{ }_{3} \beta_{a}=\dot{a}_{a},
$$

and

$$
\overline{\mathcal{B}}_{a b}=2 \epsilon_{(a}^{p q} \partial_{\bar{b}) \bar{p}}\left(\hat{U}_{q}^{\text {ext }}-v_{q} \hat{U}^{\text {ext }}\right) .
$$

To arrive at these results we made use of the harmonic condition of Eq. 8.9) and the field equations 8.10). At the next order we get

$$
-\frac{2}{3}\left(\epsilon_{j a}{ }^{p} \overline{\mathcal{B}}_{p b c}+\epsilon_{j c}{ }^{p} \overline{\mathcal{B}}_{p a b}+\epsilon_{j b}{ }^{p} \overline{\mathcal{B}}_{p c a}\right)+\frac{4}{3}\left(\delta_{j a} \dot{\overline{\mathcal{E}}}_{b c}+\delta_{j c} \dot{\overline{\mathcal{E}}}_{a b}+\delta_{j b} \dot{\overline{\mathcal{E}}}_{c a}\right)=4 \partial_{\bar{a} \bar{b} \bar{c}} \hat{P}_{j}+{ }_{4} \beta_{j a b c},
$$

and we decompose ${ }_{4} \beta_{j a b c}$ according to Eq. A1c, and $4 \partial_{\bar{a} \bar{b} \bar{c}} \hat{P}_{j}$ according to Eq. A4 . After simplifying the results with the harmonic condition, the field equations, and Eq. (8.25), we arrive at

$$
{ }_{4} \beta_{\langle a b c d\rangle}=-4 \partial_{\langle\bar{a} \bar{b} \bar{c}}\left(\hat{U}_{d\rangle}^{\text {ext }}-v_{d\rangle} \hat{U}^{\text {ext }}\right), \quad{ }_{4} \beta_{\langle a b\rangle}=\frac{8}{3} \dot{\overline{\mathcal{E}}}_{a b}, \quad{ }_{4} \beta=0,
$$

and

$$
\overline{\mathcal{B}}_{a b c}=\frac{3}{2} \epsilon_{(a}^{p q} \partial_{\bar{b} \bar{c}) \bar{p}}\left(\hat{U}_{q}^{\mathrm{ext}}-v_{q} \hat{U}^{\mathrm{ext}}\right) .
$$

Matching the vector potentials at order $\bar{r}^{4}$ produces

$$
-\frac{4}{3}\left(\epsilon_{j a}^{p} \overline{\mathcal{B}}_{p b c d}+\epsilon_{j d}{ }^{p} \overline{\mathcal{B}}_{p a b c}+\epsilon_{j c}{ }^{p} \overline{\mathcal{B}}_{p d a b}+\epsilon_{j b}{ }^{p} \overline{\mathcal{B}}_{p c d a}\right)+\left(\delta_{j a} \dot{\overline{\mathcal{E}}}_{b c d}+\delta_{j d} \dot{\overline{\mathcal{E}}}_{a b c}+\delta_{j c} \dot{\overline{\mathcal{E}}}_{d a b}+\delta_{j b} \dot{\overline{\mathcal{E}}}_{c d a}\right)=4 \partial_{\bar{a} \bar{b} \bar{c} \bar{d}} \hat{P}_{j}+{ }_{5} \beta_{j a b c d} .
$$

After decomposing ${ }_{5} \beta_{j a b c d}$ according to Eq. A1d, $4 \partial_{\bar{a} \bar{b} \bar{c} \bar{d}} \hat{P}_{j}$ according to Eq. A4, and simplifying, we arrive at

$$
{ }_{5} \beta_{\langle a b c d e\rangle}=-4 \partial_{\langle\bar{a} \bar{b} \bar{c} \bar{d}}\left(\hat{U}_{e\rangle}^{\mathrm{ext}}-v_{e\rangle} \hat{U}^{\mathrm{ext}}\right), \quad{ }_{5} \beta_{\langle a b c\rangle}=2 \dot{\overline{\mathcal{E}}}_{a b c}, \quad{ }_{5} \beta_{a}=0
$$

and

$$
\overline{\mathcal{B}}_{a b c d}=\frac{3}{5} \epsilon_{(a}^{p q} \partial_{\bar{b} \bar{c} \bar{d}) \bar{p}}\left(\hat{U}_{q}^{\mathrm{ext}}-v_{q} \hat{U}^{\mathrm{ext}}\right) .
$$

At this stage the gravitomagnetic tidal moments are all determined, as well as the details of the coordinate transformation contained in the function $\beta(\bar{t}, \overline{\boldsymbol{x}})$.

We finally return to the post-Newtonian potential. Matching at order $\bar{r}^{2}$ implies that

$$
\overline{\mathcal{E}}_{a b}(1 \mathrm{PN})=-\partial_{\bar{a} \bar{b}} \hat{P}-2 \hat{U}_{\mathrm{ext}} \overline{\mathcal{E}}_{a b}-v_{(a} \overline{\mathcal{E}}_{b) p} v^{p}+a_{a} a_{b}-\delta_{a b}\left(a^{2}+v_{p} \dot{a}^{p}\right)+{ }_{2} \dot{\beta}_{a b}+A \dot{\overline{\mathcal{E}}}_{a b}+2 \epsilon_{p q(a} R^{p} \overline{\mathcal{E}}_{b)}^{q},
$$

where $\hat{P}:=\hat{\Psi}_{\text {ext }}-4 v_{p} \hat{U}_{\text {ext }}^{p}+2 v^{2} \hat{U}_{\text {ext }}, a_{a} \equiv a_{a}(0 \mathrm{PN})$, and $\overline{\mathcal{E}}_{a b} \equiv \overline{\mathcal{E}}_{a b}(0 \mathrm{PN})$ on the right-hand side of the equation - a similar shorthand will be employed in all equations below. Making use of Eq. 8.22 and decomposing all tensors into irreducible pieces, we arrive at

$$
\overline{\mathcal{E}}_{a b}(1 \mathrm{PN})=-\partial_{\langle\bar{a} \bar{b}\rangle} \hat{P}-4 \partial_{\bar{t}\langle\bar{a}} \hat{U}_{b\rangle}^{\text {ext }}-2 \hat{U}_{\text {ext }} \overline{\mathcal{E}}_{a b}-v_{\langle a} \overline{\mathcal{E}}_{b\rangle p} v^{p}+3 a_{\langle a} a_{b\rangle}+2 v_{\langle a} \dot{a}_{b\rangle}+A \dot{\overline{\mathcal{E}}}_{a b}+2 \epsilon_{p q(a} R^{p} \overline{\mathcal{E}}_{b)}^{q} .
$$


At order $\bar{r}^{3}$ we get

$$
\overline{\mathcal{E}}_{a b c}(1 \mathrm{PN})=-\partial_{\bar{a} \bar{b} \bar{c}} \hat{P}+3 v_{(a} \dot{\overline{\mathcal{E}}}_{b c)}-3 \hat{U}_{\mathrm{ext}} \overline{\mathcal{E}}_{a b c}-\frac{3}{2} v_{(a} \overline{\mathcal{E}}_{b c) p} v^{p}-6 a_{(a} \overline{\mathcal{E}}_{b c)}+3 \delta_{(a b} \overline{\mathcal{E}}_{c) p} a^{p}+{ }_{3} \dot{\beta}_{a b c}+A \dot{\overline{\mathcal{E}}}_{a b c}+3 \epsilon_{p q(a} R^{p} \overline{\mathcal{E}}_{b c)}^{q},
$$

and substitution of Eq. (8.27) and decomposition into irreducible pieces yields

$$
\overline{\mathcal{E}}_{a b c}(1 \mathrm{PN})=-\partial_{\langle\bar{a} \bar{b} \bar{c}\rangle} \hat{P}-4 \partial_{\bar{t}\langle\bar{a} \bar{b}} \hat{U}_{c\rangle}^{\mathrm{ext}}-v_{\langle a} \dot{\overline{\mathcal{E}}}_{b c\rangle}-10 a_{\langle a} \overline{\mathcal{E}}_{b c\rangle}-3 \hat{U}_{\mathrm{ext}} \overline{\mathcal{E}}_{a b c}-\frac{3}{2} v_{\langle a} \overline{\mathcal{E}}_{b c\rangle p} v^{p}+A \dot{\overline{\mathcal{E}}}_{a b c}+3 \epsilon_{p q(a} R^{p} \overline{\mathcal{E}}_{b c)}^{q}
$$

At order $\bar{r}^{4}$ the matching condition is

$$
\begin{aligned}
& 2 \overline{\mathcal{E}}_{a b c d}(1 \mathrm{PN})+\frac{11}{21}\left(\delta_{a b} \ddot{\overline{\mathcal{E}}}_{c d}+\text { all symmetric permutations }\right)-10 \overline{\mathcal{E}}_{\langle a b} \overline{\mathcal{E}}_{c d\rangle}=-\partial_{\bar{a} \bar{b} \bar{c} \bar{d}} \hat{P}+4 v_{(a} \dot{\overline{\mathcal{E}}}_{b c d)}-8 \hat{U}_{\text {ext }} \overline{\mathcal{E}}_{a b c d} \\
& \quad-4 v_{(a} \overline{\mathcal{E}}_{b c d) p} v^{p}-12 a_{(a} \overline{\mathcal{E}}_{b c d)}+6 \delta_{(a b} \overline{\mathcal{E}}_{c d) p} a^{p}+{ }_{4} \dot{\beta}_{a b c d}+2 A \dot{\overline{\mathcal{E}}}_{a b c d}+8 \epsilon_{p q(a} R^{p} \overline{\mathcal{E}}_{b c d)}^{q},
\end{aligned}
$$

and this yields

$$
\begin{aligned}
2 \overline{\mathcal{E}}_{a b c d}(1 \mathrm{PN})= & -\partial_{\langle\bar{a} \bar{b} \bar{c} \bar{d}\rangle} \hat{P}-4 \partial_{\bar{t}\langle\bar{a} \bar{b} \bar{c}} \hat{U}_{d\rangle}^{\text {ext }}+10 \overline{\mathcal{E}}_{\langle a b} \overline{\mathcal{E}}_{c d\rangle}-16 a_{\langle a} \overline{\mathcal{E}}_{b c d\rangle} \\
& -8 \hat{U}_{\text {ext }} \overline{\mathcal{E}}_{a b c d}-4 v_{\langle a} \overline{\mathcal{E}}_{b c d\rangle p} v^{p}+2 A \dot{\overline{\mathcal{E}}}_{a b c d}+8 \epsilon_{p q(a} R^{p} \overline{\mathcal{E}}_{b c d)}^{q}
\end{aligned}
$$

after substituting Eq. (8.30) and decomposing all terms into irreducible pieces. At this stage the gravitoelectric tidal moments are all determined, and the matching procedure has come to a close.

\section{Barycentric tidal moments}

The tidal moments obtained in the preceding subsection are defined in the black-hole frame $\left(\bar{t}, \boldsymbol{x}^{a}\right)$. For our purposes in Sec. IX, it is convenient to follow Racine and Flanagan [35] and introduce barycentric versions of these moments. We do so with the transformations

$$
\begin{aligned}
\mathcal{E}_{a b}(t) & :=\mathcal{M}_{a}^{j}(\bar{t}) \mathcal{M}_{b}{ }^{k}(\bar{t}) \overline{\mathcal{E}}_{j k}(\bar{t}) \\
\mathcal{E}_{a b c}(t) & :=\mathcal{M}_{a}^{j}(\bar{t}) \mathcal{M}_{b}{ }^{k}(\bar{t}) \mathcal{M}_{c}^{m}(\bar{t}) \overline{\mathcal{E}}_{j k m}(\bar{t}) \\
\mathcal{E}_{a b c d}(t) & :=\mathcal{M}_{a}^{j}(\bar{t}) \mathcal{M}_{b}{ }^{k}(\bar{t}) \mathcal{M}_{c}^{m}(\bar{t}) \mathcal{M}_{d}^{n}(\bar{t}) \overline{\mathcal{E}}_{j k m n}(\bar{t}),
\end{aligned}
$$

as well as similar ones relating $\mathcal{B}_{a b \ldots}(t)$ to $\overline{\mathcal{B}}_{a b \ldots}(\bar{t})$. The transformation matrix is defined by

$$
\mathcal{M}_{a j}(\bar{t}):=\delta_{a j}+\epsilon_{a j p} R^{p}(\bar{t})+2 \mathrm{PN}
$$

with $R^{p}$ determined by Eq. 8.23. Because the tidal moments are tensors defined at $\bar{x}^{a}=0$, the relation between the time coordinates is given by

$$
t=\bar{t}+A(\bar{t})+2 \mathrm{PN}
$$

with $A$ determined by Eq. 8.18.

We apply Eq. 8.41 to the tidal moments obtained previously, and express the results in terms of the original potentials $U, U_{j}$, and $\Psi$ (instead of the hatted ones). Recalling the differentiation rule of Eq. (8.8), we find that the barycentric version of the gravitoelectric tidal moments are given by

$$
\begin{aligned}
\mathcal{E}_{a b}= & \mathcal{E}_{a b}(0 \mathrm{PN})+\mathcal{E}_{a b}(1 \mathrm{PN})+2 \mathrm{PN} \\
\mathcal{E}_{a b}(0 \mathrm{PN})= & -\partial_{a b} U_{\text {ext }} \\
\mathcal{E}_{a b}(1 \mathrm{PN})= & -\partial_{\langle a b\rangle} \Psi_{\mathrm{ext}}-4 \partial_{t\langle a} U_{b\rangle}^{\mathrm{ext}}+4\left(\partial_{\langle a b\rangle} U_{p}^{\mathrm{ext}}-\partial_{p\langle a} U_{b\rangle}^{\mathrm{ext}}\right) v^{p}+2\left(v^{2}-U_{\mathrm{ext}}\right) \mathcal{E}_{a b} \\
& -v_{\langle a} \mathcal{E}_{b\rangle p} v^{p}+3 a_{\langle a} a_{b\rangle}+2 v_{\langle a} \dot{a}_{b\rangle} \\
\mathcal{E}_{a b c}= & \mathcal{E}_{a b c}(0 \mathrm{PN})+\mathcal{E}_{a b c}(1 \mathrm{PN})+2 \mathrm{PN} \\
\mathcal{E}_{a b c}(0 \mathrm{PN})= & -\partial_{a b c} U_{\mathrm{ext}},
\end{aligned}
$$




$$
\begin{aligned}
\mathcal{E}_{a b c}(1 \mathrm{PN})= & -\partial_{\langle a b c\rangle} \Psi_{\text {ext }}-4 \partial_{t\langle a b} U_{c\rangle}^{\text {ext }}+4\left(\partial_{\langle a b c\rangle} U_{p}^{\text {ext }}-\partial_{p\langle a b} U_{c\rangle}^{\text {ext }}\right) v^{p}+\left(2 v^{2}-3 U_{\text {ext }}\right) \mathcal{E}_{a b c} \\
& -\frac{3}{2} v_{\langle a} \mathcal{E}_{b c\rangle p} v^{p}-v_{\langle a} \dot{\mathcal{E}}_{b c\rangle}-10 a_{\langle a} \mathcal{E}_{b c\rangle},
\end{aligned}
$$

and

$$
\begin{aligned}
\mathcal{E}_{a b c d}= & \mathcal{E}_{a b c d}(0 \mathrm{PN})+\mathcal{E}_{a b c d}(1 \mathrm{PN})+2 \mathrm{PN}, \\
2 \mathcal{E}_{a b c d}(0 \mathrm{PN})= & -\partial_{a b c d} U_{\mathrm{ext}}, \\
2 \mathcal{E}_{a b c d}(1 \mathrm{PN})= & -\partial_{\langle a b c d\rangle} \Psi_{\mathrm{ext}}-4 \partial_{t\langle a b c} U_{d\rangle}^{\mathrm{ext}}+4\left(\partial_{\langle a b c d\rangle} U_{p}^{\mathrm{ext}}-\partial_{p\langle a b c} U_{d\rangle}^{\mathrm{ext}}\right) v^{p}+4\left(v^{2}-2 U_{\mathrm{ext}}\right) \mathcal{E}_{a b c d} \\
& -4 v_{\langle a} \mathcal{E}_{b c d\rangle} v^{p}-16 a_{\langle a} \mathcal{E}_{b c d\rangle}+10 \mathcal{E}_{\langle a b} \mathcal{E}_{c d\rangle} .
\end{aligned}
$$

The external potentials are evaluated at $x^{a}=r^{a}(t)$ after differentiation, and it is understood that in the expressions for the post-Newtonian terms, $a_{a} \equiv a_{a}(0 \mathrm{PN})=\partial_{a} U_{\text {ext }}, \mathcal{E}_{a b} \equiv \mathcal{E}_{a b}(0 \mathrm{PN})$, and so on.

Because the gravitomagnetic moments are quantities of $1 \mathrm{PN}$ order, the transformations analogous to those of Eq. 8.41 have a trivial effect on them. The barycentric version of these moments are therefore given by

$$
\begin{aligned}
\mathcal{B}_{a b} & =2 \epsilon_{(a}^{p q} \partial_{b) p}\left(U_{q}^{\mathrm{ext}}-v_{q} U^{\mathrm{ext}}\right), \\
\mathcal{B}_{a b c} & =\frac{3}{2} \epsilon_{(a}^{p q} \partial_{b c) p}\left(U_{q}^{\mathrm{ext}}-v_{q} U^{\mathrm{ext}}\right), \\
\mathcal{B}_{a b c d} & =\frac{3}{5} \epsilon_{(a}^{p q} \partial_{b c d) p}\left(U_{q}^{\mathrm{ext}}-v_{q} U^{\mathrm{ext}}\right) .
\end{aligned}
$$

Here also the external potentials are evaluated at $x^{a}=r^{a}(t)$ after differentiation.

\section{TIDAL MOMENTS FOR A TWO-BODY SYSTEM}

The tidal moments obtained in Sec. VIII are expressed in terms of generic external potentials that could describe any post-Newtonian spacetime. In this section we specialize them to the specific case in which the black hole is a member of a two-body system.

\section{A. External potentials}

We take the spacetime to contain a body of mass $M_{2}$ at position $\boldsymbol{r}_{2}(t)$ in addition to the black hole. We let $\boldsymbol{v}_{2}(t):=d \boldsymbol{r}_{2} / d t$ and $\boldsymbol{a}_{2}(t):=d \boldsymbol{v}_{2} / d t$. We refine the notation employed in Sec. VIII the black hole's mass shall again be denoted $M_{1}$ instead of $M$, and its position in the barycentric frame shall be $\boldsymbol{r}_{1}(t)$ instead of $\boldsymbol{r}$; we also set $\boldsymbol{v}_{1}(t):=d \boldsymbol{r}_{1} / d t$ (previously $\boldsymbol{v}$ ) and $\boldsymbol{a}_{1}(t):=d \boldsymbol{v}_{1} / d t$ (previously $\boldsymbol{a}$ ).

We take the external body to be another post-Newtonian monopole, and write the external potentials as

$$
U_{\mathrm{ext}}=\frac{M_{2}}{s}, \quad U_{\mathrm{ext}}^{j}=\frac{M_{2} v_{2}^{j}}{s}, \quad \psi_{\mathrm{ext}}=\frac{M_{2} \mu_{2}}{s}, \quad X_{\mathrm{ext}}=M_{2} s,
$$

where $s$ now stands for $\left|\boldsymbol{x}-\boldsymbol{r}_{2}\right|$, the Euclidean distance between the field point $\boldsymbol{x}$ and the external body. We recall that $\Psi_{\text {ext }}=\psi_{\text {ext }}+\frac{1}{2} \partial_{t t} X_{\text {ext }}$. According to our findings in Sec. VIIIC, the post-Newtonian correction to the mass parameter is given by $\mu_{2}=\frac{3}{2} v_{2}^{2}-M_{1} / b$, where $b$ is the inter-body distance.

We let $\boldsymbol{b}:=\boldsymbol{r}_{1}-\boldsymbol{r}_{2}$ be the separation between bodies, $b:=|\boldsymbol{b}|$, and $\boldsymbol{n}:=\boldsymbol{b} / b$ is a unit vector directed from the external body to the black hole. The relative velocity is $\boldsymbol{v}:=\boldsymbol{v}_{1}-\boldsymbol{v}_{2}$, and $\dot{b}=\boldsymbol{v} \cdot \boldsymbol{n}$ is its radial component. The post-Newtonian equations of motion imply that $\boldsymbol{a}_{1}=-\left(M_{2} / b^{2}\right) \boldsymbol{n}+1 \mathrm{PN}$ and $\boldsymbol{a}_{2}=\left(M_{1} / b^{2}\right) \boldsymbol{n}+1 \mathrm{PN}$. They also imply that $\boldsymbol{v}_{1}=q_{2} \boldsymbol{v}+1 \mathrm{PN}$ and $\boldsymbol{v}_{2}=-q_{1} \boldsymbol{v}+1 \mathrm{PN}$, where

$$
q_{1}:=\frac{M_{1}}{M_{1}+M_{2}}, \quad q_{2}:=\frac{M_{2}}{M_{1}+M_{2}}
$$

are the mass ratios, constrained by the identity $q_{1}+q_{2}=1$.

The various derivatives of the external potentials are evaluated with the help of results collected in Appendix B. The position $\boldsymbol{x}$ is set equal to $\boldsymbol{r}_{1}$ after differentiation, and in this limit the vector $\boldsymbol{s}:=\boldsymbol{x}-\boldsymbol{r}_{2}$ becomes $\boldsymbol{b}$; similarly, $\hat{\boldsymbol{s}} \rightarrow \boldsymbol{n}$ and $s \rightarrow b$. The equations of motion are used to eliminate the accelerations $\boldsymbol{a}_{1}$ and $\boldsymbol{a}_{2}$ from all expressions, and to express the velocities $\boldsymbol{v}_{1}$ and $\boldsymbol{v}_{2}$ in terms of the relative velocity $\boldsymbol{v}$. 


\section{B. Barycentric tidal moments}

A long but straightforward calculation returns

$$
\begin{gathered}
\mathcal{E}_{a b}=\mathcal{E}_{a b}(0 \mathrm{PN})+\mathcal{E}_{a b}(1 \mathrm{PN})+2 \mathrm{PN} \\
\mathcal{E}_{a b}(\mathrm{PPN})=-3 \frac{M_{2}}{b^{3}} n_{\langle a b\rangle}, \\
\mathcal{E}_{a b}(1 \mathrm{PN})=-3 \frac{M_{2}}{b^{3}}\left\{\left[2 v^{2}-\frac{5}{2} q_{1}^{2} \dot{b}^{2}-\frac{1}{2}\left(6-q_{1}\right) \frac{M}{b}\right] n_{\langle a b\rangle}-\left(3-q_{1}^{2}\right) \dot{b} v_{\langle a} n_{b\rangle}+v_{\langle a} v_{b\rangle}\right\}, \\
\mathcal{E}_{a b c}(1 \mathrm{PN})=15 \frac{M_{2}}{b^{4}}\left\{\left[2 v^{2}-\frac{7}{2} q_{1}^{2} \dot{b}^{2}-\left(5-3 q_{1}\right) \frac{M}{b}\right] n_{\langle a b c\rangle}-\frac{1}{2}\left(5-3 q_{1}^{2}\right) \dot{b} v_{\langle a} n_{b} n_{c\rangle}+v_{\langle a} v_{b} n_{c\rangle}\right\} \\
\mathcal{E}_{a b c}=\mathcal{E}_{a b c}(0 \mathrm{PN})+\mathcal{E}_{a b c}(1 \mathrm{PN})+2 \mathrm{PN}, \\
\mathcal{E}_{a b c d}=15 \frac{M_{2}}{b^{4}} n_{\langle a b c\rangle}, \\
\mathcal{E}_{a b c d}(0 \mathrm{PN})+\mathcal{E}_{a b c d}(1 \mathrm{PN})+2 \mathrm{PN}, \\
\mathcal{E}_{a b c d}(1 \mathrm{PN})=-\frac{105}{2} \frac{M_{2}}{b^{5}} n_{\langle a b c d\rangle}, \\
\frac{15}{2} \frac{M_{2}}{b^{5}}\left\{\left[14 v^{2}-\frac{63}{2} q_{1}^{2} \dot{b}^{2}-\frac{25}{2}\left(4-3 q_{1}\right) \frac{M}{b}\right] n_{\langle a b c d\rangle}-14\left(1-q_{1}^{2}\right) \dot{b} v_{\langle a} n_{b} n_{c} n_{d\rangle}+6 v_{\langle a} v_{b} n_{c} n_{d\rangle}\right\}
\end{gathered}
$$

for the barycentric version of the gravitoelectric tidal moments, where $n_{\langle a b \cdots c\rangle}$ is shorthand for $n_{\langle a} n_{b} \cdots n_{c\rangle}$. The gravitomagnetic moments are given by

$$
\begin{aligned}
\mathcal{B}_{a b} & =-6 \frac{M_{2}}{b^{3}} \epsilon_{p q(a} n_{b)} n^{p} v^{q} \\
\mathcal{B}_{a b c} & =\frac{45}{2} \frac{M_{2}}{b^{4}} \epsilon_{p q(a}\left(n_{b} n_{c)}-\frac{1}{5} \delta_{b c)}\right) n^{p} v^{q}, \\
\mathcal{B}_{a b c d} & =-63 \frac{M_{2}}{b^{5}} \epsilon_{p q(a}\left(n_{b} n_{c} n_{d)}-\frac{3}{7} \delta_{b c} n_{d)}\right) n^{p} v^{q} .
\end{aligned}
$$

Alternative expressions are obtained if the relative velocity vector is decomposed according to

$$
\boldsymbol{v}=\dot{b} \boldsymbol{n}+v_{\perp} \boldsymbol{\lambda}
$$

in terms of radial and perpendicular components; $\boldsymbol{\lambda}$ is a unit vector orthogonal to $\boldsymbol{n}$. We recall that the postNewtonian motion takes place in a fixed orbital plane, with a vanishing normal component for the velocity. The tidal moments become

$$
\begin{aligned}
\mathcal{E}_{a b}(1 \mathrm{PN})= & 3 \frac{M_{2}}{b^{3}}\left\{\left[\frac{3}{2} q_{1}^{2} \dot{b}^{2}-2 v_{\perp}^{2}+\frac{1}{2}\left(6-q_{1}\right) \frac{M}{b}\right] n_{\langle a b\rangle}+\left(1-q_{1}^{2}\right) \dot{b} v_{\perp} \lambda_{\langle a} n_{b\rangle}-v_{\perp}^{2} \lambda_{\langle a} \lambda_{b\rangle}\right\}, \\
\mathcal{E}_{a b c}(1 \mathrm{PN})= & 15 \frac{M_{2}}{b^{4}}\left\{\left[\frac{1}{2}\left(1-4 q_{1}^{2}\right) \dot{b}^{2}+2 v_{\perp}^{2}-\left(5-3 q_{1}\right) \frac{M}{b}\right] n_{\langle a b c\rangle}-\frac{1}{2}\left(1-3 q_{1}^{2}\right) \dot{b} v_{\perp} \lambda_{\langle a} n_{b} n_{c\rangle}+v_{\perp}^{2} \lambda_{\langle a} \lambda_{b} n_{c\rangle}\right\} \\
\mathcal{E}_{a b c d}(1 \mathrm{PN})= & -\frac{15}{2} \frac{M_{2}}{b^{5}}\left\{\left[\frac{1}{2}\left(12-35 q_{1}^{2}\right) \dot{b}^{2}+14 v_{\perp}^{2}-\frac{25}{2}\left(4-3 q_{1}\right) \frac{M}{b}\right] n_{\langle a b c d\rangle}\right. \\
& \left.-2\left(1-7 q_{1}^{2}\right) \dot{b}_{\perp} \lambda_{\langle a} n_{b} n_{c} n_{d\rangle}+6 v_{\perp}^{2} \lambda_{\langle a} \lambda_{b} n_{c} n_{d\rangle}\right\}
\end{aligned}
$$

and

$$
\mathcal{B}_{a b}=-6 \frac{M_{2} v_{\perp}}{b^{3}} \epsilon_{p q(a} n_{b)} n^{p} \lambda^{q}
$$




$$
\begin{aligned}
\mathcal{B}_{a b c} & =\frac{45}{2} \frac{M_{2} v_{\perp}}{b^{4}} \epsilon_{p q(a}\left(n_{b} n_{c)}-\frac{1}{5} \delta_{b c)}\right) n^{p} \lambda^{q}, \\
\mathcal{B}_{a b c d} & =-63 \frac{M_{2} v_{\perp}}{b^{5}} \epsilon_{p q(a}\left(n_{b} n_{c} n_{d)}-\frac{3}{7} \delta_{b c} n_{d)}\right) n^{p} \lambda^{q} .
\end{aligned}
$$

The tidal moments in the black-hole frame are obtained by inverting the transformation of Eqs. 8.41), (8.42), and (8.43). Making the relevant substitutions in Eq. 8.18), we find that $A$ is determined by

$$
\frac{d A}{d t}=\frac{1}{2}\left(1-q_{1}\right)^{2}\left(\dot{b}^{2}+v_{\perp}^{2}\right)+\left(1-q_{1}\right) \frac{M}{b},
$$

and

$$
\frac{d R_{a}}{d t}=-\frac{1}{2}\left(1-q_{1}\right)\left(3+q_{1}\right) \frac{M v_{\perp}}{b^{2}} \epsilon_{a b c} n^{b} \lambda^{c}
$$

follows from Eq. 8.23.

\section{Circular motion}

Setting $\dot{b}=0$ specializes the motion to a circular orbit with $b=$ constant. The equations of motion imply (see, for example, Sec. 10.1.2 of Ref. 34])

$$
V \equiv v_{\perp}=\sqrt{\frac{M}{b}}\left[1-\frac{1}{2}\left(3-q_{1} q_{2}\right) \frac{M}{b}+2 \mathrm{PN}\right],
$$

and this equation reveals that $M / b=V^{2}+1 \mathrm{PN}$. Making the substitutions within Eqs. 9.8 produces

$$
\begin{aligned}
\mathcal{E}_{a b}(1 \mathrm{PN}) & =3 \frac{M_{2} V^{2}}{b^{3}}\left[\frac{1}{2}\left(2-q_{1}\right) n_{\langle a b\rangle}-\lambda_{\langle a} \lambda_{b\rangle}\right], \\
\mathcal{E}_{a b c}(1 \mathrm{PN}) & =-15 \frac{M_{2} V^{2}}{b^{4}}\left[3\left(1-q_{1}\right) n_{\langle a b c\rangle}-\lambda_{\langle a} \lambda_{b} n_{c\rangle}\right], \\
\mathcal{E}_{a b c d}(1 \mathrm{PN}) & =\frac{15}{2} \frac{M_{2} V^{2}}{b^{5}}\left[\frac{1}{2}\left(72-75 q_{1}\right) n_{\langle a b c d\rangle}-6 \lambda_{\langle a} \lambda_{b} n_{c} n_{d\rangle}\right] .
\end{aligned}
$$

The equations (9.9) stay unchanged.

In the case of a circular orbit the basis vectors can be given the explicit representation

$$
\boldsymbol{n}=[\cos (\omega t), \sin (\omega t), 0], \quad \boldsymbol{\lambda}=[-\sin (\omega t), \cos (\omega t), 0],
$$

where

$$
\omega:=\frac{V}{b}=\sqrt{\frac{M}{b^{3}}}\left[1-\frac{1}{2}\left(3-q_{1} q_{2}\right) \frac{M}{b}+2 \mathrm{PN}\right]
$$

is the orbital angular velocity in the barycentric frame. It is useful to complete the vector basis with

$$
\ell:=[0,0,1]
$$

a unit vector normal to the orbital plane.

Equations 9.10 and 9.11 become

$$
\frac{d A}{d t}=\frac{1}{2}\left(1-q_{1}\right)\left(3-q_{1}\right) V^{2}, \quad \frac{d R^{a}}{d t}=-\frac{1}{2}\left(1-q_{1}\right)\left(3+q_{1}\right) \sqrt{\frac{M}{b^{3}}} V^{2} \ell^{a}
$$

in the case of circular motion. The equations integrate to

$$
A=k t, \quad k=\frac{1}{2}\left(1-q_{1}\right)\left(3-q_{1}\right) V^{2}
$$

and

$$
R^{a}=-\Omega t \ell^{a}, \quad \Omega:=\frac{1}{2}\left(1-q_{1}\right)\left(3+q_{1}\right) \sqrt{\frac{M}{b^{3}}} V^{2},
$$

with $\Omega$ denoting the precessional angular velocity of the black-hole frame relative to the barycentric frame. 


\section{Transformation to the black-hole frame}

The transformation from the barycentric frame of the post-Newtonian spacetime to the black hole's moving frame is effected by inverting Eqs. 8.41), 8.42), and 8.43). Under the inverse transformation, a vector $p_{a}(t)$ defined in the barycentric frame becomes

$$
\bar{p}_{a}(\bar{t})=\left[\mathcal{M}^{-1}(t)\right]_{a}^{j} p_{j}(t)
$$

in the black-hole frame, with

$$
\left[\mathcal{M}^{-1}(t)\right]_{a j}=\delta_{a j}-\epsilon_{a j p} R^{p}(t)+2 \mathrm{PN}
$$

and $\bar{t}=t-A(t)+2 \mathrm{PN}$. In the case of circular motion, Eqs. 9.18$)$ and $(9.19)$ imply that the transformation takes the form of

$$
\overline{\boldsymbol{p}}=\boldsymbol{p}-(\Omega t) \boldsymbol{\ell} \times \boldsymbol{p}
$$

with $t=(1+k) \bar{t}$ inserted onto the right-hand side. Applying this rule to our vectorial basis yields

$$
\overline{\boldsymbol{n}}=\boldsymbol{n}-(\Omega t) \boldsymbol{\lambda}, \quad \overline{\boldsymbol{\lambda}}=\boldsymbol{\lambda}+(\Omega t) \boldsymbol{n}, \quad \overline{\boldsymbol{\ell}}=\boldsymbol{\ell} .
$$

With the representation of Eqs. (9.14) and (9.16), and with $\Omega$ recognized as a post-Newtonian correction to the angular velocity $\omega$, we have that

$$
\overline{\boldsymbol{n}}=[\cos (\bar{\omega} \bar{t}), \sin (\bar{\omega} \bar{t}), 0], \quad \bar{\lambda}=[-\sin (\bar{\omega} \bar{t}), \cos (\bar{\omega} \bar{t}), 0], \quad \bar{\ell}=[0,0,1],
$$

where $\bar{\omega}=(1+k) \omega-\Omega$, or

$$
\bar{\omega}=\sqrt{\frac{M}{b^{3}}}\left[1-\frac{1}{2}\left(3+q_{1} q_{2}\right) V^{2}+2 \mathrm{PN}\right] .
$$

This is the angular frequency of the tidal field as measured in the black-hole frame. It differs from $\omega$, the orbital angular velocity in the barycentric frame, because of the mismatch in the time coordinates (measured by $k$ ), and also because of the relative precession of the two frames (measured by $\Omega$ ).

The tidal moments in the black-hole frame are obtained directly from the barycentric moments by replacing $\boldsymbol{n}$ with $\overline{\boldsymbol{n}}$, and $\boldsymbol{\lambda}$ with $\overline{\boldsymbol{\lambda}}$. In this transcription, however, we shall also take the opportunity to modify our convention for the basis vectors. We recall that $\boldsymbol{n}$ is proportional to $\boldsymbol{r}_{1}-\boldsymbol{r}_{2}$, and is therefore directed from the external body to the black hole. In the black-hole frame it is convenient to reverse this direction, and we therefore let $\overline{\boldsymbol{n}} \rightarrow-\overline{\boldsymbol{n}}$ in our expressions for the tidal moments. Similarly, we recall $\boldsymbol{\lambda}$ is proportional to $\boldsymbol{v}_{1}-\boldsymbol{v}_{2}$, and choose to reverse this direction as well by letting $\bar{\lambda} \rightarrow-\bar{\lambda}$.

With these changes accounted for, we find that the tidal moments in the black-hole frame are given by

$$
\begin{aligned}
\overline{\mathcal{E}}_{a b} & =\overline{\mathcal{E}}_{a b}(\mathrm{OPN})+\overline{\mathcal{E}}_{a b}(1 \mathrm{PN})+2 \mathrm{PN} \\
\overline{\mathcal{E}}_{a b}(0 \mathrm{PN}) & =-3 \frac{M_{2}}{b^{3}} \bar{n}_{\langle a b\rangle}, \\
\overline{\mathcal{E}}_{a b}(1 \mathrm{PN}) & =3 \frac{M_{2} V^{2}}{b^{3}}\left[\frac{1}{2}\left(2-q_{1}\right) \bar{n}_{\langle a b\rangle}-\bar{\lambda}_{\langle a} \bar{\lambda}_{b\rangle}\right] \\
\overline{\mathcal{E}}_{a b c} & =\overline{\mathcal{E}}_{a b c}(0 \mathrm{PN})+\overline{\mathcal{E}}_{a b c}(1 \mathrm{PN})+2 \mathrm{PN} \\
\overline{\mathcal{E}}_{a b c}(0 \mathrm{PN}) & =-15 \frac{M_{2}}{b^{4}} \bar{n}_{\langle a b c\rangle}, \\
\overline{\mathcal{E}}_{a b c}(1 \mathrm{PN}) & =15 \frac{M_{2} V^{2}}{b^{4}}\left[3\left(1-q_{1}\right) \bar{n}_{\langle a b c\rangle}-\bar{\lambda}_{\langle a} \bar{\lambda}_{b} \bar{n}_{c\rangle}\right]
\end{aligned}
$$




$$
\begin{aligned}
\overline{\mathcal{E}}_{a b c d} & =\overline{\mathcal{E}}_{a b c d}(0 \mathrm{PN})+\overline{\mathcal{E}}_{a b c d}(1 \mathrm{PN})+2 \mathrm{PN}, \\
\overline{\mathcal{E}}_{a b c d}(0 \mathrm{PN}) & =-\frac{105}{2} \frac{M_{2}}{b^{5}} \bar{n}_{\langle a b c d\rangle}, \\
\overline{\mathcal{E}}_{a b c d}(1 \mathrm{PN}) & =\frac{15}{2} \frac{M_{2} V^{2}}{b^{5}}\left[\frac{1}{2}\left(72-75 q_{1}\right) \bar{n}_{\langle a b c d\rangle}-6 \bar{\lambda}_{\langle a} \bar{\lambda}_{b} \bar{n}_{c} \bar{n}_{d\rangle}\right] .
\end{aligned}
$$

and

$$
\begin{aligned}
\overline{\mathcal{B}}_{a b} & =6 \frac{M_{2} V}{b^{3}} \epsilon_{p q(a} \bar{n}_{b)} \bar{n}^{p} \bar{\lambda}^{q}, \\
\overline{\mathcal{B}}_{a b c} & =\frac{45}{2} \frac{M_{2} V}{b^{4}} \epsilon_{p q(a}\left(\bar{n}_{b} \bar{n}_{c)}-\frac{1}{5} \delta_{b c)}\right) \bar{n}^{p} \bar{\lambda}^{q}, \\
\overline{\mathcal{B}}_{a b c d} & =63 \frac{M_{2} V}{b^{5}} \epsilon_{p q(a}\left(\bar{n}_{b} \bar{n}_{c} \bar{n}_{d)}-\frac{3}{7} \delta_{b c} \bar{n}_{d)}\right) \bar{n}^{p} \bar{\lambda}^{q} .
\end{aligned}
$$

\section{E. Harmonic components of the tidal moments}

The harmonic components of the tidal moments are defined in Tables III and IV Making use of Eqs. (9.26), (9.27), 9.28), and (9.29), we find that in the case of circular motion, the nonvanishing components are

$$
\begin{aligned}
& \overline{\mathcal{E}}_{0}^{\mathrm{q}}=-\frac{1}{2} \frac{M_{2}}{b^{3}}\left[1+\frac{1}{2} q_{1} V^{2}+2 \mathrm{PN}\right], \\
& \overline{\mathcal{E}}_{2 c}^{\mathrm{q}}=-\frac{3}{2} \frac{M_{2}}{b^{3}}\left[1+\frac{1}{2}\left(q_{1}-4\right) V^{2}+2 \mathrm{PN}\right] \cos (2 \bar{\omega} \bar{t}), \\
& \overline{\mathcal{E}}_{2 s}^{\mathrm{q}}=-\frac{3}{2} \frac{M_{2}}{b^{3}}\left[1+\frac{1}{2}\left(q_{1}-4\right) V^{2}+2 \mathrm{PN}\right] \sin (2 \bar{\omega} \bar{t}), \\
& \overline{\mathcal{E}}_{1 c}^{\circ}=-\frac{3}{2} \frac{M_{2}}{b^{4}}\left[1+\frac{1}{3}\left(9 q_{1}-8\right) V^{2}+2 \mathrm{PN}\right] \cos (\bar{\omega} \bar{t}), \\
& \overline{\mathcal{E}}_{1 s}^{\circ}=-\frac{3}{2} \frac{M_{2}}{b^{4}}\left[1+\frac{1}{3}\left(9 q_{1}-8\right) V^{2}+2 \mathrm{PN}\right] \sin (\bar{\omega} \bar{t}), \\
& \overline{\mathcal{E}}_{3 c}^{\circ}=-\frac{15}{4} \frac{M_{2}}{b^{4}}\left[1+\left(3 q_{1}-4\right) V^{2}+2 \mathrm{PN}\right] \cos (3 \bar{\omega} \bar{t}), \\
& \overline{\mathcal{E}}_{3 s}^{\circ}=-\frac{15}{4} \frac{M_{2}}{b^{4}}\left[1+\left(3 q_{1}-4\right) V^{2}+2 \mathrm{PN}\right] \sin (3 \bar{\omega} \bar{t}), \\
& \overline{\mathcal{E}}_{0}^{\mathrm{h}}=-\frac{9}{4} \frac{M_{2}}{b^{5}}\left[1+\frac{1}{14}\left(75 q_{1}-68\right) V^{2}+2 \mathrm{PN}\right] \\
& \overline{\mathcal{E}}_{2 c}^{\mathrm{h}}=-\frac{15}{2} \frac{M_{2}}{b^{5}}\left[1+\frac{3}{14}\left(25 q_{1}-24\right) V^{2}+2 \mathrm{PN}\right] \cos (2 \bar{\omega} \bar{t}), \\
& \overline{\mathcal{E}}_{2 s}^{\mathrm{h}}=-\frac{15}{2} \frac{M_{2}}{b^{5}}\left[1+\frac{3}{14}\left(25 q_{1}-24\right) V^{2}+2 \mathrm{PN}\right] \sin (2 \bar{\omega} \bar{t}), \\
& \overline{\mathcal{E}}_{4 c}^{\mathrm{h}}=-\frac{105}{8} \frac{M_{2}}{b^{5}}\left[1+\frac{3}{14}\left(25 q_{1}-28\right) V^{2}+2 \mathrm{PN}\right] \cos (4 \bar{\omega} \bar{t}), \\
& \overline{\mathcal{E}}^{5}\left[1+\frac{3}{14}\left(25 q_{1}-28\right) V^{2}+2 \mathrm{PN}\right] \sin (4 \bar{\omega} \bar{t}),
\end{aligned}
$$

and

$$
\overline{\mathcal{B}}_{1 c}^{\mathrm{q}}=3 \frac{M_{2} V}{b^{3}} \cos (\bar{\omega} \bar{t}),
$$




$$
\begin{aligned}
\overline{\mathcal{B}}_{1 s}^{\mathrm{q}} & =3 \frac{M_{2} V}{b^{3}} \sin (\bar{\omega} \bar{t}), \\
\overline{\mathcal{B}}_{0}^{\circ} & =3 \frac{M_{2} V}{b^{4}}, \\
\overline{\mathcal{B}}_{2 c}^{\circ} & =5 \frac{M_{2} V}{b^{4}} \cos (2 \bar{\omega} \bar{t}), \\
\overline{\mathcal{B}}_{2 s}^{\circ} & =5 \frac{M_{2} V}{b^{4}} \sin (2 \bar{\omega} \bar{t}), \\
\overline{\mathcal{B}}_{1 c}^{\mathrm{h}} & =\frac{45}{4} \frac{M_{2} V}{b^{5}} \cos (\bar{\omega} \bar{t}), \\
\overline{\mathcal{B}}_{1 s}^{\mathrm{h}} & =\frac{45}{4} \frac{M_{2} V}{b^{5}} \sin (\bar{\omega} \bar{t}), \\
\overline{\mathcal{B}}_{3 c}^{\mathrm{h}} & =\frac{105}{8} \frac{M_{2} V}{b^{5}} \cos (3 \bar{\omega} \bar{t}), \\
\overline{\mathcal{B}}_{3 s}^{\mathrm{h}} & =\frac{105}{8} \frac{M_{2} V}{b^{5}} \sin (3 \bar{\omega} \bar{t}) .
\end{aligned}
$$

The harmonic components of the gravitoelectric moments can be substituted within the tidal potentials introduced in Sec. III With $\bar{\Omega}^{a}=[\sin \bar{\theta} \cos \bar{\phi}, \sin \bar{\theta} \sin \bar{\phi}, \cos \bar{\theta}]$, we have that

$$
\begin{aligned}
\overline{\mathcal{E}}^{\mathrm{a}}:= & \overline{\mathcal{E}}_{a b} \bar{\Omega}^{a} \bar{\Omega}^{b}=\frac{1}{2} \frac{M_{2}}{b^{3}}\left[1+\frac{1}{2} q_{1} V^{2}+2 \mathrm{PN}\right]\left(3 \cos ^{2} \bar{\theta}-1\right)-\frac{3}{2} \frac{M_{2}}{b^{3}}\left[1+\frac{1}{2}\left(q_{1}-4\right) V^{2}+2 \mathrm{PN}\right] \sin ^{2} \bar{\theta} \cos (2 \bar{\psi}) \\
\overline{\mathcal{E}}^{\circ}:= & \overline{\mathcal{E}}_{a b c} \bar{\Omega}^{a} \bar{\Omega}^{b} \bar{\Omega}^{c}=\frac{9}{4} \frac{M_{2}}{b^{4}}\left[1+\frac{1}{3}\left(9 q_{1}-8\right) V^{2}+2 \mathrm{PN}\right] \sin \bar{\theta}\left(5 \cos ^{2} \bar{\theta}-1\right) \cos (\bar{\psi}) \\
& -\frac{15}{4} \frac{M_{2}}{b^{4}}\left[1+\left(3 q_{1}-4\right) V^{2}+2 \mathrm{PN}\right] \sin ^{3} \bar{\theta} \cos (3 \bar{\psi}) \\
\overline{\mathcal{E}}^{\mathrm{h}}:= & \overline{\mathcal{E}}_{a b c d} \bar{\Omega}^{a} \bar{\Omega}^{b} \bar{\Omega}^{c} \bar{\Omega}^{d}=-\frac{9}{16} \frac{M_{2}}{b^{5}}\left[1+\frac{1}{14}\left(75 q_{1}-68\right) V^{2}+2 \mathrm{PN}\right]\left(35 \cos ^{4} \bar{\theta}-30 \cos ^{2} \bar{\theta}+3\right) \\
& +\frac{15}{4} \frac{M_{2}}{b^{5}}\left[1+\frac{3}{14}\left(25 q_{1}-24\right) V^{2}+2 \mathrm{PN}\right] \sin ^{2} \bar{\theta}\left(7 \cos ^{2} \bar{\theta}-1\right) \cos (2 \bar{\psi}), \\
& -\frac{105}{16} \frac{M_{2}}{b^{5}}\left[1+\frac{3}{14}\left(25 q_{1}-28\right) V^{2}+2 \mathrm{PN}\right] \sin ^{4} \bar{\theta} \cos (4 \bar{\psi}),
\end{aligned}
$$

where $\bar{\psi}:=\bar{\phi}-\bar{\omega} \bar{t}$.

\section{GEOMETRY OF A TIDALLY DEFORMED HORIZON}

The induced metric on the event horizon of a tidally deformed black hole was constructed in Sec. IV B of Ref. [30] - refer to their Eq. (4.3). This metric is expressed in terms of tidal moments defined in a "horizon calibration" that differs from the "post-Newtonian calibration" adopted in this work. The transformation between these calibrations was detailed in Sec. VI, and Eqs. (6.3) reveal that the tidal moments are identical up to corrections of order $v^{3}$ and beyond,

$$
\mathcal{E}_{a b}(\mathrm{H})=\mathcal{E}_{a b}(\mathrm{PN})+1.5 \mathrm{PN}, \quad \mathcal{E}_{a b c}(\mathrm{H})=\mathcal{E}_{a b c}(\mathrm{PN})+1.5 \mathrm{PN}
$$

and

$$
\mathcal{B}_{a b}(\mathrm{H})=\mathcal{B}_{a b}(\mathrm{PN})+1.5 \mathrm{PN}, \quad \mathcal{B}_{a b c}(\mathrm{H})=\mathcal{B}_{a b c}(\mathrm{PN})+1.5 \mathrm{PN}
$$

These corrections are of no concern in the determination of the horizon's geometry through 1PN order. We recall that $\mathcal{E}_{a b c d}$ and $\mathcal{B}_{a b c d}$ have a fixed calibration, and are therefore the same in the horizon and post-Newtonian calibrations. 
We begin with an examination of the quadrupole, octupole, and hexadecapole contributions to the induced metric, neglecting all contributions that are bilinear in the tidal moments. We have

$$
\gamma_{A B}=\left(2 M_{1}\right)^{2}\left[\Omega_{A B}-\frac{2}{3} M_{1}^{2}\left(\mathcal{E}_{A B}^{\mathrm{q}}+\mathcal{B}_{A B}^{\mathrm{q}}\right)-\frac{2}{15} M_{1}^{3}\left(\mathcal{E}_{A B}^{\circ}+\mathcal{B}_{A B}^{\circ}\right)-\frac{2}{105} M_{1}^{4}\left(\mathcal{E}_{A B}^{\mathrm{h}}+\mathcal{B}_{A B}^{\mathrm{h}}\right)\right],
$$

where the tidal potentials are given in the horizon calibration. This metric reflects a choice of coordinates on the horizon: $\theta^{A}$ is defined to be constant on the horizon's null generators, and the tidal moments are given as functions of the advanced-time coordinate $v$. To keep the notation uncluttered, we no longer make use of the overbar on the coordinates and tidal moments; it is now understood that all expressions refer to the black-hole frame.

Continuing to neglect all terms that are bilinear in the tidal moments, the Ricci scalar associated with the induced metric is given by

$$
\mathcal{R}=\frac{1}{2 M_{1}^{2}}\left(1-4 M_{1}^{2} \mathcal{E}^{\mathrm{q}}-\frac{4}{3} M_{1}^{3} \mathcal{E}^{\circ}-\frac{2}{7} M_{1}^{4} \mathcal{E}^{\mathrm{h}}\right),
$$

where $\mathcal{E}^{\mathrm{q}}, \mathcal{E}^{\circ}$, and $\mathcal{E}^{\mathrm{h}}$ are the tidal potentials introduced in Sec. III and evaluated for circular motion in Sec. IX E. There is no gravitomagnetic scalar potential, and $\mathcal{B}_{a b}, \mathcal{B}_{a b c}$ and $\mathcal{B}_{a b c d}$ do not appear in the Ricci scalar.

It is convenient to relate the horizon's intrinsic geometry to that of a fictitious two-dimensional surface embedded in a three-dimensional flat space. The surface is described by

$$
r=2 M_{1}\left[1+\varepsilon\left(\theta^{A}\right)\right]
$$

where $\varepsilon$ is a displacement function that represents the tidal deformation. With

$$
\varepsilon=-M_{1}^{2} \mathcal{E}^{\mathrm{a}}-\frac{2}{15} M_{1}^{3} \mathcal{E}^{\circ}-\frac{1}{63} M_{1}^{4} \mathcal{E}^{\mathrm{h}}
$$

the embedded surface possesses the same intrinsic Ricci scalar as the horizon.

Looking separately at each multipole component of the displacement function, we substitute Eqs. (9.36) and obtain

$$
\begin{aligned}
\varepsilon[\ell=2]= & -\frac{M_{1}^{2} M_{2}}{2 b^{3}}\left\{\left[1+\frac{1}{2} q_{1} V^{2}+2 \mathrm{PN}\right]\left(3 \cos ^{2} \theta-1\right)\right. \\
& \left.-3\left[1+\frac{1}{2}\left(q_{1}-4\right) V^{2}+2 \mathrm{PN}\right] \sin ^{2} \theta \cos (2 \psi)+1.5 \mathrm{PN}\right\} \\
\varepsilon[\ell=3]= & -\frac{M_{1}^{3} M_{2}}{10 b^{4}}\left\{3\left[1+\frac{1}{3}\left(9 q_{1}-8\right) V^{2}+2 \mathrm{PN}\right] \sin \theta\left(5 \cos ^{2} \theta-1\right) \cos (\psi)\right. \\
& \left.-5\left[1+\left(3 q_{1}-4\right) V^{2}+2 \mathrm{PN}\right] \sin ^{3} \theta \cos (3 \psi)+1.5 \mathrm{PN}\right\} \\
\varepsilon[\ell=4]= & -\frac{M_{1}^{4} M_{2}}{336 b^{5}}\left\{-3\left[1+\frac{1}{14}\left(75 q_{1}-68\right) V^{2}+2 \mathrm{PN}\right]\left(35 \cos ^{4} \theta-30 \cos ^{2} \theta+3\right)\right. \\
& +20\left[1+\frac{3}{14}\left(25 q_{1}-24\right) V^{2}+2 \mathrm{PN}\right] \sin ^{2} \theta\left(7 \cos ^{2} \theta-1\right) \cos (2 \psi), \\
& \left.-35\left[1+\frac{3}{14}\left(25 q_{1}-28\right) V^{2}+2 \mathrm{PN}\right] \sin ^{4} \theta \cos (4 \psi)+2 \mathrm{PN}\right\},
\end{aligned}
$$

where $\psi:=\phi-\bar{\omega} v$. The quadrupole displacement scales as $M_{1}^{2} M_{2} / b^{3}$, which we take to be of Newtonian order, and it features a 1PN correction of fractional order $V^{2}$. The octupole displacement comes with an additional factor of order $M_{1} / b=q_{1} V^{2}$ and therefore represents a 1PN correction to the quadrupole deformation. The hexadecapole displacement, with its additional factor of order $\left(M_{1} / b\right)^{2}$, represents a 2PN correction to the quadrupole displacement. The neglected bilinear terms would scale as $M_{1}^{4} M_{2}^{2} / b^{6}$ and therefore represent a 3PN correction to the leading term.

If we truncate the displacement function to an overall accuracy of 1PN order, we obtain

$$
\begin{aligned}
\varepsilon= & \frac{M_{1}^{2} M_{2}}{2 b^{3}}\left\{-\left[1+\frac{1}{2} q_{1} V^{2}\right]\left(3 \cos ^{2} \theta-1\right)+3\left[1+\frac{1}{2}\left(q_{1}-4\right) V^{2}\right] \sin ^{2} \theta \cos (2 \psi)\right. \\
& \left.-\frac{3}{5} q_{1} V^{2} \sin \theta\left(5 \cos ^{2} \theta-1\right) \cos (\psi)+q_{1} V^{2} \sin ^{3} \theta \cos (3 \psi)+1.5 \mathrm{PN}\right\} .
\end{aligned}
$$


As was discussed further in Sec. I, the displacement function describes a tidal bulge aligned with $\phi=\bar{\omega} v$.

A consequence of the black hole's tidal interaction is the fact that its mass slowly increases. The equation that describes this tidal heating is derived in Sec. IV D of Ref. [30. According to their Eqs. (4.14) and (4.16), the rate of change of the mass is given by

$$
\dot{M}_{1}=\dot{M}_{1}[\ell=2]+\dot{M}_{1}[\ell=3]+\text { higher order }
$$

where

$$
\begin{aligned}
& \dot{M}_{1}[\ell=2]=\frac{16}{45} M_{1}^{6}\left(\dot{\mathcal{E}}_{a b} \dot{\mathcal{E}}^{a b}+\dot{\mathcal{B}}_{a b} \dot{\mathcal{B}}^{a b}\right) \\
& \dot{M}_{1}[\ell=3]=\frac{16}{4725} M_{1}^{8}\left(\dot{\mathcal{E}}_{a b c} \dot{\mathcal{E}}^{a b c}+\frac{16}{9} \dot{\mathcal{B}}_{a b c} \dot{\mathcal{B}}^{a b c}\right)
\end{aligned}
$$

The influence of the hexadecapole moments on the mass is not currently known, and we therefore exclude it from the expression. We insert our previous expressions for the quadrupole and octupole contributions, and obtain

$$
\begin{aligned}
& \dot{M}_{1}[\ell=2]=\frac{32}{5} q_{1}^{6} q_{2}^{2}\left(\frac{M}{b}\right)^{9}\left[1+\left(q_{1}^{2}-6\right) V^{2}+1.5 \mathrm{PN}\right], \\
& \dot{M}_{1}[\ell=3]=\frac{64}{35} q_{1}^{8} q_{2}^{2}\left(\frac{M}{b}\right)^{11}\left[1+\left(q_{1}^{2}+5 q_{1}-\frac{175}{18}\right) V^{2}+1.5 \mathrm{PN}\right] .
\end{aligned}
$$

The leading, quadrupole term scales as $(M / b)^{9}=V^{18}$, and it represents a correction of 4PN order to the energy lost to gravitational waves. The octupole term scales as $(M / b)^{11}=V^{22}$; it represents a 4 PN correction to the quadrupole contribution, and a $8 \mathrm{PN}$ correction to the radiated energy.

\section{ACKNOWLEDGMENTS}

This work was supported by the Natural Sciences and Engineering Research Council of Canada.

\section{Appendix A: Decomposition of Cartesian tensors into irreducible pieces}

For our purposes in this Appendix, the irreducible pieces of a Cartesian tensor consist of its traces, symmetrictracefree (STF) pieces, antisymmetric pieces, and all mixtures among these basic building blocks. We need decompositions for fully symmetric tensors $S_{a b c \cdots}$ and for tensors $A_{j a b c \ldots}$ that are fully symmetric with respect to the $a b c \cdots$ indices. We use round brackets $(a b c \cdots)$ to denote the complete symmetrization of indices, square brackets $[a b c \cdots]$ to denote a complete antisymmetrization, and angular brackets $\langle a b c \cdots\rangle$ to denote an STF combination of indices (complete symmetrization and removal of all traces).

The decomposition of a fully symmetric tensor into irreducible pieces is accomplished with

$$
\begin{aligned}
{ }_{2} S_{a b}= & { }_{2} S_{\langle a b\rangle}+\frac{1}{3} \delta_{a b 2} S \\
{ }_{3} S_{a b c}= & { }_{3} S_{\langle a b c\rangle}+\frac{1}{5}\left(\delta_{a b 3} S_{c}+\delta_{a c 3} S_{b}+\delta_{b c 3} S_{a}\right) \\
{ }_{4} S_{a b c d}= & { }_{4} S_{\langle a b c d\rangle}+\frac{1}{7}\left(\delta_{a b 4} S_{\langle c d\rangle}+\delta_{a c 4} S_{\langle b d\rangle}+\delta_{a d} S_{\langle b c\rangle}+\delta_{b c 4} S_{\langle a d\rangle}+\delta_{b d 4} S_{\langle a c\rangle}+\delta_{c d 4} S_{\langle a b\rangle}\right) \\
& +\frac{1}{15}\left(\delta_{a b} \delta_{c d}+\delta_{a c} \delta_{b d}+\delta_{a d} \delta_{b c}\right)_{4} S \\
{ }_{5} S_{a b c d e}= & { }_{5} S_{\langle a b c d e\rangle}+\frac{1}{9}\left(\delta_{a b 5} S_{\langle c d e\rangle}+\delta_{a c} S_{\langle b d e\rangle}+\delta_{a d} S_{\langle b c e\rangle}+\delta_{a e} S_{\langle b c d\rangle}\right. \\
& \left.+\delta_{b c 5} S_{\langle a d e\rangle}+\delta_{b d}{ }_{5} S_{\langle a c e\rangle}+\delta_{b e}{ }_{5} S_{\langle a c d\rangle}+\delta_{c d}{ }_{5} S_{\langle a b e\rangle}+\delta_{c e}{ }_{5} S_{\langle a b d\rangle}+\delta_{d e} S_{\langle a b c\rangle}\right) \\
& +\frac{1}{35}\left[\left(\delta_{a b} \delta_{c d}+\delta_{a c} \delta_{b d}+\delta_{a d} \delta_{b c}\right)_{5} S_{e}+\left(\delta_{e a} \delta_{b c}+\delta_{e b} \delta_{a c}+\delta_{e c} \delta_{a b}\right){ }_{5} S_{d}+\left(\delta_{d e} \delta_{a b}+\delta_{d a} \delta_{e b}+\delta_{d b} \delta_{e a}\right)_{5} S_{c}\right. \\
& \left.+\left(\delta_{c d} \delta_{e a}+\delta_{c e} \delta_{d a}+\delta_{c a} \delta_{d e}\right)_{5} S_{b}+\left(\delta_{b c} \delta_{d e}+\delta_{b d} \delta_{c e}+\delta_{b e} \delta_{c d}\right)_{5} S_{a}\right]
\end{aligned}
$$


where ${ }_{2} S:={ }_{2} S_{a}{ }^{a},{ }_{3} S_{a}:={ }_{3} S_{a b}{ }^{b},{ }_{4} S_{\langle a b\rangle}$ is the STF piece of ${ }_{4} S_{a b c}{ }^{c},{ }_{4} S:={ }_{4} S_{a b}{ }_{a b},{ }_{5} S_{\langle a b c\rangle}$ is the STF piece of ${ }_{5} S_{a b c d}$, and ${ }_{5} S_{a}:={ }_{5} S_{a b c}{ }^{b c}$. The number before each tensor symbol indicates the rank of the parent tensor. The 6 independent components of ${ }_{2} S_{a b}$ are packaged into the 5 independent components of ${ }_{2} S_{\langle a b\rangle}$ and the single component of ${ }_{2} S$. The 10 independent components of ${ }_{3} S_{a b c}$ are packaged into the 7 independent components of ${ }_{3} S_{\langle a b c\rangle}$ and the 3 components of ${ }_{3} S_{a}$. The 15 independent components of ${ }_{4} S_{a b c d}$ are packaged into the 9 independent components of ${ }_{4} S_{\langle a b c d\rangle}$, the 5 independent components of ${ }_{4} S_{\langle a b\rangle}$, and the single component of ${ }_{4} S$. And finally, the 21 independent components of ${ }_{5} S_{a b c d e}$ are packaged into the 11 independent components of ${ }_{5} S_{\langle a b c d e\rangle}$, the 7 independent components of ${ }_{4} S_{\langle a b c\rangle}$, and the 3 components of ${ }_{5} S_{a}$.

The decomposition of a tensor $A_{j a b}=A_{j(a b)}$ is accomplished with

$$
{ }_{3} A_{j a b}={ }_{3} A_{\langle j a b\rangle}+\epsilon_{j a}{ }^{p}{ }_{3} A_{\langle p b\rangle}+\epsilon_{j b}{ }^{p}{ }_{3} A_{\langle p a\rangle}+\delta_{j a}\left({ }_{3} \bar{A}_{b}-{ }_{3} A_{b}\right)+\delta_{j b}\left({ }_{3} \bar{A}_{a}-{ }_{3} A_{a}\right)+\delta_{a b}\left({ }_{3} \bar{A}_{j}+2{ }_{3} A_{j}\right),
$$

where

$$
\begin{aligned}
{ }_{3} \bar{A}_{a} & :=\frac{1}{15}\left({ }_{3} A_{a p}{ }^{p}+2{ }_{3} A_{p a}^{p}\right), \\
{ }_{3} A_{a} & :=\frac{1}{6}\left({ }_{3} A_{a p}{ }^{p}-{ }_{3} A^{p}{ }_{p a}\right), \\
{ }_{3} A_{\langle a b\rangle} & :=\frac{1}{3} \epsilon_{p q(a 33} A_{b)}^{p q} .
\end{aligned}
$$

The 18 independent components of ${ }_{3} A_{j a b}$ are packaged into the 7 independent components of ${ }_{3} A_{\langle j a b\rangle}$, the 5 independent components of ${ }_{3} A_{\langle a b\rangle}$, the 3 components of ${ }_{3} A_{a}$, and the 3 components of ${ }_{3} \bar{A}_{a}$.

The decomposition of a tensor $A_{j a b c}=A_{j(a b c)}$ into irreducible pieces is given by

$$
\begin{aligned}
{ }_{4} A_{j a b c}= & { }_{4} A_{\langle j a b c\rangle}+\epsilon_{j a}{ }^{p}{ }_{4} A_{\langle p b c\rangle}+\epsilon_{j c}{ }^{p}{ }_{4} A_{\langle p a b\rangle}+\epsilon_{j b}{ }^{p}{ }_{4} A_{\langle p c a\rangle} \\
& +\delta_{j a}\left({ }_{4} \bar{A}_{\langle b c\rangle}+{ }_{4} A_{\langle b c\rangle}\right)+\delta_{j c}\left({ }_{4} \bar{A}_{\langle a b\rangle}+{ }_{4} A_{\langle a b\rangle}\right)+\delta_{j b}\left({ }_{4} \bar{A}_{\langle c a\rangle}+{ }_{4} A_{\langle c a\rangle}\right) \\
& +\delta_{a b}\left({ }_{4} \bar{A}_{\langle j c\rangle}-{ }_{4} A_{\langle j c\rangle}\right)+\delta_{a c}\left({ }_{4} \bar{A}_{\langle j b\rangle}-{ }_{4} A_{\langle j b\rangle}\right)+\delta_{b c}\left({ }_{4} \bar{A}_{\langle j a\rangle}-{ }_{4} A_{\langle j a\rangle}\right) \\
& +\left(\delta_{a b} \epsilon_{j c}{ }^{p}+\delta_{a c} \epsilon_{j b}{ }^{p}+\delta_{b c} \epsilon_{j a}{ }^{p}\right){ }_{4} A_{p}+\left(\delta_{j a} \delta_{b c}+\delta_{j b} \delta_{a c}+\delta_{j c} \delta_{a b}\right){ }_{4} \bar{A},
\end{aligned}
$$

where

$$
\begin{aligned}
{ }_{4} \bar{A} & :=\frac{1}{15}{ }_{4} A^{p}{ }_{p q}^{q}, \\
{ }_{4} \bar{A}_{\langle a b\rangle} & :=\frac{1}{28}\left({ }_{4} A_{a b p}{ }^{p}+A_{b a p}{ }^{p}+2{ }_{4} A_{p a b}^{p}\right)-\frac{1}{21} \delta_{a b 4} A^{p}{ }_{p q}^{q} \\
{ }_{4} A_{a} & :=\frac{1}{10} \epsilon_{a p q 4} A_{r}^{p q r}, \\
{ }_{4} A_{\langle a b\rangle} & :=\frac{1}{12}\left(2{ }_{4} A_{p a b}^{p}-{ }_{4} A_{a b p}{ }^{p}-{ }_{4} A_{b a p}{ }^{p}\right), \\
{ }_{4} A_{\langle a b c\rangle} & :=\frac{1}{4} \epsilon_{p q\left(a{ }_{4}\right.} A^{p q}{ }_{b c)}-\frac{1}{6}\left(\delta_{a b} A_{4}+\delta_{a c 4} A_{b}+\delta_{b c}{ }_{4} A_{a}\right) .
\end{aligned}
$$

The 30 independent components of ${ }_{4} A_{j a b c}$ are packaged into the 9 independent components of ${ }_{4} A_{\langle j a b c\rangle}$, the 7 independent components of ${ }_{4} A_{\langle a b c\rangle}$, the 5 independent components of ${ }_{4} A_{\langle a b\rangle}$, the 5 independent components of ${ }_{4} \bar{A}_{\langle a b\rangle}$, the 3 components of ${ }_{4} A_{a}$, and the single component of ${ }_{4} \bar{A}$.

The decomposition of a tensor $A_{j a b c d}=A_{j(a b c d)}$ is accomplished with

$$
\begin{aligned}
{ }_{5} A_{j a b c d}:= & { }_{5} A_{\langle j a b c d\rangle}+\epsilon_{j a}{ }_{5}{ }_{5} A_{\langle p b c d\rangle}+\epsilon_{j d}{ }_{5} A_{\langle p a b c\rangle}+\epsilon_{j c}{ }^{p}{ }_{5} A_{\langle p d a b\rangle}+\epsilon_{j b}{ }_{5} A_{\langle p c d a\rangle} \\
& +\delta_{j a}\left({ }_{5} \bar{A}_{\langle b c d\rangle}-3{ }_{5} A_{\langle b c d\rangle}\right)+\delta_{j b}\left({ }_{5} \bar{A}_{\langle c d a\rangle}-3{ }_{5} A_{\langle c d a\rangle}\right)+\delta_{j c}\left({ }_{5} \bar{A}_{\langle d a b\rangle}-3_{5} A_{\langle d a b\rangle}\right)+\delta_{j d}\left({ }_{5} \bar{A}_{\langle a b c\rangle}-3{ }_{5} A_{\langle a b c\rangle}\right) \\
& +\delta_{a b}\left({ }_{5} \bar{A}_{\langle j c d\rangle}+2{ }_{5} A_{\langle j c d\rangle}\right)+\delta_{a c}\left({ }_{5} \bar{A}_{\langle j b d\rangle}+2{ }_{5} A_{\langle j b d\rangle}\right)+\delta_{a d}\left({ }_{5} \bar{A}_{\langle j b c\rangle}+2_{5} A_{\langle j b c\rangle}\right)+\delta_{b c}\left({ }_{5} \bar{A}_{\langle j a d\rangle}+2{ }_{5} A_{\langle j a d\rangle}\right) \\
& +\delta_{b d}\left({ }_{5} \bar{A}_{\langle j a c\rangle}+2{ }_{5} A_{\langle j a c\rangle}\right)+\delta_{c d}\left({ }_{5} \bar{A}_{\langle j a b\rangle}+2{ }_{5} A_{\langle j a b\rangle}\right) \\
& +\epsilon_{j a}{ }^{p}\left(\delta_{b c 5} A_{\langle p d\rangle}+\delta_{d b 5} A_{\langle p c\rangle}+\delta_{c d} A_{\langle p b\rangle}\right)+\epsilon_{j d}{ }^{p}\left(\delta_{a b 5} A_{\langle p c\rangle}+\delta_{c a} A_{\langle p b\rangle}+\delta_{b c 5} A_{\langle p a\rangle}\right) \\
& +\epsilon_{j c}{ }^{p}\left(\delta_{d a}{ }_{5} A_{\langle p b\rangle}+\delta_{b d}{ }_{5} A_{\langle p a\rangle}+\delta_{a b 5} A_{\langle p d\rangle}\right)+\epsilon_{j b}{ }^{p}\left(\delta_{c d 5} A_{\langle p a\rangle}+\delta_{a c 5} A_{\langle p d\rangle}+\delta_{d a}{ }_{5} A_{\langle p c\rangle}\right) \\
& +\left(\delta_{j a} \delta_{b c}+\delta_{j c} \delta_{a b}+\delta_{j b} \delta_{c a}\right)\left({ }_{5} \bar{A}_{d}+{ }_{5} A_{d}\right)+\left(\delta_{j d} \delta_{a b}+\delta_{j b} \delta_{d a}+\delta_{j a} \delta_{b d}\right)\left({ }_{5} \bar{A}_{c}+{ }_{5} A_{c}\right) \\
& +\left(\delta_{j c} \delta_{d a}+\delta_{j a} \delta_{c d}+\delta_{j d} \delta_{a c}\right)\left({ }_{5} \bar{A}_{b}+{ }_{5} A_{b}\right)+\left(\delta_{j b} \delta_{c d}+\delta_{j d} \delta_{b c}+\delta_{j c} \delta_{d b}\right)\left({ }_{5} \bar{A}_{a}+{ }_{5} A_{a}\right)
\end{aligned}
$$




$$
+\left(\delta_{a b} \delta_{c d}+\delta_{a c} \delta_{b d}+\delta_{a d} \delta_{b c}\right)\left({ }_{5} \bar{A}_{j}-4_{5} A_{j}\right)
$$

where

$$
\begin{aligned}
& { }_{5} \bar{A}_{a}:=\frac{1}{175}\left({ }_{5} A_{a p q}^{p q}+4{ }_{5} A_{p a q}^{p}\right), \\
& { }_{5} \bar{A}_{\langle a b c\rangle}:=\frac{1}{45}\left({ }_{5} A_{a b c p}^{p}+{ }_{5} A_{c a b p}{ }^{p}+{ }_{5} A_{b c a p}{ }^{p}+2{ }_{5} A^{p}{ }_{p a b c}\right)-\frac{7}{9}\left(\delta_{a b 5} \bar{A}_{c}+\delta_{a c 5} \bar{A}_{b}+\delta_{b c 5} \bar{A}_{a}\right), \\
& { }_{5} A_{a}:=\frac{1}{50}\left({ }_{5} A^{p}{ }_{p a q}^{q}-{ }_{5} A_{a p q}{ }^{p q}\right), \\
& { }_{5} A_{\langle a b\rangle}:=\frac{1}{21} \epsilon_{p q(a 5} A_{b) r}^{p q} \\
& { }_{5} A_{\langle a b c\rangle}:=\frac{1}{60}\left({ }_{5} A_{a b c p}^{p}+{ }_{5} A_{c a b p}{ }^{p}+{ }_{5} A_{b c a p}{ }^{p}-3{ }_{5} A^{p}{ }_{p a b c}\right)+\frac{1}{6}\left(\delta_{a b 5} A_{c}+\delta_{a c 5} A_{b}+\delta_{b c 5} A_{a}\right), \\
& { }_{5} A_{\langle a b c d\rangle}:=\frac{1}{5} \epsilon_{p q\left(a{ }_{5}\right.} A_{b c d)}^{p q}-\frac{3}{10}\left(\delta_{a b 5} A_{\langle c d\rangle}+\delta_{a c 5} A_{\langle b d\rangle}+\delta_{a d 5} A_{\langle b c\rangle}+\delta_{b c} A_{\langle a d\rangle}+\delta_{b d 5} A_{\langle a c\rangle}+\delta_{c d 5} A_{\langle a b\rangle}\right) \text {. }
\end{aligned}
$$

The 45 independent components of ${ }_{5} A_{j a b c d}$ are packaged into the 11 independent components of ${ }_{5} A_{\langle j a b c d\rangle}$, the 9 independent components of ${ }_{5} A_{\langle a b c d\rangle}$, the 7 independent components of ${ }_{5} A_{\langle a b c\rangle}$, the 7 independent components of ${ }_{5} \bar{A}_{\langle a b c\rangle}$, the 5 independent components of ${ }_{5} A_{\langle a b\rangle}$, the 3 components of ${ }_{5} A_{a}$, and the 3 components of ${ }_{5} \bar{A}_{a}$.

The decompositions of Eqs. (A1) are familiar (see, for example, Sec. 1.5.3 of Ref. [34]), and there is no need to provide a derivation. The decompositions of Eqs. A2, (A4), and (A6) are perhaps less familiar, and we snow sketch their derivation.

Equation A2 can be obtained from the identity $A_{j a b}=A_{(j a b)}+\frac{2}{3}\left(A_{[j a] b}+A_{[j b] a}\right)$. The first term is decomposed into trace and STF pieces, and the second and third terms are re-expressed in terms of a general tensor $X_{p a}$ according to $A_{[j a] b}=\epsilon_{j a}{ }^{p} X_{p b}$. In the next step, $X_{a b}$ is decomposed into trace, STF, and antisymmetric pieces according to $X_{a b}=\frac{1}{3} \delta_{a b} X+X_{\langle a b\rangle}+\epsilon_{a b}{ }^{p} Y_{p}$, where $X:=X_{p}{ }^{p}$ and $Y_{p}$ is a vector. Making the substitutions and simplifying returns Eq. A2 after introducing the definitions of Eqs. A3, with ${ }_{3} \bar{A}_{a}$ representing the trace part of $A_{(j a b)},{ }_{3} A_{a}$ substituting for $Y_{a}$, and ${ }_{3} A_{\langle a b\rangle}$ substituting for $X_{\langle a b\rangle} ; X$ does not appear in the final expression.

Equation $\mathrm{A} 4$ is obtained in a similar way. The starting identity is $A_{j a b c}=A_{(j a b c)}+\frac{1}{2}\left(A_{[j a] b c}+A_{[j c] a b}+A_{[j b] c a}\right)$, and the first term is decomposed into trace and STF pieces, while the remaining terms are rewritten in terms of a tensor $X_{p a b}$ according to $A_{[j a] b c}=\epsilon_{j a}{ }^{p} X_{p b c}$. Because $X_{p a b}$ is symmetric in the last two indices, it can be decomposed according to Eq. A2, Making the substitutions, simplifying, and introducing the definitions of Eqs. A5 returns Eq. A4).

For Eq. A6 we begin with $A_{j a b c d}=A_{(j a b c d)}+\frac{2}{5}\left(A_{[j a] b c d}+A_{[j d] a b c}+A_{[j c] d a b}+A_{[j b] c d a}\right)$ and write $A_{[j a] b c d}=\epsilon_{j a}{ }^{p} X_{p b c d}$, with $X_{p b c d}$ symmetric in the last three indices. This tensor can be decomposed according to Eq. A4 and Eq. A6 eventually follows.

\section{Appendix B: Derivatives of distance function}

We introduce the Cartesian vector $s:=\boldsymbol{x}-\boldsymbol{r}(t)$, in which $\boldsymbol{x}$ denotes the variable position of a point in threedimensional flat space, and $\boldsymbol{r}(t)$ is a known vectorial function of time. We let $\boldsymbol{v}:=d \boldsymbol{r} / d t$ and $\boldsymbol{a}:=d \boldsymbol{v} / d t$. The "distance function" of the title is defined by $s:=|s|$, and we also introduce the unit vector $\hat{\boldsymbol{s}}:=\boldsymbol{s} / s$. We let $v_{s}:=\boldsymbol{v} \cdot \hat{\boldsymbol{s}}$ and $a_{s}:=\boldsymbol{a} \cdot \hat{\boldsymbol{s}}$. Below we record a number of derivatives of the distance function, which are needed in the main text. These results are easily derived from the elementary identities

$$
\begin{aligned}
\partial_{a} s & =\hat{s}_{a} \\
\partial_{a} \hat{s}_{b} & =\frac{1}{s}\left(\delta_{a b}-\hat{s}_{a} \hat{s}_{b}\right), \\
\partial_{t} s & =-v_{s}, \\
\partial_{t} \hat{s}_{a} & =-\frac{1}{s}\left(v_{a}-v_{s} \hat{s}_{a}\right), \\
\partial_{a} v_{s} & =\frac{1}{s}\left(v_{a}-v_{s} \hat{s}_{a}\right), \\
\partial_{a} a_{s} & =\frac{1}{s}\left(a_{a}-a_{s} \hat{s}_{a}\right),
\end{aligned}
$$




$$
\partial_{t} v_{s}=a_{s}-\frac{1}{s}\left(v^{2}-v_{s}^{2}\right),
$$

where $v^{2}:=\boldsymbol{v} \cdot \boldsymbol{v}$.

Derivatives of $s^{-1}$ are given by

$$
\begin{aligned}
\partial_{a} s^{-1}= & -\frac{1}{s^{2}} \hat{s}_{a} \\
\partial_{a b} s^{-1}= & \frac{3}{s^{3}} \hat{s}_{\langle a b\rangle}=\frac{3}{s^{3}}\left(\hat{s}_{a} \hat{s}_{b}-\frac{1}{3} \delta_{a b}\right) \\
\partial_{a b c} s^{-1}= & -\frac{15}{s^{4}} \hat{s}_{\langle a b c\rangle}=-\frac{15}{s^{4}}\left[\hat{s}_{a} \hat{s}_{b} \hat{s}_{c}-\frac{1}{5}\left(\delta_{a b} \hat{s}_{c}+\delta_{a c} \hat{s}_{b}+\delta_{b c} \hat{s}_{a}\right)\right] \\
\partial_{a b c d} s^{-1}= & \frac{105}{s^{5}} \hat{s}_{\langle a b c d\rangle}=\frac{105}{s^{5}}\left[\hat{s}_{a} \hat{s}_{b} \hat{s}_{c} \hat{s}_{d}-\frac{1}{7}\left(\delta_{a b} \hat{s}_{c} \hat{s}_{d}+\delta_{a c} \hat{s}_{b} \hat{s}_{d}+\delta_{a d} \hat{s}_{b} \hat{s}_{c}+\delta_{b c} \hat{s}_{a} \hat{s}_{d}+\delta_{b d} \hat{s}_{a} \hat{s}_{c}+\delta_{c d} \hat{s}_{a} \hat{s}_{b}\right)\right. \\
& \left.+\frac{1}{35}\left(\delta_{a b} \delta_{c d}+\delta_{a c} \delta_{b d}+\delta_{a d} \delta_{b c}\right)\right]
\end{aligned}
$$

and

$$
\begin{aligned}
\partial_{t a} s^{-1}= & \frac{1}{s^{3}}\left(-3 v_{s} \hat{s}_{a}+v_{a}\right) \\
\partial_{t a b} s^{-1}= & \frac{1}{s^{4}}\left[15 v_{s} \hat{s}_{a} \hat{s}_{b}-3\left(v_{a} \hat{s}_{b}+\hat{s}_{a} v_{b}\right)-3 v_{s} \delta_{a b}\right], \\
\partial_{t a b c} s^{-1}= & \frac{1}{s^{5}}\left[-105 v_{s} \hat{s}_{a} \hat{s}_{b} \hat{s}_{c}+15\left(v_{a} \hat{s}_{b} \hat{s}_{c}+\hat{s}_{a} v_{b} \hat{s}_{c}+\hat{s}_{a} \hat{s}_{b} v_{c}\right)+15 v_{s}\left(\delta_{a b} \hat{s}_{c}+\delta_{a c} \hat{s}_{b}+\delta_{b c} \hat{s}_{a}\right)\right. \\
& \left.-3\left(\delta_{a b} v_{c}+\delta_{a c} v_{b}+\delta_{b c} v_{a}\right)\right] .
\end{aligned}
$$

Derivatives of $s$ are given by

$$
\begin{aligned}
\partial_{a b} s= & \frac{1}{s}\left(-\hat{s}_{a} \hat{s}_{b}+\delta_{a b}\right) \\
\partial_{a b c} s= & \frac{1}{s^{2}}\left[3 \hat{s}_{a} \hat{s}_{b} \hat{s}_{c}-\left(\delta_{a b} \hat{s}_{c}+\delta_{a c} \hat{s}_{b}+\delta_{b c} \hat{s}_{a}\right)\right] \\
\partial_{a b c d} s= & \frac{1}{s^{3}}\left[-15 \hat{s}_{a} \hat{s}_{b} \hat{s}_{c} \hat{s}_{d}+3\left(\delta_{a b} \hat{s}_{c} \hat{s}_{d}+\delta_{a c} \hat{s}_{b} \hat{s}_{d}+\delta_{a d} \hat{s}_{b} \hat{s}_{c}+\delta_{b c} \hat{s}_{a} \hat{s}_{d}+\delta_{b d} \hat{s}_{a} \hat{s}_{c}+\delta_{c d} \hat{s}_{a} \hat{s}_{b}\right)\right. \\
& \left.-\left(\delta_{a b} \delta_{c d}+\delta_{a c} \delta_{b d}+\delta_{a d} \delta_{b c}\right)\right]
\end{aligned}
$$

and

$$
\begin{aligned}
\partial_{t a b} s= & \frac{1}{s^{3}}\left[-3 v_{s} \hat{s}_{a} \hat{s}_{b}+\left(v_{a} \hat{s}_{b}+\hat{s}_{a} v_{b}\right)+v_{s} \delta_{a b}\right] \\
\partial_{t a b c} s= & \frac{1}{s^{3}}\left[15 v_{s} \hat{s}_{a} \hat{s}_{b} \hat{s}_{c}-3\left(v_{a} \hat{s}_{b} \hat{s}_{c}+\hat{s}_{a} v_{b} \hat{s}_{c}+\hat{s}_{a} \hat{s}_{b} v_{c}\right)-3 v_{s}\left(\delta_{a b} \hat{s}_{c}+\delta_{a c} \hat{s}_{b}+\delta_{b c} \hat{s}_{a}\right)\right. \\
& \left.+\left(\delta_{a b} v_{c}+\delta_{a c} v_{b}+\delta_{b c} v_{a}\right)\right], \\
\partial_{t a b c d} s= & \frac{1}{s^{4}}\left[-105 v_{s} \hat{s}_{a} \hat{s}_{b} \hat{s}_{c} \hat{s}_{d}+15\left(v_{a} \hat{s}_{b} \hat{s}_{c} \hat{s}_{d}+\hat{s}_{a} v_{b} \hat{s}_{c} \hat{s}_{d}+\hat{s}_{a} \hat{s}_{b} v_{c} \hat{s}_{d}+\hat{s}_{a} \hat{s}_{b} \hat{s}_{c} v_{d}\right)\right. \\
& +15 v_{s}\left(\delta_{a b} \hat{s}_{c} \hat{s}_{d}+\delta_{a c} \hat{s}_{b} \hat{s}_{d}+\delta_{a d} \hat{s}_{b} \hat{s}_{c}+\delta_{b c} \hat{s}_{a} \hat{s}_{d}+\delta_{b d} \hat{s}_{a} \hat{s}_{c}+\delta_{c d} \hat{s}_{a} \hat{s}_{b}\right) \\
& -6\left(\delta_{a b} v_{(c} \hat{s}_{d)}+\delta_{a c} v_{(b} \hat{s}_{d)}+\delta_{a d} v_{(b} \hat{s}_{c)}+\delta_{b c} v_{(a} \hat{s}_{d)}+\delta_{b d} v_{(a} \hat{s}_{c)}+\delta_{c d} v_{(a} \hat{s}_{b)}\right) \\
& \left.-3 v_{s}\left(\delta_{a b} \delta_{c d}+\delta_{a c} \delta_{b d}+\delta_{a d} \delta_{b c}\right)\right]
\end{aligned}
$$

and

$$
\begin{aligned}
\partial_{t t a b} s= & \frac{1}{s^{2}}\left[-3 a_{s} \hat{s}_{a} \hat{s}_{b}+\left(a_{a} \hat{s}_{b}+\hat{s}_{a} a_{b}\right)+a_{s} \delta_{a b}\right] \\
& +\frac{1}{s^{3}}\left[3\left(v^{2}-5 v_{s}^{2}\right) \hat{s}_{a} \hat{s}_{b}+6 v_{s}\left(v_{a} \hat{s}_{b}+\hat{s}_{a} v_{b}\right)-2 v_{a} v_{b}-\left(v^{2}-3 v_{s}^{2}\right) \delta_{a b}\right],
\end{aligned}
$$




$$
\begin{aligned}
& \partial_{t t a b c} s=\frac{1}{s^{3}}\left[15 a_{s} \hat{s}_{a} \hat{s}_{b} \hat{s}_{c}-3\left(a_{a} \hat{s}_{b} \hat{s}_{c}+\hat{s}_{a} a_{b} \hat{s}_{c}+\hat{s}_{a} \hat{s}_{b} a_{c}\right)-3 a_{s}\left(\delta_{a b} \hat{s}_{c}+\delta_{a c} \hat{s}_{b}+\delta_{b c} \hat{s}_{a}\right)+\left(\delta_{a b} a_{c}+\delta_{a c} a_{b}+\delta_{b c} a_{a}\right)\right] \\
& +\frac{1}{s^{4}}\left[-15\left(v^{2}-7 v_{s}^{2}\right) \hat{s}_{a} \hat{s}_{b} \hat{s}_{c}-30 v_{s}\left(v_{a} \hat{s}_{b} \hat{s}_{c}+\hat{s}_{a} v_{b} \hat{s}_{c}+\hat{s}_{a} \hat{s}_{b} v_{c}\right)+3\left(v^{2}-5 v_{s}^{2}\right)\left(\delta_{a b} \hat{s}_{c}+\delta_{a c} \hat{s}_{b}+\delta_{b c} \hat{s}_{a}\right)\right. \\
& \left.+6\left(v_{a} v_{b} \hat{s}_{c}+v_{a} \hat{s}_{b} v_{c}+\hat{s}_{a} v_{b} v_{c}\right)+6 v_{s}\left(\delta_{a b} v_{c}+\delta_{a c} v_{b}+\delta_{b c} v_{a}\right)\right] \text {, } \\
& \partial_{t t a b c d} s=\frac{1}{s^{4}}\left[-105 a_{s} \hat{s}_{a} \hat{s}_{b} \hat{s}_{c} \hat{s}_{d}+15\left(a_{a} \hat{s}_{b} \hat{s}_{c} \hat{s}_{d}+\hat{s}_{a} a_{b} \hat{s}_{c} \hat{s}_{d}+\hat{s}_{a} \hat{s}_{b} a_{c} \hat{s}_{d}+\hat{s}_{a} \hat{s}_{b} \hat{s}_{c} a_{d}\right)\right. \\
& +15 a_{s}\left(\delta_{a b} \hat{s}_{c} \hat{s}_{d}+\delta_{a c} \hat{s}_{b} \hat{s}_{d}+\delta_{a d} \hat{s}_{b} \hat{s}_{c}+\delta_{b c} \hat{s}_{a} \hat{s}_{d}+\delta_{b d} \hat{s}_{a} \hat{s}_{c}+\delta_{c d} \hat{s}_{a} \hat{s}_{b}\right) \\
& \left.-12\left(\delta_{a b} a_{(c} \hat{s}_{d)}+\delta_{a c} a_{(b} \hat{s}_{d)}+\delta_{a d} a_{(b} \hat{s}_{c)}+\delta_{b c} a_{(a} \hat{s}_{d)}+\delta_{b d} a_{(a} \hat{s}_{c)}+\delta_{c d} a_{(a} \hat{s}_{b)}\right)-3 a_{s}\left(\delta_{a b} \delta_{c d}+\delta_{a c} \delta_{b d}+\delta_{a d} \delta_{b c}\right)\right] \\
& +\frac{1}{s^{5}}\left[105\left(v^{2}-9 v_{s}^{2}\right) \hat{s}_{a} \hat{s}_{b} \hat{s}_{c} \hat{s}_{d}+210 v_{s}\left(v_{a} \hat{s}_{b} \hat{s}_{c} \hat{s}_{d}+\hat{s}_{a} v_{b} \hat{s}_{c} \hat{s}_{d}+\hat{s}_{a} \hat{s}_{b} v_{c} \hat{s}_{d}+\hat{s}_{a} \hat{s}_{b} \hat{s}_{c} v_{d}\right)\right. \\
& -30\left(v_{a} v_{b} \hat{s}_{c} \hat{s}_{d}+v_{a} \hat{s}_{b} v_{c} \hat{s}_{d}+v_{a} \hat{s}_{b} \hat{s}_{c} v_{d}+\hat{s}_{a} v_{b} v_{c} \hat{s}_{d}+\hat{s}_{a} v_{b} \hat{s}_{c} v_{d}+\hat{s}_{a} \hat{s}_{b} v_{c} v_{d}\right) \\
& -15\left(v^{2}-7 v_{s}^{2}\right)\left(\delta_{a b} \hat{s}_{c} \hat{s}_{d}+\delta_{a c} \hat{s}_{b} \hat{s}_{d}+\delta_{a d} \hat{s}_{b} \hat{s}_{c}+\delta_{b c} \hat{s}_{a} \hat{s}_{d}+\delta_{b d} \hat{s}_{a} \hat{s}_{c}+\delta_{c d} \hat{s}_{a} \hat{s}_{b}\right) \\
& -60 v_{s}\left(\delta_{a b} v_{(c} \hat{s}_{d)}+\delta_{a c} v_{(b} \hat{s}_{d)}+\delta_{a d} v_{(b} \hat{s}_{c)}+\delta_{b c} v_{(a} \hat{s}_{d)}+\delta_{b d} v_{(a} \hat{s}_{c)}+\delta_{c d} v_{(a} \hat{s}_{b)}\right) \\
& +6\left(\delta_{a b} v_{c} v_{d}+\delta_{a c} v_{b} v_{d}+\delta_{a d} v_{b} v_{c}+\delta_{b c} v_{a} v_{d}+\delta_{b d} v_{a} v_{c}+\delta_{c d} v_{a} v_{b}\right) \\
& \left.+3\left(v^{2}-5 v_{s}^{2}\right)\left(\delta_{a b} \delta_{c d}+\delta_{a c} \delta_{b d}+\delta_{a d} \delta_{b c}\right)\right] \text {. }
\end{aligned}
$$

\section{Appendix C: Tables}

TABLE I. Irreducible tidal potentials. The superscripts q, o, and h stand for "quadrupole," "octupole," and "hexadecapole," respectively. The factor of 2 in $\mathcal{E}^{\mathrm{h}}$ is inserted to respect Zhang's normalization convention for $\mathcal{E}_{a b c d}$; see Eqs. (2.3). The factors of $\frac{4}{3}$ and $\frac{10}{3}$ in $\mathcal{B}_{a}^{\circ}$ and $\mathcal{B}_{a}^{\mathrm{h}}$ are inserted to respect Zhang's normalization convention for $\mathcal{B}_{a b c}$ and $\mathcal{B}_{a b c d}$; see Eqs. 2.2 , and 2.3 .

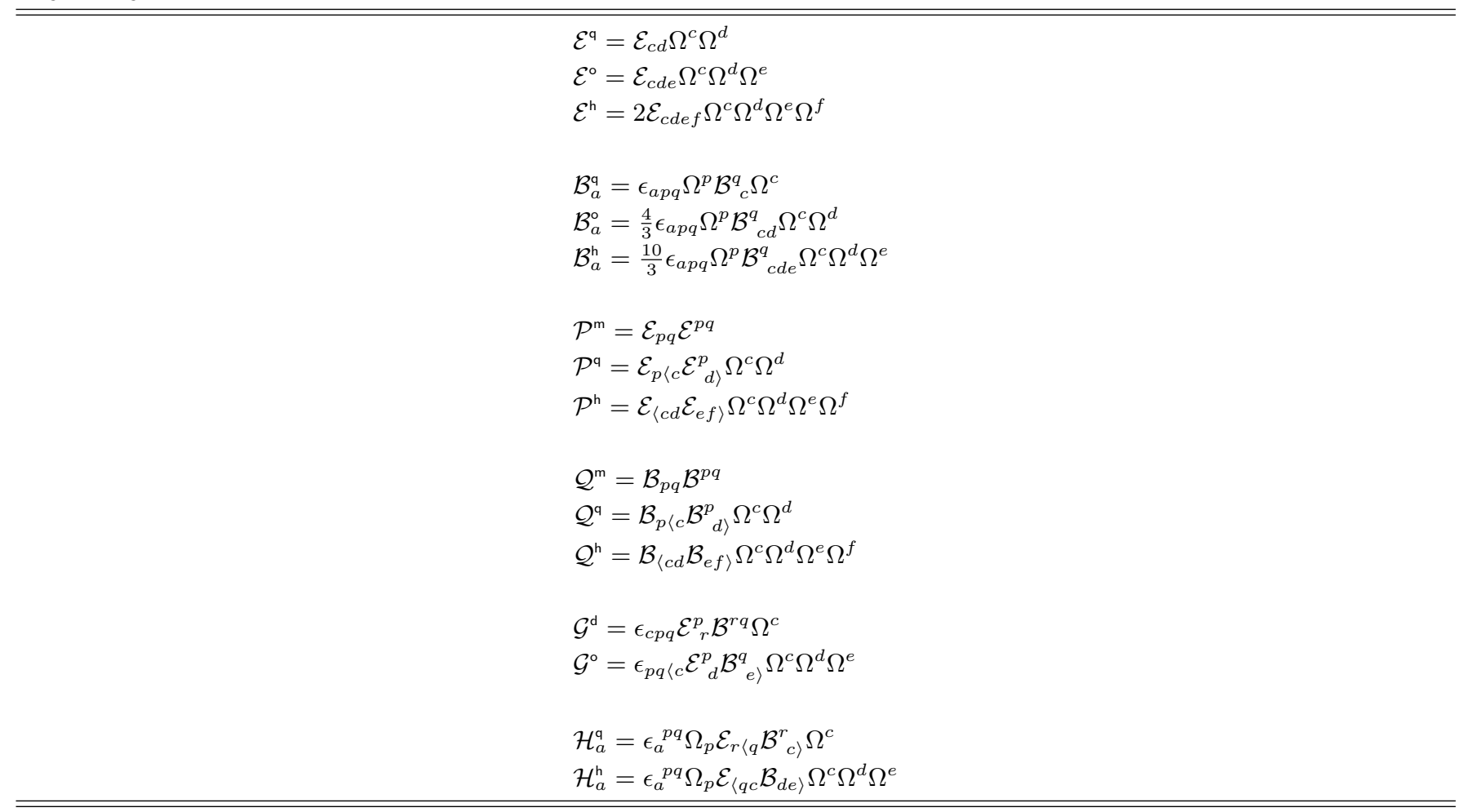


TABLE II. Spherical-harmonic functions $Y^{\ell m}$ and harmonic components $\mathcal{A}_{m}^{(\ell)}$ involved in the decomposition of $\mathcal{A}^{(\ell)}:=$ $\mathcal{A}_{\left\langle k_{1} \cdots k_{\ell}\right\rangle} \Omega^{k_{1}} \cdots \Omega^{k_{\ell}}=\sum_{m} \mathcal{A}_{m}^{(\ell)} Y^{\ell m}$. The functions are real, and they are listed for the relevant modes $\ell=1$ (dipole), $\ell=2$, (quadrupole), $\ell=3$ (octupole), and $\ell=4$ (hexadecapole). The abstract index $m$ describes the dependence of these functions on the angle $\phi$; for example $Y^{\ell, 2 s}$ is proportional to $\sin 2 \phi$. We write $C:=\cos \theta$ and $S:=\sin \theta$. The harmonic components are expressed in terms of the independent components of the STF tensor $\mathcal{A}_{\left\langle k_{1} \cdots k_{\ell}\right\rangle}$.

\begin{tabular}{|c|c|}
\hline$Y^{1,0}=C$ & $\mathcal{A}_{0}^{\mathrm{d}}=\mathcal{A}_{3}$ \\
\hline$Y^{1,1 c}=S \cos \phi$ & $\mathcal{A}_{1 c}^{\mathrm{d}}=\mathcal{A}_{1}$ \\
\hline$Y^{1,1 s}=S \sin \phi$ & $\mathcal{A}_{1 s}^{\mathrm{d}}=\mathcal{A}_{2}$ \\
\hline$Y^{2,0}=1-3 C^{2}$ & $\mathcal{A}_{0}^{\mathrm{q}}=\frac{1}{2}\left(\mathcal{A}_{11}+\mathcal{A}_{22}\right)$ \\
\hline$Y^{2,1 c}=2 S C \cos \phi$ & $\mathcal{A}_{1 c}^{\mathrm{q}}=\mathcal{A}_{13}$ \\
\hline$Y^{2,1 s}=2 S C \sin \phi$ & $\mathcal{A}_{1 s}^{\mathrm{q}}=\mathcal{A}_{23}$ \\
\hline$Y^{2,2 c}=S^{2} \cos 2 \phi$ & $\mathcal{A}_{2 c}^{\mathrm{q}}=\frac{1}{2}\left(\mathcal{A}_{11}-\mathcal{A}_{22}\right)$ \\
\hline$Y^{2,2 s}=S^{2} \sin 2 \phi$ & $\mathcal{A}_{2 s}^{\mathrm{q}}=\mathcal{A}_{12}$ \\
\hline$Y^{3,0}=C\left(3-5 C^{2}\right)$ & $\mathcal{A}_{0}^{\circ}=\frac{1}{2}\left(\mathcal{A}_{113}+\mathcal{A}_{223}\right)$ \\
\hline$Y^{3,1 c}=\frac{3}{2} S\left(1-5 C^{2}\right) \cos \phi$ & $\mathcal{A}_{1 c}^{\circ}=\frac{1}{2}\left(\mathcal{A}_{111}+\mathcal{A}_{122}\right)$ \\
\hline$Y^{3,1 s}=\frac{3}{2} S\left(1-5 C^{2}\right) \sin \phi$ & $\mathcal{A}_{1 s}^{\circ}=\frac{1}{2}\left(\mathcal{A}_{112}+\mathcal{A}_{222}\right)$ \\
\hline$Y^{3,2 c}=3 S^{2} C \cos 2 \phi$ & $\mathcal{A}_{2 c}^{\circ}=\frac{1}{2}\left(\mathcal{A}_{113}-\mathcal{A}_{223}\right)$ \\
\hline$Y^{3,2 s}=3 S^{2} C \sin 2 \phi$ & $\mathcal{A}_{2 s}^{\circ}=\mathcal{A}_{123}$ \\
\hline$Y^{3,3 c}=S^{3} \cos 3 \phi$ & $\mathcal{A}_{3 c}^{\circ}=\frac{1}{4}\left(\mathcal{A}_{111}-3 \mathcal{A}_{122}\right)$ \\
\hline$Y^{3,3 s}=S^{3} \sin 3 \phi$ & $\mathcal{A}_{3 s}^{\circ}=\frac{1}{4}\left(3 \mathcal{A}_{112}-\mathcal{A}_{222}\right)$ \\
\hline$Y^{4,0}=\frac{1}{2}\left(3-30 C^{2}+35 C^{4}\right)$ & $\mathcal{A}_{0}^{\mathrm{h}}=\frac{1}{4}\left(\mathcal{A}_{1111}+2 \mathcal{A}_{1122}+\mathcal{A}_{2222}\right)$ \\
\hline$Y^{4,1 c}=2 S C\left(3-7 C^{2}\right) \cos \phi$ & $\mathcal{A}_{1 c}^{\mathrm{h}}=\frac{1}{2}\left(\mathcal{A}_{1113}+\mathcal{A}_{1223}\right)$ \\
\hline$Y^{4,1 s}=2 S C\left(3-7 C^{2}\right) \sin \phi$ & $\mathcal{A}_{1 s}^{\mathrm{h}}=\frac{1}{2}\left(\mathcal{A}_{1123}+\mathcal{A}_{2223}\right)$ \\
\hline$Y^{4,2 c}=S^{2}\left(1-7 C^{2}\right) \cos 2 \phi$ & $\mathcal{A}_{2 c}^{\mathrm{h}}=\frac{1}{2}\left(\mathcal{A}_{1111}-\mathcal{A}_{2222}\right)$ \\
\hline$Y^{4,2 s}=S^{2}\left(1-7 C^{2}\right) \sin 2 \phi$ & $\mathcal{A}_{2 s}^{\mathrm{h}}=\mathcal{A}_{1112}+\mathcal{A}_{1222}$ \\
\hline$Y^{4,3 c}=4 S^{3} C \cos 3 \phi$ & $\mathcal{A}_{3 c}^{\mathrm{h}}=\frac{1}{4}\left(\mathcal{A}_{1113}-3 \mathcal{A}_{1223}\right)$ \\
\hline$Y^{4,3 s}=4 S^{3} C \sin 3 \phi$ & $\mathcal{A}_{3 s}^{\mathrm{h}}=\frac{1}{4}\left(3 \mathcal{A}_{1123}-\mathcal{A}_{2223}\right)$ \\
\hline$Y^{4,4 c}=S^{4} \cos 4 \phi$ & $\mathcal{A}_{4 c}^{\mathrm{h}}=\frac{1}{8}\left(\mathcal{A}_{1111}-6 \mathcal{A}_{1122}+\mathcal{A}_{2222}\right)$ \\
\hline$Y^{4,4 s}=S^{4} \sin 4 \phi$ & $\mathcal{A}_{4 s}^{\mathrm{h}}=\frac{1}{2}\left(\mathcal{A}_{1112}-\mathcal{A}_{1222}\right)$ \\
\hline
\end{tabular}


TABLE III. The first column lists the harmonic components of type- $\mathcal{E}$, even-parity tidal potentials, as defined in Table 1 . The second column lists their expansions in scalar, vector, and tensor harmonics. The components of $\mathcal{E}^{\text {h }}$ come with an additional factor of 2 to accommodate Zhang's choice of normalization; see Table 1 .

\begin{tabular}{|c|c|}
\hline $\begin{array}{l}\mathcal{E}_{0}^{\mathrm{q}}=\frac{1}{2}\left(\mathcal{E}_{11}+\mathcal{E}_{22}\right) \\
\mathcal{E}_{1 c}^{\mathrm{q}}=\mathcal{E}_{13} \\
\mathcal{E}_{1 s}^{\mathrm{q}}=\mathcal{E}_{23} \\
\mathcal{E}_{2 c}^{\mathrm{q}}=\frac{1}{2}\left(\mathcal{E}_{11}-\mathcal{E}_{22}\right) \\
\mathcal{E}_{2 s}^{\mathrm{q}}=\mathcal{E}_{12}\end{array}$ & $\begin{array}{l}\mathcal{E}^{q}=\sum_{m} \mathcal{E}_{m}^{q} Y^{2, m} \\
\mathcal{E}_{A}^{q}=\frac{1}{2} \sum_{m} \mathcal{E}_{m}^{q} Y_{A}^{2, m} \\
\mathcal{E}_{A B}^{q}=\sum_{m} \mathcal{E}_{m}^{q} Y_{A B}^{2, m}\end{array}$ \\
\hline $\begin{array}{l}\mathcal{E}_{0}^{\circ}=\frac{1}{2}\left(\mathcal{E}_{113}+\mathcal{E}_{223}\right) \\
\mathcal{E}_{1 c}^{\circ}=\frac{1}{2}\left(\mathcal{E}_{111}+\mathcal{E}_{122}\right) \\
\mathcal{E}_{1 s}^{\circ}=\frac{1}{2}\left(\mathcal{E}_{112}+\mathcal{E}_{222}\right) \\
\mathcal{E}_{2 c}^{\circ}=\frac{1}{2}\left(\mathcal{E}_{113}-\mathcal{E}_{223}\right) \\
\mathcal{E}_{2 s}^{\circ}=\mathcal{E}_{123} \\
\mathcal{E}_{3 c}^{\circ}=\frac{1}{4}\left(\mathcal{E}_{111}-3 \mathcal{E}_{122}\right) \\
\mathcal{E}_{3 s}^{\circ}=\frac{1}{4}\left(3 \mathcal{E}_{112}-\mathcal{E}_{222}\right)\end{array}$ & $\begin{array}{l}\mathcal{E}^{\circ}=\sum_{m} \mathcal{E}_{m}^{\circ} Y^{3, m} \\
\mathcal{E}_{A}^{\circ}=\frac{1}{3} \sum_{m} \mathcal{E}_{m}^{\circ} Y_{A}^{3, m} \\
\mathcal{E}_{A B}^{\circ}=\frac{1}{3} \sum_{m} \mathcal{E}_{m}^{\circ} Y_{A B}^{3, m}\end{array}$ \\
\hline $\begin{array}{l}\mathcal{E}_{0}^{\mathrm{h}}=\frac{1}{2}\left(\mathcal{E}_{1111}+2 \mathcal{E}_{1122}+\mathcal{E}_{2222}\right) \\
\mathcal{E}_{1 c}^{\mathrm{h}}=\mathcal{E}_{1113}+\mathcal{E}_{1223} \\
\mathcal{E}_{1 s}^{\mathrm{h}}=\mathcal{E}_{1123}+\mathcal{E}_{2223} \\
\mathcal{E}_{2 c}^{\mathrm{h}}=\mathcal{E}_{1111}-\mathcal{E}_{2222} \\
\mathcal{E}_{2 s}^{\mathrm{h}}=2\left(\mathcal{E}_{1112}+\mathcal{E}_{1222}\right) \\
\mathcal{E}_{3 c}^{\mathrm{h}}=\frac{1}{2}\left(\mathcal{E}_{1113}-3 \mathcal{E}_{1223}\right) \\
\mathcal{E}_{3 s}^{\mathrm{h}}=\frac{1}{2}\left(3 \mathcal{E}_{1123}-\mathcal{E}_{2223}\right) \\
\mathcal{E}_{4 c}^{\mathrm{h}}=\frac{1}{4}\left(\mathcal{E}_{1111}-6 \mathcal{E}_{1122}+\mathcal{E}_{2222}\right) \\
\mathcal{E}_{4 s}^{\mathrm{h}}=\mathcal{E}_{1112}-\mathcal{E}_{1222}\end{array}$ & $\begin{array}{l}\mathcal{E}^{\mathrm{h}}=\sum_{m} \mathcal{E}_{m}^{\mathrm{h}} Y^{4, m} \\
\mathcal{E}_{A}^{\mathrm{h}}=\frac{1}{4} \sum_{m} \mathcal{E}_{m}^{\mathrm{h}} Y_{A}^{4, m} \\
\mathcal{E}_{A B}^{\mathrm{h}}=\frac{1}{6} \sum_{m} \mathcal{E}_{m}^{\mathrm{h}} Y_{A B}^{4, m}\end{array}$ \\
\hline
\end{tabular}


TABLE IV. The first column lists the harmonic components of type- $\mathcal{B}$, odd-parity tidal potentials, as defined in Table 1 The second column lists their expansions in scalar, vector, and tensor harmonics. The components of $\mathcal{B}^{\circ}$ come with an additional factor of $\frac{4}{3}$ to accommodate Zhang's choice of normalization, and those of $\mathcal{B}^{\text {h }}$ come with an additional factor $\frac{10}{3}$; see Table $\mathrm{I}$

\begin{tabular}{|c|c|}
\hline \multicolumn{2}{|l|}{$\mathcal{B}_{0}^{\mathrm{q}}=\frac{1}{2}\left(\mathcal{B}_{11}+\mathcal{B}_{22}\right)$} \\
\hline $\mathcal{B}_{1 c}^{\mathrm{q}}=\mathcal{B}_{13}$ & $\mathcal{B}^{q}=\sum_{m} \mathcal{B}_{m}^{q} Y^{2, m}$ \\
\hline $\mathcal{B}_{1 s}^{\mathrm{q}}=\mathcal{B}_{23}$ & $\mathcal{B}_{A}^{\mathrm{q}}=\frac{1}{2} \sum_{m} \mathcal{B}_{m}^{\mathrm{q}} X_{A}^{2, m}$ \\
\hline $\mathcal{B}_{2 c}^{q}=\frac{1}{2}\left(\mathcal{B}_{11}-\mathcal{B}_{22}\right)$ & $\mathcal{B}_{A B}^{\mathrm{q}}=\sum_{m} \mathcal{B}_{m}^{\mathrm{q}} X_{A B}^{2, m}$ \\
\hline $\mathcal{B}_{2 s}^{\mathrm{q}}=\mathcal{B}_{12}$ & \\
\hline \multicolumn{2}{|l|}{$\mathcal{B}_{0}^{\circ}=\frac{2}{3}\left(\mathcal{B}_{113}+\mathcal{B}_{223}\right)$} \\
\hline \multicolumn{2}{|l|}{$\mathcal{B}_{1 c}^{\circ}=\frac{2}{3}\left(\mathcal{B}_{111}+\mathcal{B}_{122}\right)$} \\
\hline $\mathcal{B}_{1 s}^{\circ}=\frac{2}{3}\left(\mathcal{B}_{112}+\mathcal{B}_{222}\right)$ & $\mathcal{B}^{\circ}=\sum_{m} \mathcal{B}_{m}^{\circ} Y^{3, m}$ \\
\hline $\mathcal{B}_{2 c}^{\circ}=\frac{2}{3}\left(\mathcal{B}_{113}-\mathcal{B}_{223}\right)$ & $\mathcal{B}_{A}^{\circ}=\frac{1}{3} \sum_{m} \mathcal{B}_{m}^{\circ} X_{A}^{3, m}$ \\
\hline $\mathcal{B}_{2 s}^{\circ}=\frac{4}{3} \mathcal{B}_{123}$ & $\mathcal{B}_{A B}^{\circ}=\frac{1}{3} \sum_{m} \mathcal{B}_{m}^{\circ} X_{A B}^{3, m}$ \\
\hline \multicolumn{2}{|l|}{$\mathcal{B}_{3 c}^{\circ}=\frac{1}{3}\left(\mathcal{B}_{111}-3 \mathcal{B}_{122}\right)$} \\
\hline \multicolumn{2}{|l|}{$\mathcal{B}_{3 s}^{\circ}=\frac{1}{3}\left(3 \mathcal{B}_{112}-\mathcal{B}_{222}\right)$} \\
\hline \multicolumn{2}{|l|}{$\mathcal{B}_{0}^{\mathrm{h}}=\frac{5}{6}\left(\mathcal{B}_{1111}+2 \mathcal{B}_{1122}+\mathcal{B}_{2222}\right)$} \\
\hline \multicolumn{2}{|l|}{$\mathcal{B}_{1 c}^{\mathrm{h}}=\frac{5}{3}\left(\mathcal{B}_{1113}+\mathcal{B}_{1223}\right)$} \\
\hline \multicolumn{2}{|l|}{$\mathcal{B}_{1 s}^{\mathrm{h}}=\frac{5}{3}\left(\mathcal{B}_{1123}+\mathcal{B}_{2223}\right)$} \\
\hline $\mathcal{B}_{2 c}^{\mathrm{h}}=\frac{5}{3}\left(\mathcal{B}_{1111}-\mathcal{B}_{2222}\right)$ & $\mathcal{B}^{\mathrm{h}}=\sum_{m} \mathcal{B}_{m}^{\mathrm{h}} Y^{4, m}$ \\
\hline $\mathcal{B}_{2 s}^{\mathrm{h}}=\frac{10}{3}\left(\mathcal{B}_{1112}+\mathcal{B}_{1222}\right)$ & $\mathcal{B}_{A}^{\mathrm{h}}=\frac{1}{4} \sum_{m} \mathcal{B}_{m}^{\mathrm{h}} X_{A}^{4, m}$ \\
\hline $\mathcal{B}_{3 c}^{\mathrm{h}}=\frac{5}{6}\left(\mathcal{B}_{1113}-3 \mathcal{B}_{1223}\right)$ & $\mathcal{B}_{A B}^{\mathrm{h}}=\frac{1}{6} \sum_{m} \mathcal{B}_{m}^{\mathrm{h}} X_{A B}^{4, m}$ \\
\hline \multicolumn{2}{|l|}{$\mathcal{B}_{3 s}^{\mathrm{h}}=\frac{5}{6}\left(3 \mathcal{B}_{1123}-\mathcal{B}_{2223}\right)$} \\
\hline \multicolumn{2}{|l|}{$\mathcal{B}_{4 c}^{\mathrm{h}}=\frac{5}{12}\left(\mathcal{B}_{1111}-6 \mathcal{B}_{1122}+\mathcal{B}_{2222}\right)$} \\
\hline $\mathcal{B}_{4 s}^{\mathrm{h}}=\frac{5}{3}\left(\mathcal{B}_{1112}-\mathcal{B}_{1222}\right)$ & \\
\hline
\end{tabular}

TABLE V. Harmonic components of type- $\mathcal{E} \mathcal{E}$, even-parity tidal potentials, as defined in Table I] The spherical-harmonic decompositions are $\mathcal{P}^{\mathrm{q}}=\sum_{m} \mathcal{P}_{m}^{\mathrm{q}} Y^{2, m}, \mathcal{P}_{A}^{\mathrm{q}}=\frac{1}{2} \sum_{m} \mathcal{P}_{m}^{\mathrm{q}} Y_{A}^{2, m}, \mathcal{P}_{A B}^{\mathrm{q}}=\sum_{m} \mathcal{P}_{m}^{\mathrm{q}} Y_{A B}^{2, m}, \mathcal{P}^{\mathrm{h}}=\sum_{m} \mathcal{P}_{m}^{\mathrm{h}} Y^{4, m}, \mathcal{P}_{A}^{\mathrm{h}}=\frac{1}{4} \sum_{m} \mathcal{P}_{m}^{\mathrm{h}} Y_{A}^{4, m}$, and $\mathcal{P}_{A B}^{\mathrm{h}}=\frac{1}{6} \sum_{m} \mathcal{P}_{m}^{\mathrm{h}} Y_{A B}^{4, m}$.

$$
\begin{aligned}
& \mathcal{P}^{\mathrm{m}}=6\left(\mathcal{E}_{0}^{\mathrm{q}}\right)^{2}+2\left(\mathcal{E}_{1 c}^{\mathrm{q}}\right)^{2}+2\left(\mathcal{E}_{1 s}^{\mathrm{q}}\right)^{2}+2\left(\mathcal{E}_{2 c}^{\mathrm{q}}\right)^{2}+2\left(\mathcal{E}_{2 s}^{\mathrm{q}}\right)^{2} \\
& \mathcal{P}_{0}^{\mathrm{q}}=-\left(\mathcal{E}_{0}^{\mathrm{q}}\right)^{2}-\frac{1}{6}\left(\mathcal{E}_{1 c}^{\mathrm{q}}\right)^{2}-\frac{1}{6}\left(\mathcal{E}_{1 s}^{\mathrm{q}}\right)^{2}+\frac{1}{3}\left(\mathcal{E}_{2 c}^{\mathrm{q}}\right)^{2}+\frac{1}{3}\left(\mathcal{E}_{2 s}^{\mathrm{q}}\right)^{2} \\
& \mathcal{P}_{1 c}^{\mathrm{q}}=-\mathcal{E}_{0}^{\mathrm{q}} \mathcal{E}_{1 c}^{\mathrm{q}}+\mathcal{E}_{1 c}^{\mathrm{q}} \mathcal{E}_{2 c}^{\mathrm{q}}+\mathcal{E}_{1 s}^{\mathrm{q}} \mathcal{E}_{2 s}^{\mathrm{q}} \\
& \mathcal{P}_{1 s}^{\mathrm{q}}=-\mathcal{E}_{0}^{\mathrm{q}} \mathcal{E}_{1 s}^{\mathrm{q}}+\mathcal{E}_{1 c}^{\mathrm{q}} \mathcal{E}_{2 s}^{\mathrm{q}}-\mathcal{E}_{1 s}^{\mathrm{q}} \mathcal{E}_{2 c}^{\mathrm{q}} \\
& \mathcal{P}_{2 c}^{\mathrm{q}}=2 \mathcal{E}_{0}^{\mathrm{q}} \mathcal{E}_{2 c}^{\mathrm{q}}+\frac{1}{2}\left(\mathcal{E}_{1 c}^{\mathrm{q}}\right)^{2}-\frac{1}{2}\left(\mathcal{E}_{1 s}^{\mathrm{q}}\right)^{2} \\
& \mathcal{P}_{2 s}^{\mathrm{q}}=2 \mathcal{E}_{0}^{\mathrm{q}} \mathcal{E}_{2 s}^{\mathrm{q}}+\mathcal{E}_{1 c}^{\mathrm{q}} \mathcal{E}_{1 s}^{\mathrm{q}} \\
& \mathcal{P}_{0}^{\mathrm{h}}=\frac{18}{35}\left(\mathcal{E}_{0}^{\mathrm{q}}\right)^{2}-\frac{4}{35}\left(\mathcal{E}_{1 c}^{\mathrm{q}}\right)^{2}-\frac{4}{35}\left(\mathcal{E}_{1 s}^{\mathrm{q}}\right)^{2}+\frac{1}{35}\left(\mathcal{E}_{2 c}^{\mathrm{q}}\right)^{2}+\frac{1}{35}\left(\mathcal{E}_{2 s}^{\mathrm{q}}\right)^{2} \\
& \mathcal{P}_{1 c}^{\mathrm{h}}=\frac{6}{7} \mathcal{E}_{0}^{\mathrm{q}} \mathcal{E}_{1 c}^{\mathrm{q}}+\frac{1}{7} \mathcal{E}_{1 c}^{\mathrm{q}} \mathcal{E}_{2 c}^{\mathrm{q}}+\frac{1}{7} \mathcal{E}_{1 s}^{\mathrm{q}} \mathcal{E}_{2 s}^{\mathrm{q}} \\
& \mathcal{P}_{1 s}^{\mathrm{h}}=\frac{6}{7} \mathcal{E}_{0}^{\mathrm{q}} \mathcal{E}_{1 s}^{\mathrm{q}}+\frac{1}{7} \mathcal{E}_{1 c}^{\mathrm{q}} \mathcal{E}_{2 s}^{\mathrm{q}}-\frac{1}{7} \mathcal{E}_{1 s}^{\mathrm{q}} \mathcal{E}_{2 c}^{\mathrm{q}} \\
& \mathcal{P}_{2 c}^{\mathrm{h}}=\frac{6}{7} \mathcal{E}_{0}^{\mathrm{q}} \mathcal{E}_{2 c}^{\mathrm{q}}-\frac{2}{7}\left(\mathcal{E}_{1 c}^{\mathrm{q}}\right)^{2}+\frac{2}{7}\left(\mathcal{E}_{1 s}^{\mathrm{q}}\right)^{2} \\
& \mathcal{P}_{2 s}^{\mathrm{h}}=\frac{6}{7} \mathcal{E}_{0}^{\mathrm{q}} \mathcal{E}_{2 s}^{\mathrm{q}}-\frac{4}{7} \mathcal{E}_{1 c}^{\mathrm{q}} \mathcal{E}_{1 s}^{\mathrm{q}} \\
& \mathcal{P}_{3 c}^{\mathrm{h}}=\frac{1}{2} \mathcal{E}_{1 c}^{\mathrm{q}} \mathcal{E}_{2 c}^{\mathrm{q}}-\frac{1}{2} \mathcal{E}_{1 s}^{\mathrm{q}} \mathcal{E}_{2 s}^{\mathrm{q}} \\
& \mathcal{P}_{3 s}^{\mathrm{h}}=\frac{1}{2} \mathcal{E}_{1 c}^{\mathrm{q}} \mathcal{E}_{2 s}^{\mathrm{q}}+\frac{1}{2} \mathcal{E}_{1 s}^{\mathrm{q}} \mathcal{E}_{2 c}^{\mathrm{q}} \\
& \mathcal{P}_{4 c}^{\mathrm{h}}=\frac{1}{2}\left(\mathcal{E}_{2 c}^{\mathrm{q}}\right)^{2}-\frac{1}{2}\left(\mathcal{E}_{2 s}^{\mathrm{q}}\right)^{2} \\
& \mathcal{P}_{4 s}^{\mathrm{h}}=\mathcal{E}_{2 c}^{\mathrm{q}} \mathcal{E}_{2 s}^{\mathrm{q}}
\end{aligned}
$$


TABLE VI. Harmonic components of type- $\mathcal{B B}$, even-parity tidal potentials, as defined in Table I The spherical-harmonic decompositions are $\mathcal{Q}^{\mathrm{q}}=\sum_{m} \mathcal{Q}_{m}^{\mathrm{q}} Y^{2, m}, \mathcal{Q}_{A}^{\mathrm{q}}=\frac{1}{2} \sum_{m} \mathcal{Q}_{m}^{\mathrm{q}} Y_{A}^{2, m}, \mathcal{Q}_{A B}^{\mathrm{q}}=\sum_{m} \mathcal{Q}_{m}^{\mathrm{q}} Y_{A B}^{2, m}, \mathcal{Q}^{\mathrm{h}}=\sum_{m} \mathcal{Q}_{m}^{\mathrm{h}} Y^{4, m}, \mathcal{Q}_{A}^{\mathrm{h}}=\frac{1}{4} \sum_{m} \mathcal{Q}_{m}^{\mathrm{h}} Y_{A}^{4, m}$, and $\mathcal{Q}_{A B}^{\mathrm{h}}=\frac{1}{6} \sum_{m} \mathcal{Q}_{m}^{\mathrm{h}} Y_{A B}^{4, m}$.

$$
\begin{aligned}
& \mathcal{Q}^{\mathrm{m}}=6\left(\mathcal{B}_{0}^{\mathrm{q}}\right)^{2}+2\left(\mathcal{B}_{1 c}^{\mathrm{q}}\right)^{2}+2\left(\mathcal{B}_{1 s}^{\mathrm{q}}\right)^{2}+2\left(\mathcal{B}_{2 c}^{\mathrm{q}}\right)^{2}+2\left(\mathcal{B}_{2 s}^{\mathrm{q}}\right)^{2} \\
& \mathcal{Q}_{0}^{\mathrm{q}}=-\left(\mathcal{B}_{0}^{\mathrm{q}}\right)^{2}-\frac{1}{6}\left(\mathcal{B}_{1 c}^{\mathrm{q}}\right)^{2}-\frac{1}{6}\left(\mathcal{B}_{1 s}^{\mathrm{q}}\right)^{2}+\frac{1}{3}\left(\mathcal{B}_{2 c}^{\mathrm{q}}\right)^{2}+\frac{1}{3}\left(\mathcal{B}_{2 s}^{\mathrm{q}}\right)^{2} \\
& \mathcal{Q}_{1 c}^{\mathrm{q}}=-\mathcal{B}_{0}^{\mathrm{q}} \mathcal{B}_{1 c}^{\mathrm{q}}+\mathcal{B}_{1 c}^{\mathrm{q}} \mathcal{B}_{2 c}^{\mathrm{q}}+\mathcal{B}_{1 s}^{\mathrm{q}} \mathcal{B}_{2 s}^{\mathrm{q}} \\
& \mathcal{Q}_{1 s}^{\mathrm{q}}=-\mathcal{B}_{0}^{\mathrm{q}} \mathcal{B}_{1 s}^{\mathrm{q}}+\mathcal{B}_{1 c}^{\mathrm{q}} \mathcal{B}_{2 s}^{\mathrm{q}}-\mathcal{B}_{1 s}^{\mathrm{q}} \mathcal{B}_{2 c}^{\mathrm{q}} \\
& \mathcal{Q}_{2 c}^{\mathrm{q}}=2 \mathcal{B}_{0}^{\mathrm{q}} \mathcal{B}_{2 c}^{\mathrm{q}}+\frac{1}{2}\left(\mathcal{B}_{1 c}^{\mathrm{q}}\right)^{2}-\frac{1}{2}\left(\mathcal{B}_{1 s}^{\mathrm{q}}\right)^{2} \\
& \mathcal{Q}_{2 s}^{\mathrm{q}}=2 \mathcal{B}_{0}^{\mathrm{q}} \mathcal{B}_{2 s}^{\mathrm{q}}+\mathcal{B}_{1 c}^{\mathrm{q}} \mathcal{B}_{1 s}^{\mathrm{q}} \\
& \mathcal{Q}_{0}^{\mathrm{h}}=\frac{18}{35}\left(\mathcal{B}_{0}^{\mathrm{q}}\right)^{2}-\frac{4}{35}\left(\mathcal{B}_{1 c}^{\mathrm{q}}\right)^{2}-\frac{4}{35}\left(\mathcal{B}_{1 s}^{\mathrm{q}}\right)^{2}+\frac{1}{35}\left(\mathcal{B}_{2 c}^{\mathrm{q}}\right)^{2}+\frac{1}{35}\left(\mathcal{B}_{2 s}^{\mathrm{q}}\right)^{2} \\
& \mathcal{Q}_{1 c}^{\mathrm{h}}=\frac{6}{7} \mathcal{B}_{0}^{\mathrm{q}} \mathcal{B}_{1 c}^{\mathrm{q}}+\frac{1}{7} \mathcal{B}_{1 c}^{\mathrm{q}} \mathcal{B}_{2 c}^{\mathrm{q}}+\frac{1}{7} \mathcal{B}_{1 s}^{\mathrm{q}} \mathcal{B}_{2 s}^{\mathrm{q}} \\
& \mathcal{Q}_{1 s}^{\mathrm{h}}=\frac{6}{7} \mathcal{B}_{0}^{\mathrm{q}} \mathcal{B}_{1 s}^{\mathrm{q}}+\frac{1}{7} \mathcal{B}_{1 c}^{\mathrm{q}} \mathcal{B}_{2 s}^{\mathrm{q}}-\frac{1}{7} \mathcal{B}_{1 s}^{\mathrm{q}} \mathcal{B}_{2 c}^{\mathrm{q}} \\
& \mathcal{Q}_{2 c}^{\mathrm{h}}=\frac{6}{7} \mathcal{B}_{0}^{\mathrm{q}} \mathcal{B}_{2 c}^{\mathrm{q}}-\frac{2}{7}\left(\mathcal{B}_{1 c}^{\mathrm{q}}\right)^{2}+\frac{2}{7}\left(\mathcal{B}_{1 s}^{\mathrm{q}}\right)^{2} \\
& \mathcal{Q}_{2 s}^{\mathrm{h}}=\frac{6}{7} \mathcal{B}_{0}^{\mathrm{q}} \mathcal{B}_{2 s}^{\mathrm{q}}-\frac{4}{7} \mathcal{B}_{1 c}^{\mathrm{q}} \mathcal{B}_{1 s}^{\mathrm{q}} \\
& \mathcal{Q}_{3 c}^{\mathrm{h}}=\frac{1}{2} \mathcal{B}_{1 c}^{\mathrm{q}} \mathcal{B}_{2 c}^{\mathrm{q}}-\frac{1}{2} \mathcal{B}_{1 s}^{\mathrm{q}} \mathcal{B}_{2 s}^{\mathrm{q}} \\
& \mathcal{Q}_{3 s}^{\mathrm{h}}=\frac{1}{2} \mathcal{B}_{1 c}^{\mathrm{q}} \mathcal{B}_{2 s}^{\mathrm{q}}+\frac{1}{2} \mathcal{B}_{1 s}^{\mathrm{q}} \mathcal{B}_{2 c}^{\mathrm{q}} \\
& \mathcal{Q}_{4 c}^{\mathrm{h}}=\frac{1}{2}\left(\mathcal{B}_{2 c}^{\mathrm{q}}\right)^{2}-\frac{1}{2}\left(\mathcal{B}_{2 s}^{\mathrm{q}}\right)^{2} \\
& \mathcal{Q}_{4 s}^{\mathrm{h}}=\mathcal{B}_{2 c}^{\mathrm{q}} \mathcal{B}_{2 s}^{\mathrm{q}}
\end{aligned}
$$

TABLE VII. Harmonic components of type- $\mathcal{E} \mathcal{B}$, even-parity tidal potentials, as defined in Table I] The spherical-harmonic decompositions are $\mathcal{G}^{\mathrm{d}}=\sum_{m} \mathcal{G}_{m}^{\mathrm{d}} Y^{1, m}, \mathcal{G}_{A}^{\mathrm{d}}=\sum_{m} \mathcal{G}_{m}^{\mathrm{d}} Y_{A}^{1, m}, \mathcal{G}^{\circ}=\sum_{m} \mathcal{G}_{m}^{\circ} Y^{3, m}, \mathcal{G}_{A}^{\circ}=\frac{1}{3} \sum_{m} \mathcal{G}_{m}^{\circ} Y_{A}^{3, m}$, and $\mathcal{G}_{A B}^{\circ}=\frac{1}{3} \sum_{m} \mathcal{G}_{m}^{\circ} Y_{A B}^{3, m}$.

$$
\begin{aligned}
& \mathcal{G}_{0}^{\mathrm{d}}=\mathcal{E}_{1 c}^{\mathrm{q}} \mathcal{B}_{1 s}^{\mathrm{q}}-\mathcal{E}_{1 s}^{\mathrm{q}} \mathcal{B}_{1 c}^{\mathrm{q}}+2 \mathcal{E}_{2 c}^{\mathrm{q}} \mathcal{B}_{2 s}^{\mathrm{q}}-2 \mathcal{E}_{2 s}^{\mathrm{q}} \mathcal{B}_{2 c}^{\mathrm{q}} \\
& \mathcal{G}_{1 c}^{\mathrm{d}}=\left(3 \mathcal{E}_{0}^{\mathrm{q}}-\mathcal{E}_{2 c}^{\mathrm{q}}\right) \mathcal{B}_{1 s}^{\mathrm{q}}-\mathcal{E}_{1 c}^{\mathrm{q}} \mathcal{B}_{2 s}^{\mathrm{q}}-\mathcal{E}_{1 s}^{\mathrm{q}}\left(3 \mathcal{B}_{0}^{\mathrm{q}}-\mathcal{B}_{2 c}^{\mathrm{q}}\right)+\mathcal{E}_{2 s}^{\mathrm{q}} \mathcal{B}_{1 c}^{\mathrm{q}} \\
& \mathcal{G}_{1 s}^{\mathrm{d}}=-\left(3 \mathcal{E}_{0}^{\mathrm{q}}+\mathcal{E}_{2 c}^{\mathrm{q}}\right) \mathcal{B}_{1 c}^{\mathrm{q}}+\mathcal{E}_{1 c}^{\mathrm{q}}\left(3 \mathcal{B}_{0}^{\mathrm{q}}+\mathcal{B}_{2 c}^{\mathrm{q}}\right)+\mathcal{E}_{1 s}^{\mathrm{q}} \mathcal{B}_{2 s}^{\mathrm{q}}-\mathcal{E}_{2 s}^{\mathrm{q}} \mathcal{B}_{1 s}^{\mathrm{q}} \\
& \mathcal{G}_{0}^{\circ}=-\frac{2}{5} \mathcal{E}_{1 c}^{\mathrm{q}} \mathcal{B}_{1 s}^{\mathrm{q}}+\frac{2}{5} \mathcal{E}_{1 s}^{\mathrm{q}} \mathcal{B}_{1 c}^{\mathrm{q}}+\frac{1}{5} \mathcal{E}_{2 c}^{\mathrm{q}} \mathcal{B}_{2 s}^{\mathrm{q}}-\frac{1}{5} \mathcal{E}_{2 s}^{\mathrm{q}} \mathcal{B}_{2 c}^{\mathrm{q}} \\
& \mathcal{G}_{1 c}^{\circ}=-\frac{2}{5} \mathcal{E}_{0}^{\mathrm{q}} \mathcal{B}_{1 s}^{\mathrm{q}}-\frac{1}{5} \mathcal{E}_{1 c}^{\mathrm{q}} \mathcal{B}_{2 s}^{\mathrm{q}}+\frac{1}{5} \mathcal{E}_{1 s}^{\mathrm{q}}\left(2 \mathcal{B}_{0}^{\mathrm{q}}+\mathcal{B}_{2 c}^{\mathrm{q}}\right)-\frac{1}{5} \mathcal{E}_{2 c}^{\mathrm{q}} \mathcal{B}_{1 s}^{\mathrm{q}}+\frac{1}{5} \mathcal{E}_{2 s}^{\mathrm{q}} \mathcal{B}_{1 c}^{\mathrm{q}} \\
& \mathcal{G}_{1 s}^{\circ}=\frac{2}{5} \mathcal{E}_{0}^{\mathrm{q}} \mathcal{B}_{1 c}^{\mathrm{q}}-\frac{1}{5} \mathcal{E}_{1 c}^{\mathrm{q}}\left(2 \mathcal{B}_{0}^{\mathrm{q}}-\mathcal{B}_{2 c}^{\mathrm{q}}\right)+\frac{1}{5} \mathcal{E}_{1 s}^{\mathrm{q}} \mathcal{B}_{2 s}^{\mathrm{q}}-\frac{1}{5} \mathcal{E}_{2 c}^{\mathrm{q}} \mathcal{B}_{1 c}^{\mathrm{q}}-\frac{1}{5} \mathcal{E}_{2 s}^{\mathrm{q}} \mathcal{B}_{1 s}^{\mathrm{q}} \\
& \mathcal{G}_{2 c}^{\circ}=\mathcal{E}_{0}^{\mathrm{q}} \mathcal{B}_{2 s}^{\mathrm{q}}-\mathcal{E}_{2 s}^{\mathrm{q}} \mathcal{B}_{0}^{\mathrm{q}} \\
& \mathcal{G}_{2 s}^{\circ}=-\mathcal{E}_{0}^{\mathrm{q}} \mathcal{B}_{2 c}^{\mathrm{q}}+\mathcal{E}_{2 c}^{\mathrm{q}} \mathcal{B}_{0}^{\mathrm{q}} \\
& \mathcal{G}_{3 c}^{\circ}=-\frac{1}{2} \mathcal{E}_{1 c}^{\mathrm{q}} \mathcal{B}_{2 s}^{\mathrm{q}}-\frac{1}{2} \mathcal{E}_{1 s}^{\mathrm{q}} \mathcal{B}_{2 c}^{\mathrm{q}}+\frac{1}{2} \mathcal{E}_{2 c}^{\mathrm{q}} \mathcal{B}_{1 s}^{\mathrm{q}}+\frac{1}{2} \mathcal{E}_{2 s}^{\mathrm{q}} \mathcal{B}_{1 c}^{\mathrm{q}} \\
& \mathcal{G}_{3 s}^{\circ}=\frac{1}{2} \mathcal{E}_{1 c}^{\mathrm{q}} \mathcal{B}_{2 c}^{\mathrm{q}}-\frac{1}{2} \mathcal{E}_{1 s}^{\mathrm{q}} \mathcal{B}_{2 s}^{\mathrm{q}}-\frac{1}{2} \mathcal{E}_{2 c}^{\mathrm{q}} \mathcal{B}_{1 c}^{\mathrm{q}}+\frac{1}{2} \mathcal{E}_{2 s}^{\mathrm{q}} \mathcal{B}_{1 s}^{\mathrm{q}}
\end{aligned}
$$


TABLE VIII. Harmonic components of type- $\mathcal{E B}$, odd-parity tidal potentials, as defined in Table I] The spherical-harmonic decompositions are $\mathcal{H}_{A}^{\mathrm{q}}=\frac{1}{2} \sum_{m} \mathcal{H}_{m}^{\mathrm{q}} X_{A}^{2, m}, \mathcal{H}_{A B}^{\mathrm{q}}=\sum_{m} \mathcal{H}_{m}^{\mathrm{q}} X_{A B}^{2, m}, \mathcal{H}_{A}^{\mathrm{h}}=\frac{1}{4} \sum_{m} \mathcal{H}_{m}^{\mathrm{h}} X_{A}^{4, m}$, and $\mathcal{H}_{A B}^{\mathrm{h}}=\frac{1}{6} \sum_{m} \mathcal{H}_{m}^{\mathrm{h}} X_{A B}^{4, m}$.

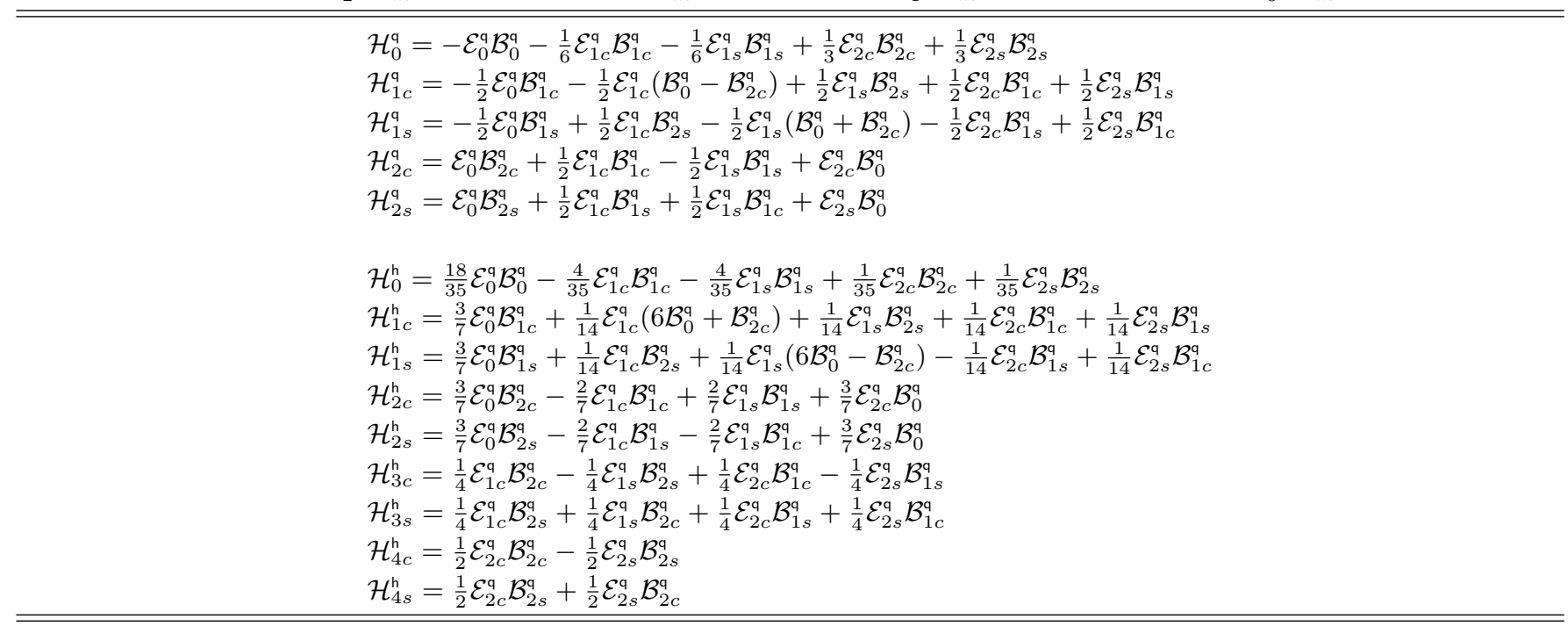


TABLE IX. Radial functions in $\left(v, r, \theta^{A}\right)$ coordinates: type- $\mathcal{E}$ potentials. The functions are expressed in terms of $x:=r / M$ and $f:=1-2 / x$. The dilogarithm function is defined as $\operatorname{dilog}(z)=\int_{1}^{z} d y \ln (y) /(1-y)$, with $\ln (z)$ denoting the natural logarithm. The constants $\mathrm{e}_{1}^{\mathrm{q}}, \mathrm{e}_{2}^{\mathrm{q}}$, and $\mathrm{e}_{1}^{\circ}$ are arbitrary constants of integration, whose meaning is explained in the text. In $\ddot{e}_{v v}^{q}$, the function $\dot{e}_{v v}^{q}$ is evaluated with $\mathrm{e}_{1}^{q}=0$; a similar statement holds for $\ddot{e}_{v r}^{q}, \ddot{e}_{r r}^{q}$, and $\ddot{e}^{q}$.

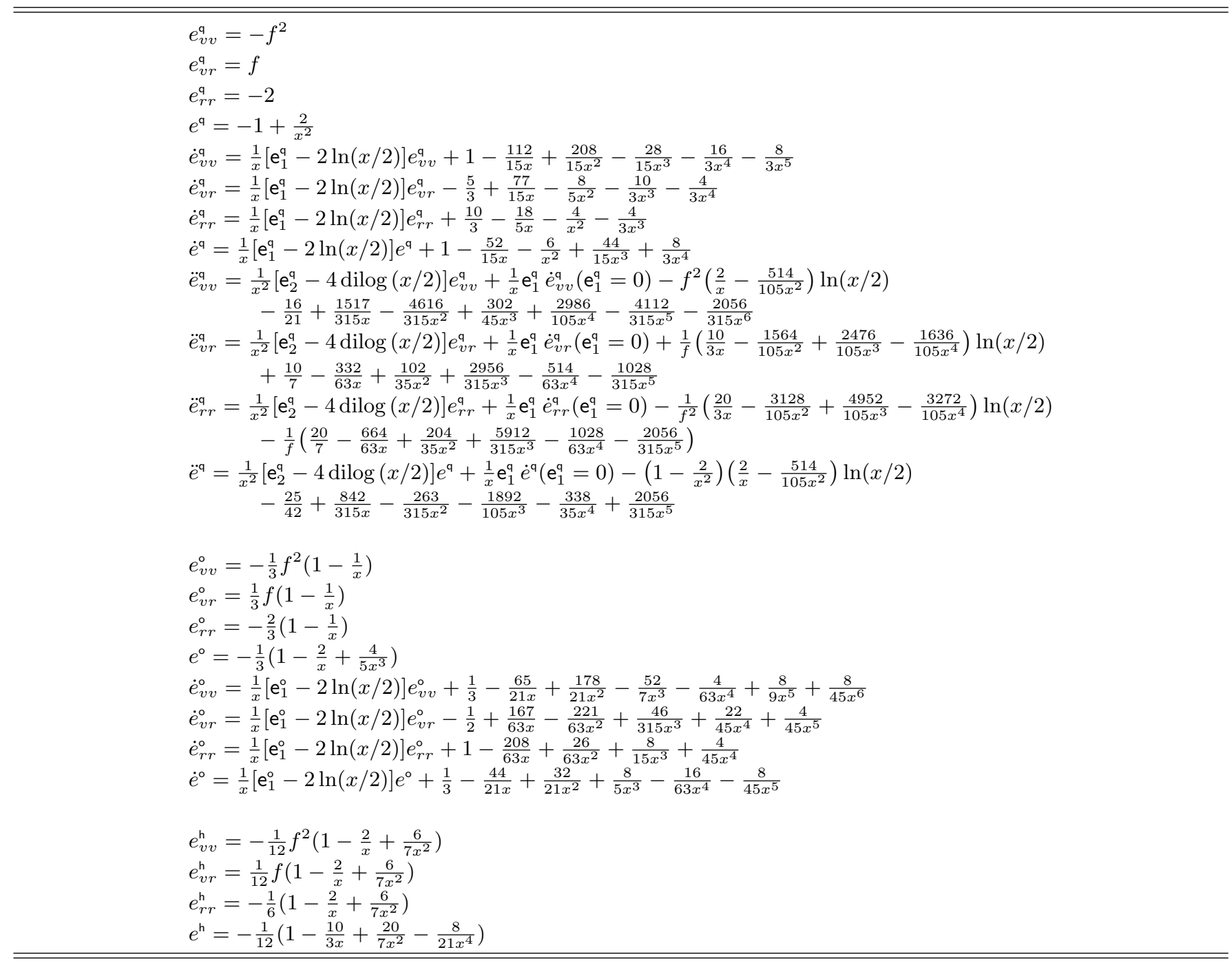


TABLE X. Radial functions in $\left(v, r, \theta^{A}\right)$ coordinates: type- $\mathcal{B}$ potentials. The functions are expressed in terms of $x:=r / M$ and $f:=1-2 / x$. The dilogarithm function is defined as $\operatorname{dilog}(z)=\int_{1}^{z} d y \ln (y) /(1-y)$, with $\ln (z)$ denoting the natural logarithm. The constants $b_{1}^{q}, b_{2}^{q}$, and $b_{1}^{\circ}$ are arbitrary constants of integration, whose meaning is explained in the text. In $\ddot{b}_{v}^{q}$, the function $\dot{b}_{v}^{q}$ is evaluated with $\mathrm{b}_{1}^{q}=0$; a similar statement holds for $\ddot{b}_{r}^{q}$.

$$
\begin{aligned}
& b_{v}^{q}=\frac{2}{3} f \\
& b_{r}^{\mathrm{q}}=-\frac{2}{3} \\
& \dot{b}_{v}^{q}=\frac{1}{x}\left[\mathrm{~b}_{1}^{\mathrm{q}}-2 \ln (x / 2)\right] b_{v}^{\mathrm{q}}-\frac{2}{3}+\frac{49}{15 x}-\frac{6}{5 x^{2}}-\frac{8}{3 x^{3}}-\frac{16}{9 x^{4}}-\frac{16}{9 x^{5}} \\
& \dot{b}_{r}^{\mathrm{q}}=\frac{1}{x}\left[\mathrm{~b}_{1}^{\mathrm{q}}-2 \ln (x / 2)\right] b_{r}^{\mathrm{q}}+\frac{5}{6}-\frac{8}{5 x}-\frac{2}{x^{2}}-\frac{4}{3 x^{3}}-\frac{8}{9 x^{4}} \\
& \ddot{b}_{v}^{q}=\frac{1}{x^{2}}\left[\mathrm{~b}_{2}^{\mathrm{q}}-4 \operatorname{dilog}(x / 2)\right] b_{v}^{\mathrm{q}}+\frac{1}{x} \mathrm{~b}_{1}^{\mathrm{q}} \dot{b}_{v}^{\mathrm{q}}\left(\mathrm{b}_{1}^{\mathrm{q}}=0\right)+f\left(\frac{4}{3 x}-\frac{158}{63 x^{2}}\right) \ln (x / 2) \\
& +\frac{17}{42}-\frac{1403}{630 x}+\frac{479}{126 x^{2}}+\frac{38}{7 x^{3}}-\frac{344}{63 x^{4}}-\frac{632}{189 x^{5}}-\frac{632}{189 x^{6}} \\
& \ddot{b}_{r}^{\mathrm{q}}=\frac{1}{x^{2}}\left[\mathrm{~b}_{2}^{\mathrm{q}}-4 \operatorname{dilog}(x / 2)\right] b_{r}^{\mathrm{q}}+\frac{1}{x} \mathrm{~b}_{1}^{\mathrm{q}} \dot{b}_{r}^{\mathrm{q}}\left(\mathrm{b}_{1}^{\mathrm{q}}=0\right)-\frac{1}{f}\left(\frac{5}{3 x}-\frac{326}{63 x^{2}}+\frac{316}{63 x^{3}}\right) \ln (x / 2) \\
& -\frac{4}{7}+\frac{395}{252 x}-\frac{100}{21 x^{3}}-\frac{200}{63 x^{4}}-\frac{316}{189 x^{5}} \\
& b_{v}^{\circ}=\frac{1}{4} f\left(1-\frac{4}{3 x}\right) \\
& b_{r}^{\circ}=-\frac{1}{4}\left(1-\frac{4}{3 x}\right) \\
& \dot{b}_{v}^{\circ}=\frac{1}{x}\left[\mathrm{~b}_{1}^{\circ}-2 \ln (x / 2)\right] b_{v}^{\circ}-\frac{1}{4}+\frac{1579}{840 x}-\frac{265}{84 x^{2}}+\frac{16}{35 x^{3}}+\frac{2}{3 x^{4}}+\frac{2}{9 x^{5}}+\frac{4}{45 x^{6}} \\
& \dot{b}_{r}^{\circ}=\frac{1}{x}\left[\mathrm{~b}_{1}^{\circ}-2 \ln (x / 2)\right] b_{r}^{\circ}+\frac{3}{10}-\frac{229}{168 x}+\frac{3}{7 x^{2}}+\frac{2}{5 x^{3}}+\frac{2}{15 x^{4}}+\frac{2}{45 x^{5}} \\
& b_{v}^{\mathrm{h}}=\frac{1}{15} f\left(1-\frac{5}{2 x}+\frac{10}{7 x^{2}}\right) \\
& b_{r}^{\mathrm{h}}=-\frac{1}{15}\left(1-\frac{5}{2 x}+\frac{10}{7 x^{2}}\right)
\end{aligned}
$$

TABLE XI. Radial functions in $\left(v, r, \theta^{A}\right)$ coordinates: type- $\mathcal{P}$ and type- $\mathcal{Q}$ potentials. The functions are expressed in terms of $x:=r / M$ and $f:=1-2 / x$. The constants $\mathrm{p}^{\mathrm{m}}, \mathrm{p}^{\mathrm{q}}, \mathrm{p}^{\mathrm{h}}, \mathrm{q}^{\mathrm{m}}, \mathrm{q}^{\mathrm{q}}$, and $\mathrm{q}^{\mathrm{h}}$ are arbitrary constants of integration, whose meaning is explained in the text.

\begin{tabular}{ll}
\hline \hline$p_{v v}^{\mathrm{m}}=\frac{2}{x^{5}} \mathrm{p}^{\mathrm{m}}-f^{2}\left(\frac{1}{15}+\frac{2}{75 x}-\frac{4}{25 x^{2}}+\frac{32}{225 x^{3}}+\frac{32}{225 x^{4}}\right)$ & $q_{v v}^{\mathrm{m}}=\frac{2}{x^{5}} \mathrm{q}^{\mathrm{m}}+f^{3}\left(\frac{1}{15}+\frac{2}{25 x}+\frac{2}{25 x^{2}}+\frac{4}{75 x^{3}}\right)$ \\
$p_{v r}^{\mathrm{m}}=-\frac{2}{x^{5} f} \mathrm{p}^{\mathrm{m}}+f\left(\frac{1}{15}+\frac{2}{75 x}-\frac{4}{25 x^{2}}+\frac{32}{225 x^{3}}+\frac{32}{225 x^{4}}\right)$ & $q_{v r}^{\mathrm{m}}=-\frac{2}{x^{5} f} \mathrm{q}^{\mathrm{m}}-f^{2}\left(\frac{1}{15}+\frac{2}{25 x}+\frac{2}{25 x^{2}}+\frac{4}{75 x^{3}}\right)$ \\
$p_{r r}^{\mathrm{m}}=\frac{4}{x^{5} f^{2}} \mathrm{p}^{\mathrm{m}}-2\left(\frac{1}{15}+\frac{2}{75 x}-\frac{4}{25 x^{2}}+\frac{32}{225 x^{3}}+\frac{32}{225 x^{4}}\right)$ & $q_{r r}^{\mathrm{m}}=\frac{4}{x^{5} f^{2}} \mathrm{q}^{\mathrm{m}}+2 f\left(\frac{1}{15}+\frac{2}{25 x}+\frac{2}{25 x^{2}}+\frac{4}{75 x^{3}}\right)$ \\
$p^{\mathrm{m}}=\frac{2}{75}-\frac{8}{45 x^{2}}+\frac{128}{225 x^{5}}$ & $q^{\mathrm{m}}=\frac{2}{225}-\frac{2}{45 x}+\frac{32}{75 x^{5}}$ \\
$p_{v v}^{\mathrm{q}}=\frac{1}{x^{2}} \mathrm{p}^{\mathrm{q}} e_{v v}^{\mathrm{q}}-f^{2}\left(\frac{2}{7}+\frac{6}{7 x}-\frac{8}{7 x^{2}}\right)$ & $q_{v v}^{\mathrm{q}}=\frac{1}{x^{2}} \mathrm{q}^{\mathrm{q}} e_{v v}^{\mathrm{q}}+f\left(\frac{2}{7}-\frac{2}{3 x}\right)$ \\
$p_{v r}^{\mathrm{q}}=\frac{1}{x^{2}} \mathrm{p}^{\mathrm{q}} e_{v r}^{\mathrm{q}}+f\left(\frac{2}{7}+\frac{6}{7 x}-\frac{8}{7 x^{2}}\right)$ & $q_{v r}^{\mathrm{q}}=\frac{1}{x^{2}} \mathrm{q}^{\mathrm{q}} e_{v r}^{\mathrm{q}}-\frac{2}{7}+\frac{2}{3 x}$ \\
$p_{r r}^{\mathrm{q}}=\frac{1}{x^{2}} \mathrm{p}^{\mathrm{q}} e_{r r}^{\mathrm{q}}-\frac{6}{7}-\frac{8}{7 x}+\frac{16}{7 x^{2}}$ & $q_{r r}^{\mathrm{q}}=\frac{1}{x^{2}} \mathrm{q}^{\mathrm{q}} e_{r r}^{\mathrm{q}}+\frac{34}{21}$ \\
$p^{\mathrm{q}}=\frac{1}{x^{2}} \mathrm{p}^{\mathrm{q}} e^{\mathrm{q}}-\frac{1}{14}+\frac{12}{7 x^{4}}$ & $q^{\mathrm{q}}=\frac{1}{x^{2}} \mathrm{q}^{\mathrm{q}} e^{\mathrm{q}}+\frac{1}{2}-\frac{4}{7 x}$ \\
$p_{v v}^{\mathrm{h}}=\mathrm{p}^{\mathrm{h}} e_{v v}^{\mathrm{h}}+f^{2}\left(\frac{367}{36}-\frac{164}{9 x}+\frac{7}{x^{2}}\right)$ & \\
$p_{v r}^{\mathrm{h}}=\mathrm{p}^{\mathrm{h}} e_{v r}^{\mathrm{h}}-f\left(\frac{367}{36}-\frac{164}{9 x}+\frac{7}{x^{2}}\right)$ & $q_{v v}^{\mathrm{h}}=\mathrm{q}^{\mathrm{h}} e_{v v}^{\mathrm{h}}-f\left(\frac{17}{36}-\frac{49}{54 x}\right)$ \\
$p_{r r}^{\mathrm{h}}=\mathrm{p}^{\mathrm{h}} e_{r r}^{\mathrm{h}}+\frac{379}{18}-\frac{340}{9 x}+\frac{14}{x^{2}}$ & $q_{v r}^{\mathrm{h}}=\mathrm{q}^{\mathrm{h}} e_{v r}^{\mathrm{h}}+\frac{17}{36}-\frac{49}{54 x}$ \\
$p^{\mathrm{h}}=\mathrm{p}^{\mathrm{h}} e^{\mathrm{h}}+\frac{391}{36}-\frac{868}{27 x}+\frac{206}{9 x^{2}}$ & $q_{r r}^{\mathrm{h}}=\mathrm{q}^{\mathrm{h}} e_{r r}^{\mathrm{h}}-\frac{29}{54}$ \\
\hline \hline & $q^{\mathrm{h}}=\mathrm{q}^{\mathrm{h}} e^{\mathrm{h}}-\frac{7}{108}+\frac{1}{27 x}$ \\
\hline
\end{tabular}


TABLE XII. Radial functions in $\left(v, r, \theta^{A}\right)$ coordinates: type- $\mathcal{G}$ and type- $\mathcal{H}$ potentials. The functions are expressed in terms of $x:=r / M$ and $f:=1-2 / x$. The constants $\mathrm{g}^{\circ}, \mathrm{h}^{\mathrm{q}}$, and $\mathrm{h}^{\mathrm{h}}$ are arbitrary constants of integration, whose meaning is explained in the text.

\begin{tabular}{ll}
\hline \hline$g_{v v}^{\mathrm{d}}=0$ & \\
$g_{v r}^{\mathrm{d}}=f\left(\frac{2}{15}-\frac{8}{15 x}\right)$ & $h_{v}^{\mathrm{q}}=\frac{1}{x^{2}} \mathrm{~h}^{\mathrm{q}} b_{v}^{\mathrm{q}}+f\left(\frac{4}{21}+\frac{16}{21 x}+\frac{32}{21 x^{2}}\right)$ \\
$g_{r r}^{\mathrm{d}}=-\frac{4}{15}+\frac{16}{15 x}$ & $h_{r}^{\mathrm{q}}=\frac{1}{x^{2}} \mathrm{~h}^{\mathrm{q}} b_{r}^{\mathrm{q}}-\left(\frac{4}{21}+\frac{16}{21 x}+\frac{32}{21 x^{2}}\right)$ \\
$g^{\mathrm{d}}=0$ & \\
& \\
$g_{v v}^{\mathrm{o}}=\frac{1}{x} \mathrm{~g}^{\mathrm{o}} e_{v v}^{\circ}$ & \\
$g_{v r}^{\circ}=\frac{1}{x} \mathrm{~g}^{\mathrm{o}} e_{v r}^{\circ}+f\left(\frac{1}{9}-\frac{4}{9 x}\right)$ & $h_{v}^{\mathrm{h}}=\mathrm{h}^{\mathrm{h}} b_{v}^{\mathrm{h}}-f\left(\frac{8}{3 x}-\frac{64}{21 x^{2}}\right)$ \\
$g_{r r}^{\circ}=\frac{1}{x} \mathrm{~g}^{\mathrm{o}} e_{r r}^{\circ}-2\left(\frac{1}{9}-\frac{4}{9 x}\right)$ & $h_{r}^{\mathrm{h}}=\mathrm{h}^{\mathrm{h}} b_{r}^{\mathrm{h}}+\frac{8}{3 x}-\frac{64}{21 x^{2}}$ \\
$g^{\circ}=\frac{1}{x} \mathrm{~g}^{\mathrm{o}} e^{\circ}$ & \\
\hline \hline
\end{tabular}

TABLE XIII. Radial functions in $\left(t, r, \theta^{A}\right)$ coordinates: type- $\mathcal{E}$ potentials. The functions are expressed in terms of $x:=r / M$ and $f:=1-2 / x$.

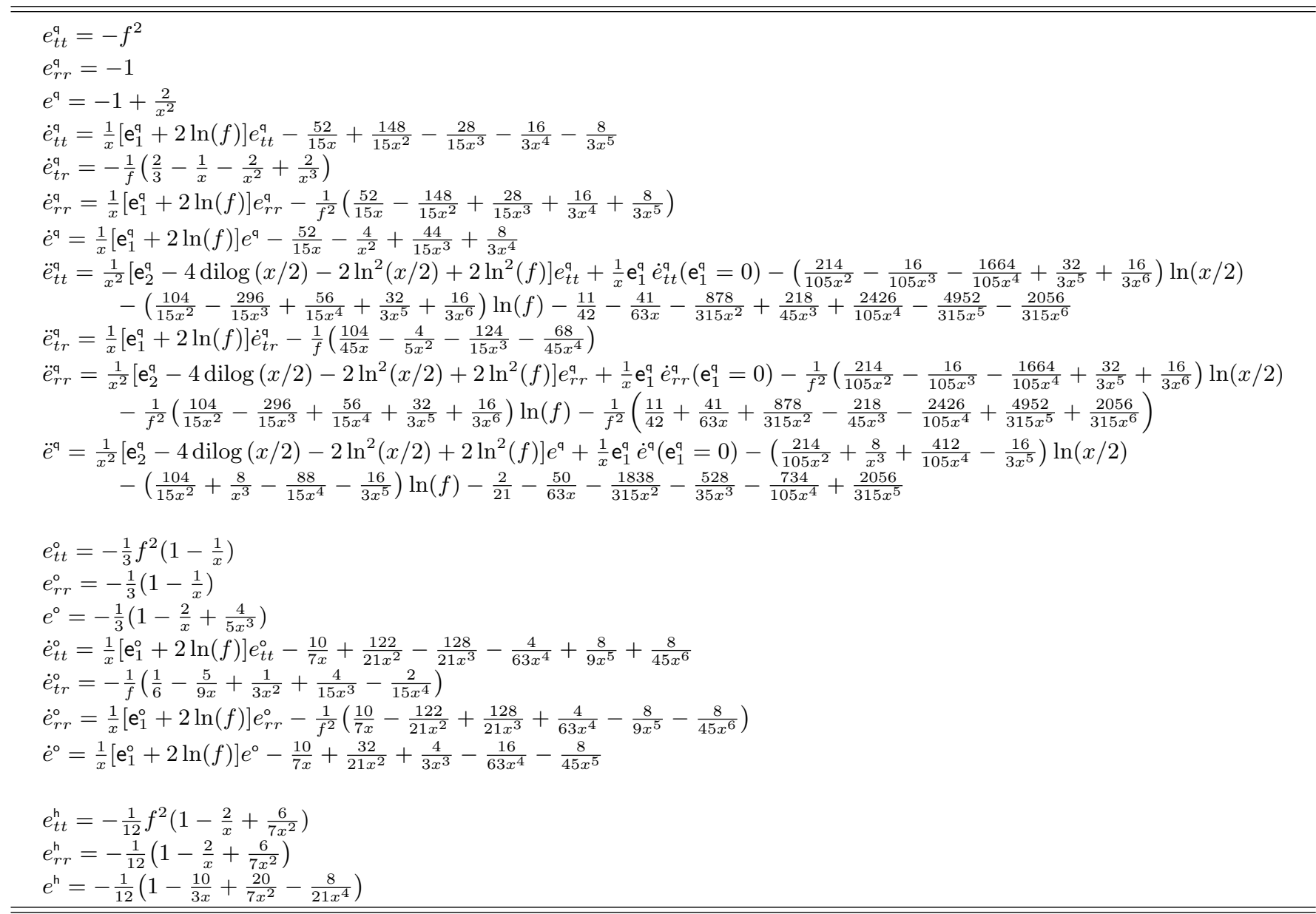


TABLE XIV. Radial functions in $\left(t, r, \theta^{A}\right)$ coordinates: type- $\mathcal{B}$ potentials. The functions are expressed in terms of $x:=r / M$ and $f:=1-2 / x$.

$$
\begin{aligned}
& b_{t}^{\mathrm{q}}=\frac{2}{3} f \\
& \dot{b}_{t}^{\mathrm{q}}=\frac{1}{x}\left[\mathrm{~b}_{1}^{\mathrm{q}}+2 \ln (f)\right] b_{t}^{\mathrm{q}}+\frac{29}{15 x}-\frac{6}{5 x^{2}}-\frac{8}{3 x^{3}}-\frac{16}{9 x^{4}}-\frac{16}{9 x^{5}} \\
& \dot{b}_{r}^{q}=\frac{1}{6 f} \\
& \ddot{b}_{t}^{q}=\frac{1}{x^{2}}\left[\mathrm{~b}_{2}^{q}-4 \operatorname{dilog}(x / 2)-2 \ln ^{2}(x / 2)+2 \ln ^{2}(f)\right] b_{t}^{q}+\frac{1}{x} \mathrm{~b}_{1}^{q} \dot{b}_{t}^{q}\left(\mathrm{~b}_{1}^{\mathrm{q}}=0\right)+\left(\frac{428}{315 x^{2}}+\frac{824}{315 x^{3}}-\frac{16}{3 x^{4}}-\frac{32}{9 x^{5}}-\frac{32}{9 x^{6}}\right) \ln (x / 2) \\
& +\left(\frac{58}{15 x^{2}}-\frac{12}{5 x^{3}}-\frac{16}{3 x^{4}}-\frac{32}{9 x^{5}}-\frac{32}{9 x^{6}}\right) \ln (f)+\frac{1}{14}+\frac{47}{126 x}+\frac{1639}{630 x^{2}}+\frac{58}{21 x^{3}}-\frac{152}{21 x^{4}}-\frac{968}{189 x^{5}}-\frac{632}{189 x^{6}} \\
& \ddot{b}_{r}^{\mathrm{q}}=\frac{1}{x}\left[\mathrm{~b}_{1}^{\mathrm{q}}+2 \ln (f)\right] \dot{b}_{r}^{\mathrm{q}}+\frac{1}{f}\left(\frac{29}{60 x}+\frac{2}{3 x^{2}}+\frac{2}{3 x^{3}}+\frac{8}{9 x^{4}}+\frac{4}{3 x^{5}}\right) \\
& b_{t}^{\circ}=\frac{1}{4} f\left(1-\frac{4}{3 x}\right) \\
& \dot{b}_{t}^{\circ}=\frac{1}{x}\left[\mathrm{~b}_{1}^{\circ}+2 \ln (f)\right] b_{t}^{\circ}+\frac{293}{280 x}-\frac{209}{84 x^{2}}+\frac{16}{35 x^{3}}+\frac{2}{3 x^{4}}+\frac{2}{9 x^{5}}+\frac{4}{45 x^{6}} \\
& \dot{b}_{r}^{\circ}=\frac{1}{f}\left(\frac{1}{20}-\frac{1}{12 x}\right) \\
& b_{t}^{\mathrm{h}}=\frac{1}{15} f\left(1-\frac{5}{2 x}+\frac{10}{7 x^{2}}\right)
\end{aligned}
$$

TABLE XV. Radial functions in $\left(t, r, \theta^{A}\right)$ coordinates: type- $\mathcal{P}$ and type- $\mathcal{Q}$ potentials. The functions are expressed in terms of $x:=r / M$ and $f:=1-2 / x$.

\begin{tabular}{ll}
\hline \hline$p_{t t}^{\mathrm{m}}=\frac{2}{x^{5}} \mathrm{p}^{\mathrm{m}}-f^{2}\left(\frac{1}{15}+\frac{2}{75 x}-\frac{4}{25 x^{2}}+\frac{32}{225 x^{3}}+\frac{32}{225 x^{4}}\right)$ & $q_{t t}^{\mathrm{m}}=\frac{2}{x^{5}} \mathrm{q}^{\mathrm{m}}+f^{3}\left(\frac{1}{15}+\frac{2}{25 x}+\frac{2}{25 x^{2}}+\frac{4}{75 x^{3}}\right)$ \\
$\left.p_{r r}^{\mathrm{m}}=\frac{2}{x^{5} f^{2}} \mathrm{p}^{\mathrm{m}}-\frac{1}{15}-\frac{2}{75 x}+\frac{4}{25 x^{2}}-\frac{32}{225 x^{3}}-\frac{32}{225 x^{4}}\right)$ & $q_{r r}^{\mathrm{m}}=\frac{2}{x^{5} f^{2}} \mathrm{q}^{\mathrm{m}}+\frac{1}{15}-\frac{4}{75 x}-\frac{2}{25 x^{2}}-\frac{8}{75 x^{3}}-\frac{8}{75 x^{4}}$ \\
$p^{\mathrm{m}}=\frac{2}{75}-\frac{8}{45 x^{2}}+\frac{128}{225 x^{5}}$ & $q^{\mathrm{m}}=\frac{2}{225}-\frac{2}{45 x}+\frac{32}{75 x^{5}}$ \\
$p_{t t}^{\mathrm{q}}=\frac{1}{x^{2}} \mathrm{p}^{\mathrm{q}} e_{t t}^{\mathrm{q}}-f^{2}\left(\frac{2}{7}+\frac{6}{7 x}-\frac{8}{7 x^{2}}\right)$ & $q_{t t}^{\mathrm{q}}=\frac{1}{x^{2}} \mathrm{q}^{\mathrm{q}} e_{t t}^{\mathrm{q}}+f\left(\frac{2}{7}-\frac{2}{3 x}\right)$ \\
$p_{r r}^{\mathrm{q}}=\frac{1}{x^{2}} \mathrm{p}^{\mathrm{q}} e_{r r}^{\mathrm{q}}-\frac{4}{7}-\frac{2}{7 x}+\frac{8}{7 x^{2}}$ & $q_{r r}^{\mathrm{q}}=\frac{1}{x^{2}} \mathrm{q}^{\mathrm{q}} e_{r r}^{\mathrm{q}}+\frac{1}{f}\left(\frac{4}{3}-\frac{18}{7 x}\right)$ \\
$p^{\mathrm{q}}=\frac{1}{x^{2}} \mathrm{p}^{\mathrm{q}} e^{\mathrm{q}}-\frac{1}{14}+\frac{12}{7 x^{4}}$ & $q^{\mathrm{q}}=\frac{1}{x^{2}} \mathrm{q}^{\mathrm{q}} e^{\mathrm{q}}+\frac{1}{2}-\frac{4}{7 x}$ \\
$p_{t t}^{\mathrm{h}}=\mathrm{p}^{\mathrm{h}} e_{t t}^{\mathrm{h}}+f^{2}\left(\frac{367}{36}-\frac{164}{9 x}+\frac{7}{x^{2}}\right)$ & \\
$p_{r r}^{\mathrm{h}}=\mathrm{p}^{\mathrm{h}} e_{r r}^{\mathrm{h}}+\frac{391}{36}-\frac{176}{9 x}+\frac{7}{x^{2}}$ & $q_{t t}^{\mathrm{h}}=\mathrm{q}^{\mathrm{h}} e_{t t}^{\mathrm{h}}-f\left(\frac{17}{36}-\frac{49}{54 x}\right)$ \\
$p^{\mathrm{h}}=\mathrm{p}^{\mathrm{h}} e^{\mathrm{h}}+\frac{391}{36}-\frac{868}{27 x}+\frac{206}{9 x^{2}}$ & $q_{r r}^{\mathrm{h}}=\mathrm{q}^{\mathrm{h}} e_{r r}^{\mathrm{h}}-\frac{1}{f}\left(\frac{7}{108}-\frac{1}{6 x}\right)$ \\
\hline \hline
\end{tabular}

TABLE XVI. Radial functions in $\left(t, r, \theta^{A}\right)$ coordinates: type- $\mathcal{G}$ and type- $\mathcal{H}$ potentials. The functions are expressed in terms of $x:=r / M$ and $f:=1-2 / x$.

\begin{tabular}{ll}
\hline \hline$g_{t t}^{\mathrm{d}}=0$ & \\
$g_{t r}^{\mathrm{d}}=f\left(\frac{2}{15}-\frac{8}{15 x}\right)$ & $h_{t}^{\mathrm{q}}=\frac{1}{x^{2}} \mathrm{~h}^{\mathrm{q}} b_{t}^{\mathrm{q}}+f\left(\frac{4}{21}+\frac{16}{21 x}+\frac{32}{21 x^{2}}\right)$ \\
$g_{r r}^{\mathrm{d}}=0$ & \\
$g^{\mathrm{d}}=0$ & \\
& \\
$g_{t t}^{\mathrm{o}}=\frac{1}{x} \mathrm{~g}^{\mathrm{o}} e_{t t}^{\circ}$ & \\
$g_{t r}^{\circ}=f\left(\frac{1}{9}-\frac{4}{9 x}\right)$ & $h_{t}^{\mathrm{h}}=\mathrm{h}^{\mathrm{h}} b_{t}^{\mathrm{h}}-f\left(\frac{8}{3 x}-\frac{64}{21 x^{2}}\right)$ \\
$g_{r r}^{\circ}=\frac{1}{x} \mathrm{~g}^{\mathrm{o}} e_{r r}^{\circ}$ & $h_{r}^{\mathrm{h}}=0$ \\
$g^{\circ}=\frac{1}{x} \mathrm{~g}^{\circ} e^{\circ}$ & \\
\hline \hline
\end{tabular}


[1] E. E. Flanagan and T. Hinderer, Constraining neutron star tidal Love numbers with gravitational wave detectors, Phys. Rev. D 77, 021502(R) (2008), arXiv:0709.1915.

[2] T. Hinderer, B. D. Lackey, R. N. Lang, and J. S. Read, Tidal deformability of neutron stars with realistic equations of state and their gravitational wave signatures in binary inspiral, Phys. Rev. D 81, 123016 (2010), arXiv:0911.3535.

[3] L. Baiotti, T. Damour, B. Giacomazzo, A. Nagar, and L. Rezzolla, Accurate numerical simulations of inspiralling binary neutron stars and their comparison with effective-one-body analytical models, Phys. Rev. D 84, 024017 (2011), arXiv:1103.3874.

[4] J. Vines, E. E. Flanagan, and T. Hinderer, Post-1-Newtonian tidal effects in the gravitational waveform from binary inspirals, Phys. Rev. D 83, 084051 (2011), arXiv:1101.1673.

[5] F. Pannarale, L. Rezzolla, F. Ohme, and J. S. Read, Will black hole-neutron star binary inspirals tell us about the neutron star equation of state?, Phys. Rev. D 84, 104017 (2011), arXiv:1103.3526.

[6] B. D. Lackey, K. Kyutoku, M. Shibata, P. R. Brady, and J. L. Friedman, Extracting equation of state parameters from black hole-neutron star mergers. I. Nonspinning black holes, Phys. Rev. D 85, 044061 (2012), arXiv:1109.3402.

[7] T. Damour, A. Nagar, and L. Villain, Measurability of the tidal polarizability of neutron stars in late-inspiral gravitationalwave signals, Phys. Rev. D 85, 123007 (2012), arXiv:1203.4352.

[8] J. S. Read, L. Baiotti, J. D. E. Creighton, J. L. Friedman, B. Giacomazzo, K. Kyutoku, C. Markakis, L. Rezzolla, M. Shibata, and K. Taniguchi, Matter effects on binary neutron star waveforms, Phys. Rev. D 88, 044042 (2013), arXiv:1306.4065.

[9] J. E. Vines and E. E. Flanagan, First-post-Newtonian quadrupole tidal interactions in binary systems, Phys. Rev. D 88, 024046 (2013), arXiv:1009.4919.

[10] A. Maselli, L. Gualtieri, and V. Ferrari, Constraining the equation of state of nuclear matter with gravitational wave observations: Tidal deformability and tidal disruption, Phys. Rev. D 88, 104040 (2013), arXiv:1310.5381.

[11] B. D. Lackey, K. Kyutoku, M. Shibata, P. R. Brady, and J. L. Friedman, Extracting equation of state parameters from black hole-neutron star mergers: Aligned-spin black holes and a preliminary waveform model, Phys. Rev. D 89, 043009 (2014), arXiv:1303.6298.

[12] M. Favata, Systematic parameter errors in inspiraling neutron star binaries, Phys. Rev. Lett. 112, 101101 (2014), arXiv: 1310.8288

[13] K. Yagi and N. Yunes, Love number can be hard to measure, Phys. Rev. D 89, 021303 (2014), arXiv:1310.8358.

[14] L. Wade, J. D. E. Creighton, E. Ochsner, B. D. Lackey, B. F. Farr, T. B. Littenberg, and V. Raymond, Systematic and statistical errors in a Bayesian approach to the estimation of the neutron-star equation of state using advanced gravitational wave detectors, Phys. Rev. D 89, 103012 (2014).

[15] S. Bernuzzi, A. Nagar, T. Dietrich, and T. Damour, Modeling the Dynamics of Tidally Interacting Binary Neutron Stars up to the Merger, Phys. Rev. Lett. 114, 161103 (2015), arXiv:1412.4553.

[16] B. D. Lackey, S. Bernuzzi, C. R. Galley, J. Meidam, and C. Van Den Broeck, Effective-one-body waveforms for binary neutron stars using surrogate models, Phys. Rev. D 95, 104036 (2017), arXiv:1610.04742.

[17] V. Ferrari, L. Gualtieri, and A. Maselli, Tidal interaction in compact binaries: A post-Newtonian affine framework, Phys. Rev. D 85, 044045 (2012), arXiv:1111.6607.

[18] A. Maselli, L. Gualtieri, F. Pannarale, and V. Ferrari, On the validity of the adiabatic approximation in compact binary inspirals, Phys. Rev. D 86, 044032 (2012), arXiv:1205.7006.

[19] A. Maselli, V. Cardoso, V. Ferrari, L. Gualtieri, and P. Pani, Equation-of-state-independent relations in neutron stars, Phys. Rev. D 88, 023007 (2013), arXiv:1304.2052.

[20] T. Hinderer, A. Taracchini, F. Foucart, A. Buonanno, J. Steinhoff, M. Duez, L. E. Kidder, H. P. Pfeiffer, M. A. Scheel, B. Szilagyi, K. Hotokezaka, K. Kyutoku, M. Shibata, and C. W. Carpenter, Effects of Neutron-Star Dynamic Tides on Gravitational Waveforms within the Effective-One-Body Approach, Phys. Rev. Lett. 116, 181101 (2016), arXiv:1602.00599.

[21] J. Steinhoff, T. Hinderer, A. Buonanno, and A. Taracchini, Dynamical tides in general relativity: Effective action and effective-one-body Hamiltonian, Phys. Rev. D 94, 104028 (2016), arXiv:1608.01907.

[22] W. Xu and D. Lai, Resonant tidal excitation of oscillation modes in merging binary neutron stars: Inertial-gravity modes, Phys. Rev. D 96, 083005 (2017), arXiv:1708.01839.

[23] T. Cullen, I. Harry, J. Read, and E. Flynn, Matter effects on LIGO/Virgo searches for gravitational waves from merging neutron stars, Class. Quantum Grav. 34, 245003 (2017), arXiv:1708.04359.

[24] I. Harry and T. Hinderer, Observing and measuring the neutron-star equation-of-state in spinning binary neutron star systems (2018), arXiv:1801.09972.

[25] B. P. Abbott et al., GW170817: Observation of Gravitational Waves from a Binary Neutron Star Inspiral, Phys. Rev. Lett. 119, 161101 (2017), arXiv:1710.05832.

[26] S. Taylor and E. Poisson, Nonrotating black hole in a post-Newtonian tidal environment, Phys. Rev. D 78, 084016 (2008), arXiv:0806.3052.

[27] T. Damour, M. Soffel, and C. Xu, General-relativistic celestial mechanics. I. Method and definition of reference systems, Phys. Rev. D 43, 3273 (1991).

[28] T. Damour, M. Soffel, and C. Xu, General-relativistic celestial mechanics. II. Translational equations of motion, Phys. Rev. D 45, 1017 (1992).

[29] T. Damour, M. Soffel, and C. Xu, General-relativistic celestial mechanics. III. Rotational equations of motion, Phys. Rev. D 47, 3124 (1993). 
[30] E. Poisson and I. Vlasov, Geometry and dynamics of a tidally deformed black hole, Phys. Rev. D 81, 024029 (2010), arXiv:0910.4311.

[31] N. K. Johnson-McDaniel, N. Yunes, W. Tichy, and B. J. Owen, Conformally curved binary black hole initial data including tidal deformations and outgoing radiation, Phys. Rev. D 80, 124039 (2009), arXiv:0907.0891.

[32] X.-H. Zhang, Multipole expansions of the general-relativistic gravitational field of the external universe, Phys. Rev. D 34, 991 (1986).

[33] K. Martel and E. Poisson, Gravitational perturbations of the Schwarzschild spacetime: A practical covariant and gaugeinvariant formalism, Phys. Rev. D 71, 104003 (2005), arXiv:gr-qc/0502028.

[34] E. Poisson and C. M. Will, Gravity: Newtonian, Post-Newtonian, Relativistic (Cambridge University Press, Cambridge, England, 2014).

[35] E. Racine and E. E. Flanagan, Post-1-Newtonian equations of motion for systems of arbitrarily structured bodies, Phys. Rev. D 71, 044010 (2005), arXiv:gr-qc/0404101. 